\title{
No where / now here
}

\author{
Citation for published version (APA):
}

Wilbur, K. (2021). No where / now here: context and competency expectations in workplace-based training. [Doctoral Thesis, Maastricht University]. ProefschriftMaken. https://doi.org/10.26481/dis.20210705kw

Document status and date:

Published: 01/01/2021

DOI:

$10.26481 /$ dis.20210705kw

Document Version:

Publisher's PDF, also known as Version of record

\section{Please check the document version of this publication:}

- A submitted manuscript is the version of the article upon submission and before peer-review. There can be important differences between the submitted version and the official published version of record.

People interested in the research are advised to contact the author for the final version of the publication, or visit the DOI to the publisher's website.

- The final author version and the galley proof are versions of the publication after peer review.

- The final published version features the final layout of the paper including the volume, issue and page numbers.

Link to publication

\footnotetext{
General rights rights.

- You may freely distribute the URL identifying the publication in the public portal. please follow below link for the End User Agreement:

www.umlib.nl/taverne-license

Take down policy

If you believe that this document breaches copyright please contact us at:

repository@maastrichtuniversity.nl

providing details and we will investigate your claim.
}

Copyright and moral rights for the publications made accessible in the public portal are retained by the authors and/or other copyright owners and it is a condition of accessing publications that users recognise and abide by the legal requirements associated with these

- Users may download and print one copy of any publication from the public portal for the purpose of private study or research.

- You may not further distribute the material or use it for any profit-making activity or commercial gain

If the publication is distributed under the terms of Article $25 \mathrm{fa}$ of the Dutch Copyright Act, indicated by the "Taverne" license above, 
NO WHERE | NOW HERE

Context and Competency Expectations in Workplace-Based Training 
This research reported here was carried out at

\section{Maastricht University}

In the School of Health Professions Education

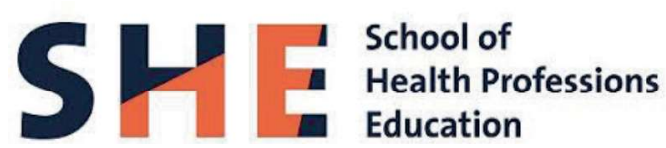

@Kerry Wilbur, Maastricht 2021

Cover Illustration by: Istockphoto || ProefschriftMaken

Printing: ProefschriftMaken || www.proefschriftmaken.nl

ISBN: 978-94-6423-304-9 


\section{NO WHERE I NOW HERE \\ Context and Competency Expectations in Workplace-Based Training}

ter verkrijging van de graad van doctor aan de Universiteit Maastricht, op gezag van de Rector Magnificus, Prof. dr. Rianne M. Letschert, volgens het besluit van het College van Decanen, in het openbaar te verdedigen op

maandag 5 juli 2021, om 16.00 uur

door

Kerry Wilbur 


\section{Supervisors}

Prof. dr. Erik W. Driessen

Prof. dr. Fedde Scheele

Prof. dr. Pim W. Teunissen

\section{Assessment Committee}

Prof. dr. Cees van der Vleuten (chair)

Prof. dr. Pierre Moreau, Kuwait University

Prof. dr. Miriam Oude Egbrink

Prof. dr. Michiel van Agtmael, VUMC Amsterdam

Dr. Dominique Waterval 


\section{Table of Contents}

Chapter 1

Introduction

\section{Chapter 2}

Describing student performance: a comparison among clinical preceptors across cultural contexts

Published in Medical Education

\section{Chapter 3}

Workplace-based assessment in cross-border health professional

education

Published in Teaching and Learning in Medicine

\section{Chapter 4}

Team member expectations of trainee communicator and collaborator competencies

Published in Medical Teacher

\section{Chapter 5}

Pharmacist trainees narrow scope of interprofessional collaboration and communication in hospital practice

Submitted

\section{Chapter 6}

Discussion

Summary

Samenvatting

Valorisation

Dissemination

She Dissertation Series

Acknowledgements 



\section{CHAPTER 1}

\section{Introduction}




\section{Introduction}

Pharmacists are recognized leaders in healthcare systems. The public continues to rank pharmacists among the most trusted professionals in national surveys conducted around the world year after year. ${ }^{1-3}$ Abilities to develop and maintain collaborative relationships with patients, knowledge to manage minor ailments or make appropriate referrals for further care and support, and participation in health and wellness promotion are just some of the roles instilling confidence in the public. While this favorable status is likely owed in large part to a collective of these accessible and positive patient-facing practices in community pharmacies, pharmacists are integral to patient medication management in other outpatient and inpatient care settings. In hospitals, pharmacists ensure safe and effective therapy reaches the diverse populations of admitted patients and contribute to the requisite decision-making on teams. ${ }^{4}$ Pharmacy programs are therefore responsible for equipping students with the professional competencies necessary to meet contemporary and emerging pharmacist scopes of practice in diverse care settings, for both current and future societal needs. Practice in authentic workplaces under supervision of clinical educators is inextricably linked to optimizing this training. Here, learners undertake patient care responsibilities assigned by supervisors who guide, but also judge these evolving abilities and ultimately readiness for independent professional practice. This thesis aims to enhance our understanding of workplace-based assessment and competency development in pharmacy education.

Competency-based education (CBE) in the health professions has been broadly defined as, "an outcomes-based approach to the design, implementation, assessment, and evaluation of medical education programs, using an organizing framework of competencies". ${ }^{5}$ This educational model is intended to ensure patients are ultimately cared for by qualified professionals who have met pre-defined graduate outcome abilities. ${ }^{6}$ Competency-based education has expanded beyond its origins for North American physician-training (hence a companion term, competency-based medical education (CBME)) to serve as an educational framework for multiple disciplines in diverse global jurisdictions. ${ }^{7,8}$ The adoption of CBE in pharmacy is similarly influenced by its contemporary application in medical education. ${ }^{9}$ In Canada, the conceptual shift for pharmacy programs to graduate "care providers who use their medication therapy expertise to benefit patients, communities and populations" emerged as a direct corollary to the antecedent CanMEDS educational framework whereby physicians are trained to be medical experts. ${ }^{10,11}$ The societal accountability ultimately underpinning this outcome-oriented training model aligns with pharmacists' fundamental mandate to provide patient-centred care to the individuals and communities they serve. ${ }^{12}$

Workplace-based learning is integral to the competency development of health professional trainees. ${ }^{13}$ Patient care environments are where learners have the opportunity to apply knowledge, practice skills, cultivate attitudes and behaviours - demonstrate the competencies - outlined by their respective professional programs. ${ }^{14,15}$ Effective workplacebased assessment (WBA) is therefore critical to a competency-based curriculum. ${ }^{16}$ Its processes and instruments ideally function to both record direct evidence of performance (assessment of learning) and stimulate feedback conversations with the trainee (assessment 
for learning). ${ }^{6,17,18}$ Since CBE is intended to represent the authentic work of health care providers, observation and subsequent judgement of trainees takes place in actual practice settings. For pharmacists, these training spaces comprise any context where medication management is required for patient care, including community pharmacies, primary care clinics, hospital pharmacies, and inpatient and outpatient wards. However, in contrast to competency assessment conducted under the relatively controlled testing conditions of objective structured clinical examinations (OSCE) or simulation-based training, WBA of health professional learners is rife with variability. ${ }^{19,20}$ The clinical learning environment is intrinsically unstandardized in countless and complex ways, involving the people and the place.

Competency-based based education in health professional programs rely on an array of practice-based partners to supervise trainees under diverse conditions of real professional work, guiding their learning and assessing performance. These clinical educators (known also as clinical teachers, supervisors, preceptors, or field instructors) are unequivocal assets to training programs across all health disciplines and specialties, yet quantitative evaluation of their trainee performance assessments produces troubling low inter-rater reliability. ${ }^{21,22}$ From a psychometric perspective, the collective understanding is that this undesirable variance lies not in "true score" variance (or in other words, inconsistencies in trainee performance) but instead arises from rater variance (e.g. measurement error among assessors). ${ }^{23}$ An explanatory paradigm in WBA follows that a true standard of competency performance exists to which assessors are unable to correctly follow or apply. ${ }^{24}$ To be clear, such deficits are not equated with supervisory negligence or malevolent abdications of responsibilities; on the contrary, human judgement is recognized as "fallible" and "imperfect". ${ }^{25}$ Informed by behavioural and psychological theories, we realize judgements assessors make of trainee performance in the clinical learning environment are subject to a host of influences (e.g. categorical thinking, stereotypes, schemas, unavoidable bias, contrast effects, cognitive workload) and a number of corresponding corrective strategies have been proposed. . $^{19,23,25-27}$

However, socio-cultural frameworks of clinical learning and assessment direct an alternate outlook regarding the idiosyncrasies of WBA. Student learning is a socially-mediated process within systems which also bear cultural, institutional, and historical attributes. ${ }^{15,28}$ Developing competencies for health professional practice are considered products of interactions with many individuals or groups across varied clinical learning environments and their interpretations may defy strict descriptions. ${ }^{29}$ The multeity of perspectives about student performances observed and assessed over time across places has high face validity. ${ }^{6,17}$ In a post-psychometric era, the collective is viewed, not as an error to be reconciled, but as yielding meaningful information - "many fallible judgements, summed together, create value".$^{30}$ If in this perspective we appreciate that competency development and demonstrated performance is also specific to these shifting contexts, we must concede that its assessment is as well. 
Pharmacist trainee competency development is embedded in the work conducted in diverse patient care and clinical learning environments worldwide. Bates and Ellaway have articulated six patterns by which these contexts can be explicitly characterized and encompass: 1) patient; 2) physical; 3) practice; 4) educational; 5) institutional; and 6) social elements. ${ }^{31}$ The latter two distinguishing features have also been framed as "healthcare" to broadly include the organizational values, practices, rules, and routines by site and "culture" to reflect the broader societal and cultural contexts in training communities. ${ }^{32}$ Contextual variability will influence competency concepts, for learners and assessors alike. Renditions, permutations, and amalgamations within and across these patterns culminate in the conditions of the clinical learning environment and shape supervisory expectations of trainee performance at that place and time. A sociocultural-oriented inquiry into workplace-based assessment can illuminate how competencies are internalized in dynamic patient care settings. Assessing competence is not simply about who is constructing the concept but also where - context influences both trainee performance and assessor outlook, and ultimately interpretations of what performance is deemed desirable.

Deliberate exploration of people (assessors) and place (context) in pharmacist competency development and assessment is salient, but largely neglected. Professional competenciesthe knowledge and skills necessary for patient care - are set through broad consultative and socially negotiated working groups of experts and stakeholders. ${ }^{33}$ In many health disciplines, they have been further organized into role-based competency frameworks. ${ }^{34}$ In turn, these frameworks guide instructional and assessment design in the respective curricula. As earlier described, contemporary national pharmacy training in Canada devised its educational outcomes using medicine as the central source reference - they were not developed de novo. If the genesis of any curriculum arises from the "aspirations or ideals that motivate the educational undertaking" adaptation and subsequent interpretation of such frameworks should be intentional. ${ }^{35}$ Despite apparent or pragmatic parallels, any incomplete assumptions of a mutual (or adjacent) professional and societal ethos between medicine and pharmacy or within pharmacy practice in distinct jurisdictions may be compounded if pharmacy complacently relies on the universality of physician-in-training data to understand how learners and assessors make meaning in their contexts. Such consideration is additionally relevant as Canada is perceived among the leaders in pharmacy practice and education globally and consequently other countries model their own pharmacist training

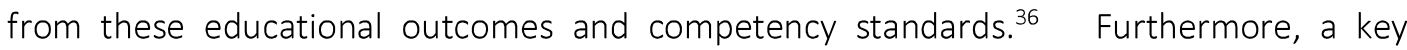
constituent to aspirational advanced pharmacy practice models includes purposeful expansion and integration within healthcare teams. ${ }^{37}$ Pharmacist learners and assessors must therefore also contend with superimposed interprofessional competency expectations of roles and work within the practice environment. Finally, the need for study of disciplinespecific workplace-based competency development and assessment is further underscored by available nascent study of pharmacist competency training revealing pharmacist faculty members view the requisite professional competency roles are best acquired by learners from clinical experience. ${ }^{38}$ 


\section{Research Questions}

The overall aim of this thesis is to enhance our understanding of workplace-based assessment and competency development in pharmacy education. Posed research questions include:

1. Can variations in clinical pharmacy supervisors' conceptualization of student performance be attributed to cross-cultural contexts?

2. In what ways do interprofessional team members expect pharmacist trainees to demonstrate competent collaboration and communication?

3. How do pharmacist trainees experience interprofessional collaboration and communication in the inpatient workplace-based setting?

\section{Research Setting and Context}

The studies described in this thesis were conducted at two major national pharmacy programs in the distinct international settings of Canada and Qatar. The backdrop to Chapters 2 and 3 was the State of Qatar, a small Arabian gulf emirate of (then) 2 million residents. Like other countries in the region who have experienced marked economic growth from oil and gas revenues, Qatar has devoted significant resources to augment provision of health services with parallel investments within the health education sector. As part of this enterprise, a number of branch campuses of various North American universities have been established to train dental hygienists, medical radiographers, nurses, paramedics, physicians, and respiratory therapists. ${ }^{39}$ While it is not a satellite college of another international affiliate-university as previously described, the College of Pharmacy (CPH) was formed and launched in the fall of 2007 at Qatar University (QU) as the first domestic pharmacist training program. ${ }^{40}$ Its curriculum (e.g. competency frameworks, teaching and assessment strategies) was devised to meet both local needs and Canadian-accreditation standards for pharmacy education. As part of the workplace-based curriculum, students participate in clerkships within the Hamad Medical Corporation network of varied inpatient care settings under the supervision of pharmacists, many of whom have also trained in a North American or Commonwealth pharmacist practice model. ${ }^{41}$

Chapters 4 and 5 are set in the inpatient workplace-based environments of the pharmacy program at the University of British Columbia (UBC). The Faculty of Pharmaceutical Sciences at UBC has the sole mandate to train people to be pharmacists in the province and enrols the largest undergraduate student body ( $\mathrm{N}=872$ ) of all eight English language programs in the country. As a Canadian-accredited program, it shares competency standards with the aforementioned Canadian-accredited program at QU CPH. ${ }^{36}$ In Chapter 4, we recruited multidisciplinary participants working at the largest teaching hospital and quaternary care site in British Columbia. Meanwhile, pharmacy student participants distributed across different small and large, teaching- and community-based inpatient sites throughout the province for their graduating (4th) year clerkships formed the study population in Chapter 5. 


\section{Reflexivity}

Subjectivity is often argued to be inherent to qualitative inquiry and warrants exercises in personal reflexivity- understanding ourselves in order to understand our orientations to the world and how these values and experiences influence research processes and intepretations. ${ }^{42}$ Several professional identities (past and present) converge to colour my outlook. I am a pharmacist who: trained in the model of our participants (student); supervised trainees in the manner under study (preceptor); worked in a mixed-disciplinary direct patient care setting (team member); and an educator and administrator possessing overall health professional program responsibilities and a researcher pursuing questions in the workplace-based curriculum. With cross-border movement during this dissertation work (ex-patriate to re-patriate), my ideas and perspectives are a bilateral diffusion of these experiences, mimicking my physical journey (West to East, and back again). Privileged concurrent personal movement through countless global jurisdictions has also influenced this personal reflexivity.

Throughout the doctoral research and learning processes, this worldview (understanding, positions, approaches) has continued to materialize and mature. A difficult (and likely incomplete) dispatch of the positivist traditions of a biomedical science background notwithstanding, I would now broadly identify with the constructivist stance. The research design was therefore influenced by this orientation and (in epistemological reflexive concession to lingering positivist persuasions) paired with quantitative data collection choices (Chapters 3 and 5) to expand meaning and enhance credibility. ${ }^{42}$

Monitoring and interrogation of my prevailing assumptions and attitudes, which invariably also shaped study data analysis, interpretation, representation, and dissemination, was aided throughout these years by regular meetings with supervisors, continuous analytic memo-ing, as well as critical dialogue (structured and unstructured alike) with those adjacent or outside the pharmacy and health professional education (fellow doctoral students, staff and visitors to UBC's Centre for Health Education Scholarship, other faculty and extradisciplinary university and international community members).

In fidelity to the constructivist paradigm, I acknowledge that interpretations of the world are socially constructed and yield "multiple realities" and therefore the reality presented and knowledge constructed in this dissertation is partial. ${ }^{43,44}$ 


\section{Thesis Outline}

No where - Now here explores practical issues associated with contextualization of professional competencies derived for pharmacy education. In the following chapters, we specifically address workplace-based assessment and competency development in the inpatient clinical learning environment through the study of four different perspectives: pharmacy preceptors in Qatar and in Canada (intraprofessional), multidisciplinary team members (interprofessional), and the pharmacy trainees themselves.

Chapter 2 explores how clinical pharmacy supervisors conceptualise student performance across different cross-cultural contexts. We report the dimensions of knowledge, skills, and attitudes by which pharmacists in Middle East countries and across Canada categorize trainee performance. We extend this examination of intraprofessional expectations within disparate geographic regions in Chapter 3. Using written student vignettes or composites of described workplace-based performance built from the first study findings, we systematically compare assessment characterizations made by clinical supervisors across Canada and in Qatar. In Chapter 4, we shift our focus beyond disciplinary expectations of pharmacy trainees' clinical work and behaviours in hospital practice. Through interview of team-members on an inpatient clinical training unit, we theorize how they characterize and judge communicator and collaborator competencies for trainees outside their own profession, using pharmacy students as the anchoring example. Chapter 5 reorients practice expectations in clinical learning environments to the learners themselves. We analyze longitudinal diary data recorded by pharmacy students during clerkships in their graduating year to ascertain their opportunities and expectations for communicator and collaborator competency development in hospital settings. Finally, in Chapter 6, these findings are synthesized into a broader discussion addressing the original research questions. Strengths and weaknesses of the dissertation are considered, as well as the implications for future research and practice. Given this thesis is based on studies published as separate journal articles over time, some repetition across the chapters will be found. 
Table 1. Dissertation Study Chapter Overview

\begin{tabular}{|c|c|c|c|c|}
\hline Chapter & Research Questions & Data Source & Analytic Approach & Setting \\
\hline Chapter 2 & $\begin{array}{c}\text { How do clinical } \\
\text { preceptors in pharmacy } \\
\text { conceptualise varying } \\
\text { levels of student } \\
\text { performance } \\
\text { Can contextual } \\
\text { differences that may exist } \\
\text { across different countries } \\
\text { be identified? }\end{array}$ & $\begin{array}{l}\text { semi- } \\
\text { structured } \\
\text { interviews }\end{array}$ & $\begin{array}{l}\text { content analysis } \\
\text { mapping } \\
\text { constructivist } \\
\text { grounded theory }\end{array}$ & $\begin{array}{l}\text { Middle East } \\
\text { (Qatar, } \\
\text { Saudi } \\
\text { Arabia, } \\
\text { Lebanon) } \\
\text { Canada }\end{array}$ \\
\hline Chapter 3 & $\begin{array}{c}\text { What are assessor } \\
\text { impressions of pharmacy } \\
\text { student workplace-based } \\
\text { performance in two } \\
\text { countries? } \\
\text { Are any variations in } \\
\text { assessor judgments } \\
\text { culturally informed? }\end{array}$ & $\begin{array}{c}\text { student } \\
\text { vignettes and } \\
\text { semi- } \\
\text { structured } \\
\text { interviews }\end{array}$ & $\begin{array}{l}\text { modified Delphi } \\
\text { consensus } \\
\text { thematic analysis }\end{array}$ & $\begin{array}{l}\text { Qatar } \\
\text { Canada }\end{array}$ \\
\hline Chapter 4 & $\begin{array}{c}\text { How do interprofessional } \\
\text { team members expect } \\
\text { pharmacist trainees to } \\
\text { demonstrate competent } \\
\text { collaboration and } \\
\text { communication? } \\
\text { What are their } \\
\text { experiences offering } \\
\text { feedback about } \\
\text { pharmacist trainees? }\end{array}$ & $\begin{array}{c}\text { semi- } \\
\text { structured } \\
\text { interviews }\end{array}$ & $\begin{array}{c}\text { constructivist } \\
\text { grounded theory }\end{array}$ & Canada \\
\hline Chapter 5 & $\begin{array}{l}\text { How do pharmacist } \\
\text { trainees experience } \\
\text { interprofessional } \\
\text { collaboration and } \\
\text { communication in the } \\
\text { inpatient workplace- } \\
\text { based setting? }\end{array}$ & $\begin{array}{l}\text { longitudinal } \\
\text { diary records } \\
\text { and semi- } \\
\text { structured } \\
\text { interviews }\end{array}$ & $\begin{array}{l}\text { content analysis } \\
\text { mapping } \\
\text { reflexive thematic } \\
\text { analysis }\end{array}$ & Canada \\
\hline
\end{tabular}




\section{References}

1. Blank C. Pharmacists Top Most Trusted Professions in Gallup Poll. https://www.drugtopics.com/view/pharmacists-top-most-trusted-professions-galluppoll. Published 2020. Updated January 10, 2020. Accessed October 11, 2020.

2. Lynas K. Professionals you can trust: pharmacists top the list again in Ipsos Reid survey. Canadian Pharmacists Journal: Can Pharm J. 2012;145:55.

3. Worldwide ranking: trust in professions. GfK Compact. GfK Verein Study, Trust in Professions Web site. https://www.nim.org/en/compact/focustopics/worldwideranking-trust-professions. Published 2016. Accessed October 11, 2020.

4. Kaboli PJ, Hoth AB, McClimon BJ, Schnipper JL. Clinical pharmacists and inpatient medical care: a systematic review. Arch Intern Med. 2006;166:955-964.

5. Frank JR, Snell LS, Cate OT, et al. Competency-based medical education: theory to practice. Med Teach. 2010;32:638-645.

6. Lockyer J, Carraccio C, Chan M-K, et al. Core principles of assessment in competencybased medical education. Med Teach. 2017;39:609-616.

7. Frenk J, Chen L, Bhutta ZA, et al. Health professionals for a new century: transforming education to strengthen health systems in an interdependent world. The Lancet. 2010;376:1923-1958.

8. Frank JR, Snell L, Englander R, Holmboe ES. Implementing competency-based medical education: Moving forward. Med Teach. 2017;39:568-573.

9. Katoue MG, Schwinghammer TL. Competency-based education in pharmacy: A review of its development, applications, and challenges. J Eval Clin Pract. 2020;26:1114-1123.

10. Association of Faculties of Pharmacy in Canada (AFPC). Educational Outcomes for First Professionals Degree Programs in Pharmacy in Canada 2017. Ottawa, ON; 2017.

11. Warken M, Gordon J, Campbell C, Aboulsoud S. Developing and piloting national CPD accreditation standards: State of Qatar. Paper presented at: AMEE 2015; Glasgow, Scotland.

12. Dolovich L, Austin Z, Waite N, et al. Pharmacy in the 21st century: Enhancing the impact of the profession of pharmacy on people's lives in the context of health care trends, evidence and policies. Can Pharm J. 2018;152:45-53.

13. Manley K, Titchen A, Hardy S. Work-based learning in the context of contemporary health care education and practice: A concept analysis. Pract Dev Health Care. 2009;8:87-127.

14. Englander R, Cameron T, Ballard AJ, Dodge J, Bull J, Aschenbrener CA. Toward a common taxonomy of competency domains for the health professions and competencies for physicians. Acad Med. 2013;88:1088-1094. 
15. Yardley S, Teunissen PW, Dornan T. Experiential learning: AMEE guide No. 63. Med Teach. 2012;34:e102-e115.

16. Norcini J, Burch V. Workplace-based assessment as an educational tool: AMEE Guide No. 31. Med Teach. 2007;29:855-871.

17. Harris $\mathrm{P}$, Bhanji F, Topps $\mathrm{M}$, et al. Evolving concepts of assessment in a competencybased world. Med Teach. 2017;39:603-608.

18. Driessen $E$, Scheele F. What is wrong with assessment in postgraduate training? Lessons from clinical practice and educational research. Med Teach. 2013;35:569-574.

19. Govaerts M, van der Vleuten CP. Validity in work-based assessment: expanding our horizons. Med Educ. 2013;47:1164-1174.

20. Schuwirth L, Ash J. Assessing tomorrow's learners: In competency-based education only a radically different holistic method of assessment will work. Six things we could forget. Med Teach. 2013;35:555-559.

21. Sutkin G, Wagner E, Harris I, Schiffer R. What makes a good clinical teacher in medicine? A review of the literature. Acad Med. 2008;83:452-466.

22. Schauber SK, Hecht M, Nouns ZM. Why assessment in medical education needs a solid foundation in modern test theory. Adv Health Sci Educ Theory Pract. 2018;23:217-232.

23. Gingerich A, Regehr G, Eva KW. Rater-based assessments as social judgments: rethinking the etiology of rater errors. Acad Med. 2011;86:S1-S7.

24. Govaerts MJ, Van der Vleuten CP, Schuwirth LW, Muijtjens AM. Broadening perspectives on clinical performance assessment: rethinking the nature of in-training assessment. Adv Health Sci Educ Theory Pract. 2007;12:239-260.

25. Gingerich A, Kogan J, Yeates P, Govaerts M, Holmboe E. Seeing the 'black box' differently: assessor cognition from three research perspectives. Med Educ. 2014;48:1055-1068.

26. Yeates $P, O$ 'Neill P, Mann K, Eva K. Seeing the same thing differently. Mechanims that contribute to assessor differences in directly-observed performance assessments. Adv Health Sci Educ Theory Pract. 2013;18:325-341.

27. Gauthier G, St-Onge C, Tavares W. Rater cognition: review and integration of research findings. Med Educ. 2016;50:511-522.

28. Gipps C. Chapter 10: Socio-cultural aspects of assessment. Rev Res Educ. 1999;24:355392.

29. Crossley J, Jolly B. Making sense of work-based assessment: Ask the right questions, in the right way, about the right things, of the right people. Med Educ. 2012;46:28-37.

30. Hodges B. Assessment in the post-psychometric era: learning to love the subjective and collective. Med Teach. 2013;35:564-568. 
31. Bates J, Ellaway RH. Mapping the dark matter of context: a conceptual scoping review. Med Educ. 2016;50:807-816.

32. Schrewe B, Ellaway RH, Watling C, Bates J. The contextual curriculum: Learning in the matrix, learning from the matrix. Acad Med. 2018;93:1645-1651.

33. Frank JR, Mungroo R, Ahmad Y, Wang M, De Rossi S, Horsley T. Toward a definition of competency-based education in medicine: a systematic review of published definitions. Med Teach. 2010;32:631-637.

34. Tong B, St John M, Li E, Wilbur K. Could interprofessional education assessment in workplace learning be streamlined? J Interprof Educ Pract. 2020;19:100321.

35. McCowan T. Curricular transposition in citizenship education. Theory Res Educ. 2008;6:153-172.

36. The Canadian Council for Accreditation of Pharmacy Programs. Accreditation standards for the first professional degree in pharmacy programs. Toronto, ON; 2014.

37. Grymonpre R, Paradis E. Are pharmacy students learning to be effective collaborators and to work within health care teams through our interprofessional education initiatives? Can J Hosp Pharm. 2016;69:420-424.

38. Paradis E, Zhao R, Kellar J, Thompson A. How are competency frameworks perceived and taught? Perspect Med Educ. 2018;7:200-206.

39. El-Awaisi A, Wilby KJ, Wilbur K, El Hajj MS, Awaisu A, Paravattil B. A Middle Eastern journey of integrating Interprofessional Education into the healthcare curriculum: a SWOC analysis. BMC Med Educ. 2017;17:15.

40. Kheir N, Zaidan M, Younes H, El Hajj M, Wilbur K, Jewesson PJ. Pharmacy education and practice in 13 Middle Eastern countries. Am J Pharm Educ. 2008;72:133.

41. Wilbur K, Paiva M, Black EK. Pharmacy Student and preceptor impressions of faculty liaison visits to experiential training sites. Am J Pharm Edu. 2015;79:134.

42. Berger R. Now I see it, now I don't: Researcher's position and reflexivity in qualitative research. Qual Res. 2015;15:219-234.

43. Durdella N. Framing a dissertation study through a research tradition. In: Durdella N, ed. Qualitative dissertation methodology: A guide for research design and methods. Thousand Oaks, CA: Sage Publications; 2017:87-142.

44. Denzin NK, Lincoln YS. The Sage handbook of qualitative research. Thousand Oaks, CA: Sage Publications; 2011.

45. Kivunja $C$, Kuyini $A B$. Understanding and applying research paradigms in educational contexts. Int J High Educ. 2017;6:26-41. 



\section{CHAPTER 2}

\section{Describing student performance: a comparison among clinical preceptors across cultural contexts}

Published as: Wilbur K, Hassaballa N, Mahmood OS, Black EK. Describing student performance: a comparison among clinical preceptors across cultural contexts. Med Educ 2017;51:411-422.

DOI: $10.1111 /$ medu. 13223 


\begin{abstract}

\section{Introduction}

Health professional student evaluation during experiential training is notably subjective and assessor judgments may be affected by socio-cultural influences. We sought to explore how clinical preceptors in pharmacy conceptualize varying levels of student performance and what contextual differences may exist across different countries.
\end{abstract}

\title{
Methods
}

The qualitative research design employed semi-structured interviews. A sample of twenty clinical preceptors for post-baccalaureate Doctor of Pharmacy programs in Canada and the Middle East gave personal accounts of students they supervised who fell below, met, or exceeded their expectations. Discussions were analyzed following constructivist grounded theory principles.

\section{Results}

Seven major themes encompassing how clinical pharmacy preceptors categorize levels of student performance and behaviour were identified: knowledge; team interaction; motivation; skills; patient care; communication; and professionalism. Expectations were outlined using both positive and negative descriptions. Pharmacists typically described supervisory experiences representing a series of these categories, but arrived at concluding judgments in a holistic fashion; if valued traits of motivation and positive attitude were present, overall favourable impressions of a student could be maintained despite few observed deficiencies. Some prioritized dimensions could not be mapped to defined existing educational outcomes. We found no difference in thresholds for how student performance was distinguished by participants in the two regions.

\section{Conclusions}

Our research findings are congruent with current literature related to the subjectivity of health professional student assessment by clinical supervisors during experiential training and the first to grant insight into cross-national perspectives in pharmacy. As previously determined in social work and medicine, study of how evaluation instruments and associated processes can integrate these judgements should also be pursued in this discipline. 


\section{Introduction}

The global health care education community is becoming increasingly interconnected as universities with health faculties of repute establish affiliate or satellite training programmes abroad. This is but one example of ways in which health professional curricula and faculty staff are transplanted in cross-border partnerships. Commonly, educational brands (typically of Western origin) introduce branch campuses in foreign settings predominantly staffed by 'home' faculty members or, alternatively, Western study plans are adopted by domestic institutions but largely delivered by local faculty staff..$^{1-3}$ Host countries thereby facilitate access to high-calibre training for place-bound students and expand their skilled local workforce capacity, while esteemed source programmes further extend their reach in health professional education expertise, often under lucrative agreements. ${ }^{1}$ Although such academic relationships seem mutually beneficial, problems can arise from the conflict inherent in the delivery of identical, yet locally adapted, curricula, as demonstrated in studies of the cultural effects on problem-based learning and communication training..$^{4-8}$ Similar issues may also be anticipated to impact aspects of the intended workplace-based curriculum.

Within such curricula, health professional training programmes worldwide rely heavily on clinicians ('clinical preceptors', 'supervisors' or 'field instructors') to support student learning and development by delegating patient care tasks, and providing associated mentored problem solving, case-based discussion and formative feedback. Additionally, these clinicians' judgements of practice-based performance are used to inform programme decisions related to student advancement through the curriculum and ultimately suitability for graduation. In this regard, most health disciplines employ evaluation tools for use by clinicians in the assessment of student abilities in these patient care settings. Many of these instruments use quantitative measures that compel preceptors to select a numeric rating from a scale that may then culminate with an overall rotation grade, and include space earmarked for additional written comments. ${ }^{9,10}$ Other evaluation tools are developed to generate a score arising from a checklist of specific behaviours, such as the mini-clinical examination (mini-CEX), objective structured clinical examination (OSCE) and other formats for the direct observation of performance in the clinical setting. ${ }^{11}$

However, problems with the reliability and validity of the findings from such workplacebased assessment (WBA) strategies have been well described. Quantitative rating scales do not ensure standardisation as preceptors do not necessarily share understanding of the numeric response format or the performance descriptors that are often added to evaluation tools. ${ }^{12-14}$ For example, researchers have described how clinical faculty staff scoring of the same videotaped residents varied significantly on a 9-point Likert scale, ranging from 'unsatisfactory' to 'superior'. ${ }^{15}$ Think-aloud exercises in similar studies discover raters actually often agree upon the observed performance, but prioritise different aspects when characterising student abilities and subsequently assigning grades. ${ }^{16,17}$ Even when explanatory written narrative infrequently accompanies submitted evaluations, uniformity in their interpretation is lacking. ${ }^{14,18-20}$ Preceptors may employ language that, intentionally or not, serves to cue others to rank student performance, but not necessarily in a consistent fashion. ${ }^{21,22}$ Furthermore, the language preceptors choose to express valued student 
attributes does not always fit the framework of many competency-based assessment forms. ${ }^{23-26}$

Reconciling these issues of variability in rater perceptions and cultural influences on such perceptions demands consideration of the cultural context in which assessment takes place. Just as experiential training may be viewed through the lenses of social-cultural theories of learning, so too is its assessment in that student performance is interpreted according to the perceptions and values of the evaluator. ${ }^{16,26}$ Although variability in preceptor WBA is recognised as being in part attributable to context-specific student performance, these views often refer to situational, not necessarily cultural, contexts. ${ }^{27}$ To date, investigations of how preceptors form impressions, and make, express and interpret judgements in WBA have been conducted in Western settings. In the face of continued internationalisation of health professional education, caution must be exercised when these transplanted curricula also export assessment instruments and implicit expectations for use in new environments. Existing evaluation practices and beliefs related to how clinical work is undertaken are among the local factors that must be purposefully considered. ${ }^{28-30}$ Just as questionnaires require adaptation when the source language or translated document is used in another country, review of the appropriateness of intended assessment processes overseas is necessary. ${ }^{31}$

In a study of WBA within a transplanted pharmacy education curriculum, we sought to explore how clinical preceptors conceptualise varying levels of student performance. We hypothesised that differences in assessment criteria exist among pharmacists contributing to experiential training in similar programmes in different cultural contexts, namely the Middle East and Canada.

\section{Methods}

\section{Programme Description}

The Doctor of Pharmacy (PharmD) programme in Canada was first devised as a graduate programme for those who had completed the baccalaureate degree necessary to enter practice as a pharmacist. PharmD programmes support the training of students to assume positions in advanced pharmacy practice models of care in which pharmacists are integrated members of multidisciplinary teams and collaborate with other clinicians in evidence-based patient management. ${ }^{32}$ When the first pharmacist training programme in the Middle East country of Qatar was developed, it adopted the Canadian curricular model and was subsequently the first overseas programme to be accredited by the Canadian Council for Accreditation of Pharmacy Programs (CCAPP). These accreditation standards outline the professional and educational attributes deemed as essential for pharmacy programmes intending to develop students who are patient-focused clinicians. The PharmD curriculum at Qatar University (QU) is currently a 36-credit post-baccalaureate programme that includes 32 weeks of experiential training (in eight different advanced clinical internships) with pharmacist mentors in patient care settings in Qatar. ${ }^{33}$ The Bachelor of Pharmacy degree has been widely unavailable in the USA for over 20 years as the entry-to-practice qualification converted to the PharmD degree, now obtained after at least 6 years of professional study. 
This transition to the PharmD degree as the entry-to-practice qualification is now underway at Canadian faculties of pharmacy. ${ }^{34}$

\section{Sample Population}

We explored perceptions of post-baccalaureate PharmD student performance formed by clinical preceptors through semi-structured interviews. Pharmacists were required to have supervised at least two post-baccalaureate PharmD student rotations to be considered for inclusion. At the time of our study, 40 local clinical pharmacists were serving as advanced clinical internship preceptors in the QU College of Pharmacy PharmD programme. Five of 24 eligible preceptors were randomly selected and invited to participate. We concurrently sought the views of pharmacists precepting post-baccalaureate PharmD students enrolled in accredited programmes in Canada, as well as elsewhere in the Middle East region (Lebanon and Saudi Arabia). Finally, we interviewed all North American-trained crossappointed pharmacy faculty members supervising PharmD students in clinical practice in Qatar ( $n=5$ at the time of the study). Two of these had also previously precepted students in North American settings. Overseas preceptors were purposively sampled and invited by e-mail to participate. The number of programmes in each country with eligible subjects and the dynamic nature of preceptor participation from year to year meant that accurate numbers of clinical supervisors in the population were not readily available to the researchers. The sampling strategy and participant numbers were based on prior published experience and the expectation that data saturation would be reached with the proposed sample size. ${ }^{35,36}$

\section{Participant Interviews}

Two trained researchers conducted 20-30-minute interviews with participants who offered informed consent. A pilot interview was first conducted to evaluate the proposed script, rehearse anticipated probes, and arrive at a realistic time estimate to share with potential subjects. Discussion between interviewer and subject ensued from the same three openended questions. Preceptors were asked to recall their own experiences in supervising PharmD students and to describe specific situations in which they had felt the student: (i) met expectations; (ii) exceeded expectations, and (iii) fell below expectations. Researchers asked for the three descriptions one at a time and varied the sequence (by, for example, not always starting the interview seeking descriptions of how a student had fallen below the preceptor's expectations). Participants were free to offer descriptions about any aspect of student performance or behaviour. However, if the preceptor instead described general traits related to these expectations, the researchers prompted the preceptor to think of a particular student in order to outline a specific example or 'story'. To ensure confidentiality, researchers asked participants not to mention student names. Additional probes were used to elicit comparisons. For example, if a preceptor described a situation in which the student had exceeded expectations, the researcher would ask how this might contrast with a student who, in the preceptor's view, had met expectations. 
Analysis

The discussions were audio-recorded and transcribed verbatim. One researcher attending the interview generated an initial transcription that was subsequently verified for accuracy and completeness by a second researcher. Data analysis began in parallel with ongoing interviews and alternated among the four different participant groups to ensure opportunities to examine any areas in more detail before data collection concluded.

We adopted a grounded theory methodology rooted in the constructivist theoretical perspective and employed corresponding approaches for coding, memo writing, sorting, negative case analysis, and constant comparison. ${ }^{37}$ Constructivism assumes a relativist position which excludes an absolute truth, but instead would consider student supervision as a subjective experience to be explored and understood as represented by the preceptor's reflection on said experiences. ${ }^{38}$ Three authors read the transcripts in an open coding process in which they labelled topics considered of interest and met repeatedly as a group to refine coding as more interviews were completed. Agreements were made through consensus and discussions continued until a stable coding structure had been created. Through constant comparison and review of researcher memos documented throughout the process, categories were further defined (by merging and deletion) and relationships established. We then put forth a series of concepts generated from these data, and relationships within and across participant groups.

Finally, as a quantitative means to further explore responses among the four participant groups, we determined key words related to the devised final themes and conducted a search of all interview texts to determine the counts and relative frequencies of these words. Ethics approval was obtained from the institutional review board of QU.

\section{Results}

A total of 20 interviews were conducted, including five with participants from each category: local staff in Qatar; regional staff (Lebanon, Saudi Arabia); staff in Canada, and North American-trained faculty staff in Qatar. Four of the native Middle Eastern subjects had undertaken part of their student training in North America. Demographic data for the participating clinical pharmacist preceptors are described in Table 1. It was felt that theoretical saturation was reached by the 17th interview, but we enrolled three additional participants (one from Qatar and two from Saudi Arabia) to confirm that no new data would emerge from subsequent analysis. 
Table 1. Clinical Pharmacy Preceptor Characteristics

\begin{tabular}{|l|l|l|l|l|l|}
\hline CHARACTERISTIC & Overall & Qatar & $\begin{array}{l}\text { Middle } \\
\text { East } \\
\text { Region }\end{array}$ & Canada & $\begin{array}{l}\text { Qatar } \\
\text { Faculty }\end{array}$ \\
& $\mathrm{N}=20(\%)$ & $\mathrm{N}=5(\%)$ & $\mathrm{N}=5(\%)$ & $\mathrm{N}=5(\%)$ & $\mathrm{N}=5(\%)$ \\
\hline Female & $8(40)$ & $3(75)$ & $1(10)$ & $2(40)$ & $2(40)$ \\
\hline Precepting experience, years* & $7.6 \pm 3.8$ & $5.6 \pm 2.7$ & $6.7 \pm 3.0$ & $13.2 \pm 2.5$ & $5.0 \pm 3.0$ \\
\hline PharmD students precepted, $\mathrm{n}(\%)$ & $11(55)$ & $4(80)$ & $0(0)$ & $2(40)$ & $5(100)$ \\
$<25$ & $4(20)$ & $1(20)$ & $3(67)$ & $1(20)$ & $0(0)$ \\
$25-75$ & $5(25)$ & $0(0)$ & $2(33)$ & $2(40)$ & $0(0)$ \\
$>75$ &
\end{tabular}

* mean and standard deviation

From these interviews, seven major themes were determined to represent how clinical preceptors described PharmD student performance (Table 2).

Table 2. Major categories arising from interviews with PharmD preceptors discussing graduate students meeting, exceeding or falling below expectations

\section{Major Category}

KNOWLEDGE

Knowledge base

Knowledge translation

\section{Definition}

Demonstrates ability to recall disease processes and therapeutic knowledge (including but not limited to, treatment alternatives, doses, mechanisms of action, anticipated side effects)

Applies information and knowledge to specific patient- cases Retains learned information from earlier in the rotation for future use in care

\section{TEAM INTERACTION}

Integration

Swiftly adapts to the care setting and work environment; exhibits good judgment in timing recommendations and is accepted by the team

Contribution

Contributes to direct patient care, including evidence-based patient decision-making discussions at bedside rounds; decisions are based on good judgment; does not avoid responsibility for care

\section{MOTIVATION}

Independence
Proactively approaches work (patient care, assignments) with minimal prompting; demonstrates advance and on-going selfstudy/preparation; anticipates tasks and often provides extra efforts in assigned tasks 
Disposition

Exhibits enthusiasm, and curiosity in patient care and the learning process; preceptor and team members enjoy working with and teaching them

SKILLS

Critical thinking

Demonstrates systematic process in patient-care and decision-making; can adapt accordingly when new information is provided; judgments are appropriate and safe

Information management

Efficiently retrieves and accurately appraises literature associated with drug therapy and patient management for their own learning and others' learning and decision-making purposes

\author{
Organization
}

Handles and prioritizes multiple tasks; punctual with assignments

\title{
PATIENT CARE
}

Interacts comfortably with patients; caring attitude; seeks to inform and advocates improved patient management

\section{COMMUNICATION}

Presentation/Written

Health care members
Creative and informative means to share information (presentation); clearly and succinctly summarizes information (presentation/chart note/written assignments)

Can comfortably and appropriately engage in polite disagreements related to patient-care and decision-making; shares information with preceptor and other health professionals in clear and succinct fashion

\section{PROFESSIONALISM}

Conduct

Respects preceptor expertise and time; demonstrates commitment to the rotation (arrives early and leaves late); appreciates learning opportunities

Insight
Reflects and responds to constructive feedback; never overconfident; recognizes limitations

These categories included student knowledge, team interaction, motivation, skills, patient care, communication, and professionalism. Individual descriptions of these points could be discussed in either positive or negative perspectives. For example, one preceptor may point out inability to adequately manage a reasonable case load as an indicator of organizational deficiencies in their experience with a student falling below his or her expectations and another may use the perceived systematic processes in the approach to care of multiple patients to outline a former student who exceeded his or her expectations. No individual preceptor interview covered all aspects of performance or behavior that were ultimately compiled into the final themes, but we found consistent features within student categories. Students who exceeded expectations were commonly described as those who were committed and proactive in their internship tasks. 
"I think the most important point for this [excellent] student was that she was not just waiting for me to give the answers. She was trying to find more questions and answers to start discussions with me. She also tried to improve the standards of care." [Preceptor D in Qatar]

"The [average[ student was more reactive in her interactions with the team. She was respectful and responded to their questions, but wouldn't go out and search out more." [Preceptor A in Canada]

"A [weak] student I had was very disinterested in rounds. While the medical students were discussing papers, this student was simply sitting there or looking at the phone...or staring out the window into the sky! It is simply...they give you the impression they are not interested. They show it and they show everybody else. I mean, it is not a good image to the medical team in an interdisciplinary round. The physician or the nurse, they are not going to trust what you say." [Preceptor C in KSA]

"I still remember one of my students, she told me she slept 2 hours because of the assignments I gave her. So this doesn't make me happy and I felt little sad about it, but the same time I am very happy about her eagerness and about her desire for knowledge. Such students, they even push the preceptor high and they make us gain up-to-date knowledge." [Preceptor E in Qatar]

Preceptors pointed out aspects of safe and appropriate decision-making when referring to certain students in terms of being a peer or "colleague".

"So, in the sense I think the ideal student is the one closest already functioning somewhat like a pharmacist. Yes they are going have some limitations in their knowledge base, but they realize their limitations." [Preceptor B in Canada]

"We want [an excellent student] to be our colleagues and we will be proud to have them. And when one of them applied, for example, to the position in our hospital all of the preceptors went to supervisor's office and gave great recommendations "This student just applied to pharmacist position, and I would recommend you to hire her and you wouldn't find a better alternative".

[Preceptor D in KSA]

The continuum between what preceptors identified in their experiences as a student who met and conversely fell below his or her expectations did not differ between our subject groups. Canadians, North Americans in the Middle East, and Arab preceptors represented those with poor knowledge requiring constant coaching coupled with inability to complete tasks reliably as students they would not pass.

"A patient is taking digoxin and I ask, 'ok, what are the drug interactions with digoxin?'. [The student has] no idea. So I ask him to look them up. Next day, I ask, 'ok, what are the drug interactions with digoxin?'. [The student responds], 'Oh, um...'. I tell him, 'ok, you need to look that up'. The third day, the patient is started on a drug that has an interaction with digoxin. Then I was, "I warned you this was going to happen, you let the physician order this drug that has a major interaction.' And still, he was, 'oh really? I don't think there is'! The average student would have gone and looked up all them. The exceptional student would have been able to list them off immediately and as soon as the order was written, would have been able to intervene." [Preceptor C in Canada] 
Irrespective of region, all preceptors described students in a holistic fashion. Many overtly identified that if a student responded to feedback and made discernable efforts, the clinical preceptor was likely to overlook almost any type of observed deficiencies (not jeopardizing patient safety) and categorized overall performance as meeting expectation.

"We are humans and everyone might have his personal complications; however, we can help each other and solve the problems. Actually, I had an example of a PharmD student - she was excellent. During the rotation, she had some personal issues and I really appreciated her contribution, although it was not $100 \%$, but she was trying her best to reach a high level and I was happy to give her the maximum grade because of that." [Preceptor A in Qatar]

The frequencies at which key words related to each category appeared across all interviews are illustrated in Fig. 1. Collectively, the categories referred to most frequently concerned the student's patient care abilities, knowledge base, and communication with physicians and health care team members. When the various student features addressed among the preceptor groups were ranked, few differences arose. For example, the Arab clinical preceptors interviewed were more likely to point out aspects of student conduct, such as appropriate dress and demonstrations of respect, whereas the North American-trained faculty members who supervise students in Qatar were the only preceptors to prioritise critical thinking skills.

\section{Figure 1. Frequency of keywords related to themes arising from a grounded theory analysis of interview transcripts of clinical preceptors across regions}

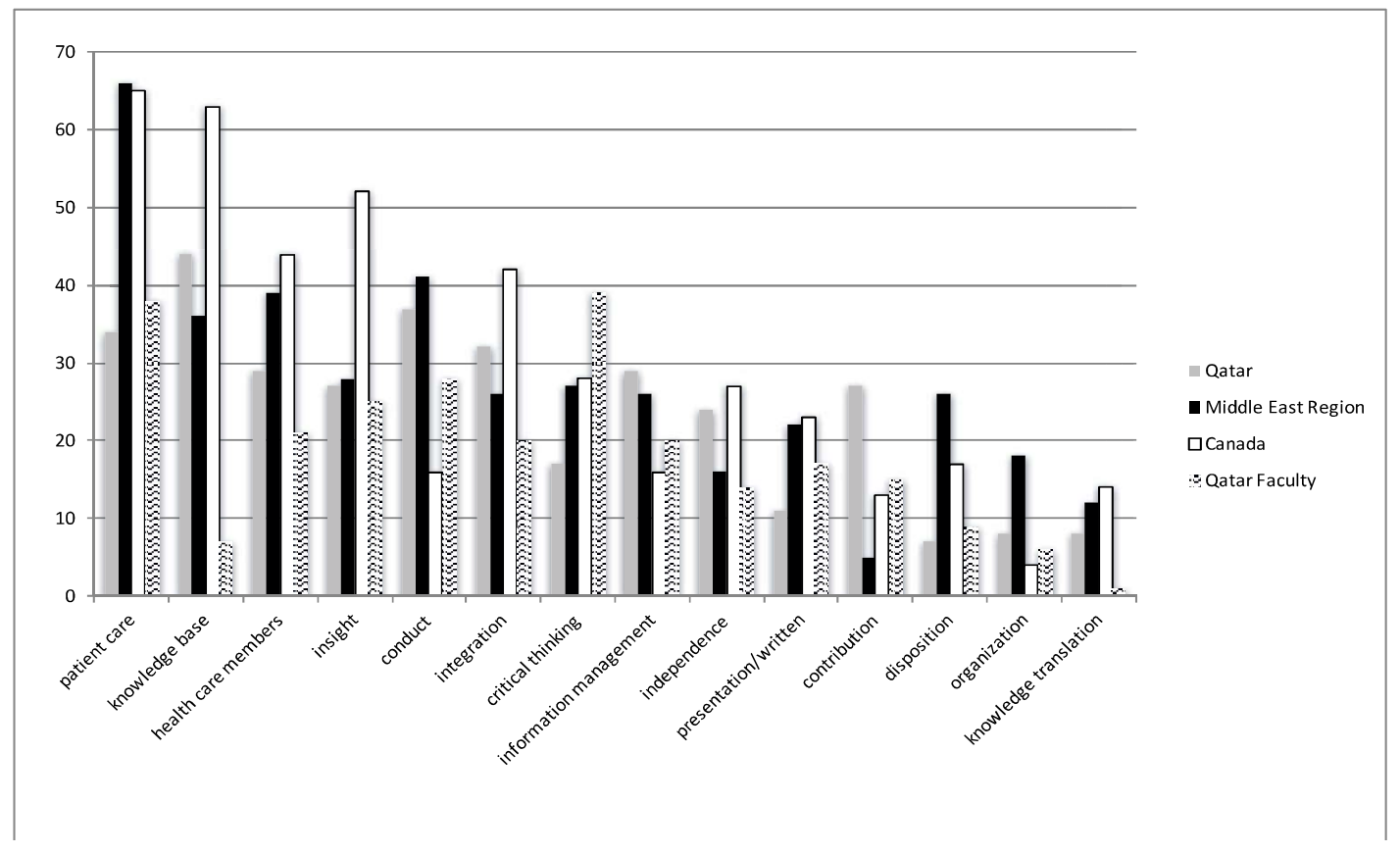


Using the Association for Faculties of Pharmacy in Canada (AFPC) educational outcomes for pharmacy degree programmes in Canada, we subsequently mapped the categories that emerged from our study interviews to the educational outcomes outlined in both the postbaccalaureate and the entry-to-practice pharmacy programme documents (Appendix S1). ${ }^{39,40}$ The terminology in these AFPC outcomes is modelled after that used in the CanMEDS format for medicine in Canada. ${ }^{41}$

\section{Appendix S1}

Educational Outcomes for a Post-Baccalaureate Doctor of Pharmacy Graduate in Canada $(1999)^{39}$

\section{Outcome \#1-Meets Patients' Drug-Related Needs}

Graduates, in partnership with patients and other health care providers, use their knowledge and skills to meet patients' drug-related needs, with the objective of achieving optimal patient outcomes and maintaining or improving the patients' quality of life.

\section{Outcome \#2 - Assess Legal, Ethical and Professional Obligations}

Graduates will be able to contribute to the development of legal, ethical and professional standards.

\section{Outcome \#3 - Provide Drug and Drug Use Information and Recommendations}

Graduates provide information and recommendations to individuals and groups concerning drugs and drug use to ensure optimum and cost effective patient care and to promote health.

\section{Outcome \#4 - Educate About Drugs, and Drug Use and Health Promotion}

Graduates educate individuals and groups to encourage appropriate drug use and to promote health.

Outcome \#5 - Apply the Principles of Scientific Inquiry to Contribute to the Profession and Society

Graduates will apply the principles of scientific inquiry to address pharmacy practice problems. 


\section{Appendix S1 (continued)}

\section{Educational Outcomes for First Professional Degree Programs in Pharmacy in Canada $(2014)^{40}$}

Care Provider: Pharmacy graduates use their knowledge, skills and professional judgement to provide pharmaceutical care and to facilitate management of patient's medication and overall health needs.

Communicator: Pharmacy graduates communicate with diverse audiences, using a variety of strategies that take into account the situation, intended outcomes of the communication and the target audience.

Collaborator: Pharmacy graduates work collaboratively with teams to provide effective, quality health care and to fulfill their professional obligations to the community and society at large.

Manager: Pharmacy graduates use management skills in their daily practice to optimize the care of patients, to ensure the safe and effective distribution of medications, and to make efficient use of health resources.

Advocate: Pharmacy graduates use their expertise and influence to advance the health and well-being of individual patients, communities, and populations, and to support pharmacist's professional roles.

Scholar: Pharmacy graduates have and can apply the core knowledge and skills required to be a medication therapy expert, and are able to master, generate, interpret and disseminate pharmaceutical and pharmacy practice knowledge.

Professional: Pharmacy graduates honour their roles as self-regulated professionals through both individual patient care and fulfillment of their professional obligations to the profession, the community and society at large.

The collection of preceptor experiences encompassed post-baccalaureate PharmD degree educational outcomes related to meeting patient needs, assessing legal, ethical and professional obligations, andproviding drug and drug use information and recommendations (Outcomes 1, 2 and 3), and entry-to-practice PharmD degree educational outcomes related tocare provider, communicator and collaborator, professional, and scholar (Table 3). 
Table 3. Mapping of major categories of student performance described in clinical preceptor interviews to intended Doctor of Pharmacy post-baccalaureate and Entry-to-Practice educational outcomes ${ }^{39,40}$

\begin{tabular}{|c|c|c|}
\hline \multirow{2}{*}{ Major Category } & \multicolumn{2}{|c|}{ CCAPP EDUCATIONAL OUTCOMES } \\
\hline & Post-Baccalaureate PharmD & Entry-to-Practice PharmD \\
\hline \multicolumn{3}{|l|}{ KNOWLEDGE } \\
\hline Knowledge base & - & Scholar 6.1 \\
\hline Knowledge translation & Outcome 1.2-1.7 & $\begin{array}{l}\text { Scholar } 6.1 \\
\text { Care Provider 1.3-1.8 }\end{array}$ \\
\hline \multicolumn{3}{|l|}{ TEAM INTERACTION } \\
\hline Integration & Outcomes 3.2 (III) & $\begin{array}{l}\text { Collaborator } 3.1 .2,3.2 .1,3.2 .4 \\
\text { Communicator } 2.1 .1-2.1 .3,2.1 .5, \\
2.1 .6,2.3 .3 \\
\text { Professional } 7.5 .3\end{array}$ \\
\hline Contribution & Outcome 3.2 & $\begin{array}{l}\text { Care Provider 1.3-1.8 } \\
\text { Scholar 6.2.5 } \\
\text { Collaborator 3.1.2, 3.2.3 } \\
\text { Professional 7.1.2, 7.4.5 } \\
\end{array}$ \\
\hline \multicolumn{3}{|l|}{ MOTIVATION } \\
\hline Independence & & Professional 7.3, 7.4.4 \\
\hline Disposition & - & $\begin{array}{l}\text { Professional 7.3.2, 7.3.6 } \\
\text { Collaborator 3.2.1 }\end{array}$ \\
\hline \multicolumn{3}{|l|}{ SKILLS } \\
\hline Critical thinking & Outcome 3.2 & $\begin{array}{l}\text { Care Provider } 1.1 .1 \\
\text { Communicator } 2.1 .1,2.1 .2,2.1 .5, \\
2.1 .6,2.3 .1 \\
\text { Advocate } 5.2 .1,5.2 .2\end{array}$ \\
\hline Information management & Outcome 3.2 & Scholar 6.2.1-6.2.6 \\
\hline Organization & Outcome 1.1 & Manager 4.1 .1 \\
\hline PATIENT CARE & & $\begin{array}{l}\text { Care Provider } 1.1 .1 \\
\text { Communicator } 2.1 .1,2.1 .2,2.1 .5 \\
2.1 .6,2.3 .1 \\
\text { Advocate } 5.2 .1,5.2 .2\end{array}$ \\
\hline \multicolumn{3}{|l|}{ COMMUNICATION } \\
\hline Presentation/Written & Outcomes 1.8 and 4.2 & $\begin{array}{l}\text { Communicator 2.1.4, 2.2.1, 2.2.2, } \\
2.4 .2\end{array}$ \\
\hline Health care members & Outcome 1.4 (ii, vii), 2.2 & $\begin{array}{l}\text { Communicator } 2.1 .6,2.3 .1 \\
\text { Collaborator } 3.1 .2,3.1 .5,3.1 .8,3.3 .7\end{array}$ \\
\hline \multicolumn{3}{|l|}{ PROFESSIONALISM } \\
\hline Conduct & - & - \\
\hline Insight & - & Professional 7.3.8 \\
\hline
\end{tabular}


Post-baccalaureate programme Outcomes 4 and 5, relating to public health promotion and applying principles of scientific inquiry to the study of pharmacy practice issues and other research activities, were poorly covered. Missing elements from the entryto-practice pharmacy programme outcomes were those relating to manager and advocate. Conversely, clinical preceptors described additional dimensions used to form their overall categorisation of student performance that are not included in the educational outcomes outlined for pharmacy degrees in Canada, such as 'observed commitment to the learning experience as evident by timeliness in completing assigned tasks' and 'seeking and responding to feedback':

\begin{abstract}
"A good student meets the requirement - has good knowledge and makes good interventions, but an excellent student does above what is required and provides more services. They arrive early and leave late. I had one student arriving 2 hours before the rotation [day] even started." [Preceptor C in Lebanon]

"For an average student, I am expecting that there is going to be some defense. You know, I had given feedback before [and the response was], '...well, this was happening or I was busy doing this...' etc... And I said, 'ok, I understand that does happen, but I am just telling you for your own knowledge, that you maybe prioritize your time effectively in a different manner'. Whereas an excellent student would take that, and I would see an immediate change."[Faculty Preceptor D in Qatar]
\end{abstract}

\title{
Discussion
}

Our study adds to the growing body of work exploring constructs formed by health professionals participating in student experiential training and provides additional elements that pertain to the comparability of assessment in cross-border curricula. Like North American practice-based supervisors in social work and medicine, these clinical pharmacy preceptors identified motivation and positive attitudes towards the learning experience as favourable student traits. Pharmacists in all regions conveyed iterations of 'above and beyond' when recalling the performance of exemplary students. Similarly, they expressed willingness to discount certain deficiencies if they believed students were making concerted efforts and subsequently made discernable progress. In many regards, these may be considered acceptable judgements, especially early in experiential training when students are given their initial opportunities to observe, participate and learn. ${ }^{42}$ Clinical preceptors may appreciate disappointing performance in isolation, rather than as a collection of consistent experiences with the student that were detrimental to the patient. Yet, contrasting evaluative thresholds for poor student performance represent a noted concern in the context of cross-border curricula. ${ }^{3}$ Advancing a student with real deficits in knowledge or skills that may jeopardise patient safety compromises professional responsibilities, but such deficits were not among the behaviours discounted by any pharmacist we interviewed.

Participant responses to interviewer prompts were spontaneous and, reassuringly, student features prioritised by these clinical preceptors engaged in pharmacist training based on the same curriculum outcomes overlapped. As we hypothesised, certain differences could be anticipated given the contexts from which they arose, but were overall fewer than anticipated. For example, Arab preceptors often highlighted student attire as contributing to their overall impression of the student. How a student dresses may be an indicator of his or her respect for authority in this region, in which a marked hierocracy of power and honour- 
based culture prevail. Thus, a well-presented student will reflect positively on the clinical preceptor. Conversely, Canadian preceptors placed greater emphasis on the critical appraisal skills demonstrated by their students. The concept of evidence-based medicine in patient care decision making has been in place substantially longer in North America than in the Middle East. These subtle differences may arise from dissimilarities in organisational structure or divergence in teaching environments across these settings. ${ }^{17}$ However, such differential salience is not unique to cross-cultural perspectives and may be widely found among single-context evaluators. ${ }^{4}$

Rater idiosyncrasy did not appear to be exacerbated by cultural context in our study; objectionable and exemplary student behaviours were similarly recognised and categorised in Canada and the Middle East. Recent studies make clear that clinical preceptors each possess a schema of expectations developed within their environment and rely on comparisons against these criteria to form and apply judgements. ${ }^{16,17}$ Although lamented, this recognised variability among supervisors within singular programmes in the same country stimulates ongoing scholarly inquiry, but does not generally undermine the integrity of the student's training experience. Conversely, transplanted curricula may be subject to reservations associated with the capability to uphold the indigenous programme's rigour and reputation. ${ }^{4}$ However, study of rater cognition has demonstrated how disparate frames of reference used by Western preceptors influence WBA. Trainees may be characterised according to the rater's perceptions of his or her own skills and knowledge, those of colleagues, and even preconditioned by the antecedent student. ${ }^{17,27,43}$ The exemplars of student performance formed in part from prior participation in clinical supervision serve as useful inventories to facilitate the differentiation of trainees. ${ }^{16,44}$ Our subjects in the Middle East had served as clinical faculty staff for an average of at least 5 years, but an initial cohort of experienced preceptors in other nascent partnerships may be lacking.

As globalised medical education findings to date rest largely on reviews of the experiences of members of the 'home' institution or the approaches employed by 'host' countries to deliver Western-oriented instructional strategies, understanding of how perceptions of student performance may vary among preceptors recruited in different countries is an essential part of quality assurance for programmes that use transplanted or shared curricula. Steps to anticipate and moderate socio-cultural aspects of student assessment during experiential training will contribute to successful internationalisation of health professional programming. Exploration of how clinical preceptors in Canada and the Middle East conceptualise different levels of student performance contributes to the development and interpretation of other prospective cross-cultural inquiry within non-experiential education settings, such as OSCEs and simulation-based assessments. ${ }^{45,46}$

Our findings support the contention that educational outcomes in the health professions require continual review and may benefit from an upheaval in paradigms. As in medicine and social work, descriptions of pharmacy student performance from both regions did not map well to all intended abilities outlined by the governing educational body. ${ }^{25,35}$ Clinical preceptors discussed student responses to feedback and self-reflection, whereas health professionals in other studies have pointed out ability to empathise. ${ }^{23,35,42,47}$ Greater integration of practice-based value judgements of the relevant competencies for 
assessments will be necessary to continue the development of contemporary health professional curricula. As in medicine, pharmacists in our study underscored how the student impacted on their day-to-day practice, describing the student's performance favourably if he or she worked independently and appropriately with entrusted tasks. Trust in a student to deliver patient care is an underlying feature prioritised across all disciplines and pharmacy education can be expected to follow medicine's lead towards the use of entrustable professional activities as assessment outcomes. ${ }^{35,42,47,48}$ Increased emphasis on narrative in evaluation might complement this transition. Detailed narratives or 'thick descriptions' may justify why preceptors assign their trust to students in unsupervised activity. ${ }^{49,50}$ However, reliance on comments to distil student performance remains inexact as written evaluations are subject to linguistic strategies used by preceptors to convey meaning in non-literal, socioculturally acceptable ways, a phenomenon not yet explored outside North America or Europe. ${ }^{22}$ Recommendations to revise conventional WBA scales and anchors to better align with the evaluative constructs that resonate with a profession's clinical faculty staff and the purposeful scrutiny of rater comments should therefore be undertaken within the overseas supervisory and patient care contexts of transplanted curricula., $26,51,52$

The limitations of our study may be considered to be similar to those inherent to other quantitative investigations. Our results are based on a small sample of clinical preceptors' experiences in supervising PharmD students. The documented perspectives are unique to these pharmacists and may not be widely generalisable, although the congruity of ideas across participants from different regions, as well as findings from research among other health professions, is a validating element. Nevertheless, these judgements are based on particular students whom these clinical preceptors had actually trained and the repository of these student experiences may not represent the multiplicity of performances or behaviours. By virtue of their programmes' shorter existence, Middle East participants had less to draw upon in this regard. We asked participants to frame perspectives according to experiences in training students in a post-baccalaureate programme. Given how clinical preceptors seem to partially construct criteria and make assessments against their other supervisory experiences, managing expectations of professionals training students in environments in which discipline-specific entry-to-practice outcomes and associated credentials are in transition from the baccalaureate level (doctor of pharmacy, masters of physiotherapy, as examples) must be a consideration. When the clinical preceptors interviewed offered features of student performance that fell below expectations, we did not prompt them further to determine if the pharmacist had failed these students. This is an important distinction to consider in the future because it pertains to whether students who are poorly perceived may benefit from redress if preceptors believe them to be unfit for practice. 


\section{Conclusions}

Our research findings are congruent with current literature related to the constructs used by clinical supervisors in health professional student WBA and provide additional insights comparing cross-national perspectives among preceptors participating in transplanted curricula. Like physicians and social workers, pharmacists form overall student impressions based on performance and behaviours that are not necessarily outlined in intended educational outcomes. Further study of how evaluation instruments and associated processes can integrate these judgements should also be pursued in this discipline. 


\section{References}

1. Harden RM. International medical education and future directions: a global perspective. Acad Med. 2006; 81: S22-29

2. Miller-Idriss C, Hanauer E. Transnational higher education: offshore campuses in the Middle East. Comp Educ. 2011; 47: 181- 207.

3. Waterval DGJ, Frambach JM, Driessen EW, Scherpbier AJJA. Copy but not paste: a literature review of crossborder curriculum partnerships.J Stud Int Educ. 2014; 19: 65- 85.

4. Waterval DG, Frambach JM, Oudkerk Pool A, Driessen EW, Scherpbier AJ. An exploration of crossborder medical curriculum partnerships: balancing curriculum equivalence and local adaptation. Med Teach. 2016; 38: 255-262.

5. Gosselin K, Norris JL, Ho MJ. Beyond homogenisation discourse: reconsidering the cultural consequences of globalised medical education. Med Teach. 2016; 38: 691-699.

6. Tsai SL, Ho MJ, Hirsh D, Kern DE. Defiance, compliance, or alliance? How we developed a medical professionalism curriculum that deliberately connects to cultural context. Med Teach. 2012; 34: 614-617.

7. Stevens FC, Simmonds Goulbourne JD. Globalisation and the modernisation of medical education. Med Teach. 2012; 34: e684-689.

8. Heffernan T, Morrison M, Basu P, Sweeney A. Cultural differences, learning styles and transnational education. J High Educ Policy Manag. 2010; 32: 27- 39.

9. McConnell MM, Harms S, Saperson K. Meaningful feedback in medical education: challenging the 'failure to fail' using narrative methodology. Acad Psychiatry. 2016; 40: 377-379.

10. Ginsburg S, Eva K, Regehr G. Do in-training evaluation reports deserve their bad reputations? A study of the reliability and predictive ability of ITER scores and narrative comments. Acad Med. 2013; 88: 1539-1544.

11. Epstein RM. Assessment in medical education. New Engl J Med. 2007; 356: 387- 396.

12. Dudek N, Marks M, Wood T, Lee A. Assessing the quality of supervisors' completed clinical evaluation reports. Med Educ. 2008 42:816-822.

13. Dudek NL, Marks MB, Bandiera G, White J, Wood TJ. Quality in-training evaluation reports--does feedback drive faculty performance? Acad Med. 2013;88:1129-1134.

14. Crossley J, Johnson G, Booth J, Wade W. Good questions, good answers: construct alignment improves the performance of workplace-based assessment scales. Med Educ. 2011; 45: 560- 569.

15. Holmboe ES, Huot S, Chung J, Norcini J, Hawkins RE. Construct validity of the mini clinical evaluation exercise (miniCEX). Acad Med. 2003; 78: 826-830. 
16. Govaerts MJ, van de Wiel MW, Schuwirth LW, van der Vleuten CP, Muiitjens AM. Workplace-based assessment: raters' performance theories and constructs. Adv Health Sci Educ Theory Pract. 2013; 18: 375-396.

17. Yeates P, O'Neill P, Mann K, Eva K. Seeing the same thing differently. Mechanisms that contribute to assessor differences in directly observed performance assessments. Adv Health Sci Educ Theory Pract. 2013; 18: 325- 341.

18. Pelgrim EAM, Kramer AWM, Mokkink HGA, van der Vleuten C. Quality of written narrative feedback and reflection in a modified mini-clinical evaluation exercise: an observational study. BMC Med Educ. 2012; 12: 97.

19. Littlefield JH, Darosa DA, Paukert J, Williams RG, Klamen DL, Schoolfield JD. Improving resident performance assessment data: numeric precision and narrative specificity. Acad Med. 2005; 80: 489- 495.

20. Cohen GS, Blumberg P, Ryan NC, Sullivan PL. Do final grades reflect written qualitative evaluations of student performance? Teach Learn Med. 1993; 5: 10-15.

21. Ginsburg S, Regehr G, Lingard L, Eva KW. Reading between the lines: faculty interpretations of narrative evaluation comments. Med Educ. 2015; 49: 296- 306.

22. Ginsburg S, van der Vleuten C, Eva KW, Lingard L. Hedging to save face: a linguistic analysis of written comments on in-training evaluation reports. Adv Health Sci Educ Theory Pract. 2015; 21: 175-188.

23. Ginsburg S, Mcllroy J, Oulanova O, Eva K, Regehr G. Toward authentic clinical evaluation: pitfalls in the pursuit of competency. Acad Med. 2010; 85: 780-786.

24. Rawlings A, Knox AD, Park YS, Reddy S, Williams SR, Issa N, Jameel A, Tekian A. Development and evaluation of standardised narrative cases depicting the general surgery professionalism milestones. Acad Med. 2015; 90: 1109-1115.

25. Rosenbluth G, O'Brien B, Asher EM, Cho CS. The 'zing factor' - how do faculty describe the best paediatrics residents? J Grad Med Educ. 2014; 6: 106-111.

26. Govaerts $M$, van der Vleuten CPM. Validity in work-based assessment: expanding our horizons. Med Educ. 2013; 47: 1164- 1174.

27. Gingerich A, Kogan J, Yeates P, Govaerts M, Holmboe E. Seeing the 'black box' differently: assessor cognition from three research perspectives. Med Educ. 2014; 48: 1055- 1068.

28. Anderson C, Bates I, Brock T, Nelson Brown A, Bruno A, Futter B, et al. Needs-based education in the context of globalisation. Am J Pharm Educ. 2012; 76: 1- 3.

29. Kane T. A clinical encounter of East meets West: a case study of the productions of 'American-style' doctors in a non-American setting. Global Stud J. 2009; 2: 12.

30. Al-Wassia R, Hamed O, Al-Wassia H, Alafari R, Jamjoom R. Cultural challenges to implementation of formative assessment in Saudi Arabia: an exploratory study. Med Teach. 2015; 37:S9-S19. 
31. Epstein J, Santo RM, Guillemin F. A review of guidelines for cross-cultural adaptation of questionnaires could not bring out a consensus. J Clin Epidemiol. 2015; 68: 435-441.

32. Austin Z, Ensom MH. Education of pharmacists in Canada. Am J Pharm Educ. 2008; 72: 128.

33. Wilbur K, Paiva M, Black EK. Pharmacy student and preceptor impressions of faculty liaison visits to experiential training sites. Am J Pharm Educ. 2015; 7: 134.

34. Smith A. Post-baccalaureate PharmD programmes for Canadian pharmacists: options and considerations. Can J Hosp Pharm. 2014; 67: 313-314.

35. Bogo M, Regehr C, Woodford M, Hughes J, Power R, Regehr G. Beyond competencies: field instructors' descriptions of student performance. J Soc Work Educ. 2006; 42: 579593.

36. Fusch PI, Ness LR. Are we there yet? Data saturation in qualitative research. Qual Rep. 2015; 20: 1408-1416.

37. Sbaraini A, Carter SM, Evans RW, Blinkhorn A. How to do a grounded theory study: a worked example of a study of dental practices. BMC Med Res Methodol. 2011; 11: 128.

38. Breckenridge J, Jones D, Elliott E, Nichol M. Choosing a methodological path: reflections on the constructivist turn. Grounded Theory Rev. 2012; 11: 64- 71.

39. Association of Faculties of Pharmacy of Canada. Educational Outcomes for a PostBaccalaureate Doctor of Pharmacy Graduate in Canada. Toronto, ON; 1999.

40. Canadian Council for Accreditation of Pharmacy Programs. Accreditation Standards for the First Professional Degree in Pharmacy Programs. Toronto, ON; 2014.

41. Royal College of Physicians and Surgeons of Canada. The CanMEDS 2005 Physician Competency Framework. Ottawa, ON; 2005.

42. DeBrew JK, Lewallen LP. To pass or to fail? Understanding the factors considered by faculty in the clinical evaluation of nursing students. Nurse Educ Today. 2014; 34: 631636.

43. Yeates $P, O^{\prime}$ Neill P, Mann K, Eva KW. 'You're certainly relatively competent': assessor bias due to recent experiences. Med Educ. 2013; 47: 910-922.

44. Berendonk C, Stalmeijer RE, Schuwirth LW. Expertise in performance assessment: assessors' perspectives. Adv Health Sci Educ Theory Pract. 2013; 18: 559-571.

45. Wilby K, Black E, Khalifa S, Makhalalati B, Austin Z, Aboulsoud S. Cumulative OSCE in Qatar: a pilot project for the Middle East. Abstract presented at the Australia and New Zealand Association for Health Professional Educators-Asian Medical Education Association (ANZAHPE-AMEA), 29 March to 1 April 2015, Newcastle, NSW.

46. Williams B, Abel C, Khasawneh E, Ross L, Levett-Jones T. Simulation experiences of paramedic students: a cross-cultural examination. Adv Med Educ Pract. 2016; 7: 181186. 
47. Regehr G, Ginsburg S, Herold J, Hatala R, Eva K, Oulanova O. Using 'standardised narratives' to explore new ways to represent faculty opinions of resident performance. Acad Med. 2012; 87: 419- 427.

48. ten Cate $O$, Chen HC, Hoff RG, Peters $H$, Bok $H$, van der Schaaf M. Curriculum development for the workplace using entrustable professional activities (EPAs): AMEE Guide No. 99. Med Teach. 2015; 37: 983-1002.

49. Hodges B. Assessment in the post-psychometric era: learning to love the subjective and collective. Med Teach. 2013; 35: 564- 568.

50. Durning SJ, Hanson J, Gilliland W, McManigle JM, Waechter D, Pangaro LN. Using qualitative data from a programme director's evaluation form as an outcome measurement for medical school. Mil Med. 2010; 175: 448-452.

51. Crossley J, Jolly B. Making sense of work-based assessment: ask the right questions, in the right way, about the right things, of the right people. Med Educ. 2012; 46: 28- 37.

52. Hanson JL, Rosenberg AA, Lane JL. Narrative descriptions should replace grades and numerical ratings for clinical performance in medical education in the United States. Front Psychol. 2013; 4: 668. 



\section{CHAPTER 3}

\section{Workplace-Based Assessment in Cross-Border Health Professional Education}

Published as: Wilbur K, Driessen EW, Scheele F, Teunissen PW. Workplace-Based Assessment in Cross-Border Health Professional Education. Teach Learn Med 2019;32:91-103.

DOI:10.1080/10401334.2019.1637742 


\begin{abstract}

\section{Construct}

The globalization of health care has been accentuated by the export of health professional curricula overseas. Yet, intact translation of pedagogies and practices devised in one cultural setting may not be possible or necessarily appropriate for alternate environments. Purposeful examination of workplace learning is necessary to understand how the source or "home" program may need adapting in the distributed or "host" setting.
\end{abstract}

\title{
Background
}

Strategies to optimize cross-border medical education partnerships have been largely focused on elements of campus-based learning. Determining how host clinical supervisors approach assessment in experiential settings within a different culture and uphold the standards of home programs is relevant given the influence of context on trainees' demonstrated competencies. In this mixed-methods study, we sought to explore assessor judgements of student workplace-based performance made by preceptors sharing a pharmacy curriculum in Canada and Qatar.

\section{Approach}

Using modified Delphi consensus technique, we asked clinical supervisors in Canada $(n=18)$ and in Qatar $(n=14)$ to categorize trainee performance as described in sixteen student vignettes. The proportion of ratings for three levels of expectation (exceeds; meets; or below) was calculated and within-country group consensus achieved if the level of agreement reached $80 \%$. Between-country group comparisons were measured using Chi square statistic. We then conducted follow-up semi-structured interviews to gain further perspectives and clarify assessor rationale. Transcripts were analyzed using thematic content analysis.

\section{Findings}

The threshold for between-country group differences in assessor impressions was met for only two of the sixteen student vignettes. Compared to Canadian clinical supervisors, relatively more preceptors in Qatar judged one described student as "meets" rather than "exceeds" expectations and one as "meets" rather than "falls below" expectations. Analysis of follow-up interviews exploring how culture may inform variations in assessor judgments identified themes associated with the: profession; organization; learner; and supervisor performance theories, but not their particular geographic context. Clinical supervisors in both countries were largely aligned in expectations of student knowledge, skills, and behaviours demonstrated in patient care and multidisciplinary team interactions.

\section{Conclusions}

Our study demonstrated variation in student assessment was more frequent among clinical supervisors within the same national context than any differences identified between the two countries. In these program settings, national socio-cultural norms did not predict global assessor impressions or competency-specific judgments; instead professional- and organizational-cultures were more likely to inform student characterizations of performance in workplace-based settings. Further study situated within the specific experiential learning contexts of cross-border health professional curricula is assuredly warranted. 


\section{Introduction}

Globalization of health is advancing as patients, providers, and even diseases increasingly cross borders. ${ }^{1}$ Medical education has accentuated this internationalization of care as health professional curricula are exported overseas. ${ }^{2,3}$ Knowledge and skill transfer is in part accomplished when universities establish branch or satellite campuses hosted in foreign settings or conversely, when domestic institutions adopt international programs or curricula. These particular cross-border education models have typically mobilized curricula from "Western" sources to other regions, but this orientation is diversifying. ${ }^{4} 5$ Benefits of these academic relationships include the provision of high-caliber content and training to placebound students who will join the workforce within 'host' countries as well as the extension of a 'home' institution's expertise and global identity.

Yet, intact translation of pedagogies devised in one cultural setting may not be possible or necessarily appropriate for alternate environments. ${ }^{6-9}$ Disparate learner attributes have been shown to influence adoption of necessary communication, group work, and selfdirected study behaviours. ${ }^{10-13}$ Local Instructors have generally had no input into the source curriculum and may face challenges acclimatizing to, for instance, student-centered didactic approaches. ${ }^{13,14}$ Recommendations to mitigate host faculty and student "culture shock" are increasingly informed by various international program experiences within campus-based learning spaces, but how clinical supervisors and trainees approach teaching and assessment in workplace-based settings is less well anticipated and described.

The mini-CEX assessment strategy has been embraced internationally, but has thus far been subjected to minimal prospective scrutiny for relevance prior to use in non-Western settings. ${ }^{15}$ The underlying premise of the mini-CEX is clinician provision of formative feedback based on observed trainee engagement in a patient encounter. ${ }^{16}$ However, cross-discipline studies demonstrate different cultural norms and preferences in feedback processes that may belie the fundamental aspects of this assessment method. Studies have found medical students and residents in Indonesia and Thailand place greatest value on the feedback from specialist physicians and clinical faculty emphasize feedback for corrective measures. ${ }^{17,18}$ Little has been reported on how such distinct culturally influenced preferences impact other aspects of workplace-based learning and assessment.

In the experiential training component of exported curricula, concerns may arise regarding divergence in local clinical supervisors' teaching and assessment of trainees and ability to conform to intended standards. ${ }^{4,19}$ These supporting faculty members have usually qualified in different academic settings and have not previously mentored students within the hosts' educational model. ${ }^{6,20,21}$ As such, these partners are asked to participate in processes with which they are less familiar or may have difficulty adopting. It may be foreseeable then that culture influences how clinical faculty supervise students and conduct workplace-based assessment (WBA). While disparate aspects of rater judgment are increasingly acknowledged, existing studies of how clinical supervisors form impressions and make, express, and interpret trainee performance is predominantly derived from Western settings. ${ }^{22-24}$ Gaining greater understanding of assessment decision-making of clinical supervisors among different cultural contexts is particularly relevant for programs delivering 
cross-border curricula whereby academic partners strive to maintain source standards and align with local outlooks.

In the present study, we used a comparative education approach to explore assessor judgements of student performance during the experiential training component of a shared cross-border curriculum. ${ }^{25}$ The clinical supervision of workplace-based learning in such international education models is usually locally situated in geographically and often culturally distinct regions. Members of a culture are considered to possess to some extent a collective identity, common history and experience, and shared beliefs, values, and norms. ${ }^{26}$ Socio-cultural theory in part assumes the perspective that context, including environmental, social, and cultural cues, shapes the behaviours of its constituents. ${ }^{27}$

However, culture is not only bound by geographic perimeters. Distinctions may also exist within a profession in that self-concept and roles are constructed and evolve under local conditions. ${ }^{28}$ In turn, an organization's culture is in part perpetuated through formal and informal practices of such individuals. ${ }^{29}$ Clinical supervisors within various contexts may be expected to perceive trainee performance through pluralistic cultural perspectives yielding dissimilar assessment. The existence or potential relevance of discrepancies between "host" and "home" preceptor standards for learners in the clinical component of cross-border curricula is largely unknown. Using a culturalist framework, we therefore sought to determine assessor impressions of student workplace-based performance in two different countries and explore culturally-informed variations in assessor judgments.

\section{Methods}

\section{Study Setting}

The Doctor of Pharmacy (PharmD) program is a post-baccalaureate degree supporting the training of graduate students to assume advanced pharmacy practice positions as integrated members of multidisciplinary teams delivering direct patient care. Two Canadian institutions offer this degree, as well as the College of Pharmacy (CPH) at Qatar University (QU) in Doha, Qatar. Established in 2007, QU CPH is not a satellite college of another affiliateuniversity or "branch campus" as previously described, but its professional degrees are accredited by the Canadian Council of Accreditation of Pharmacy Programs (CCAPP). ${ }^{30}$ Therefore, the mobilized curriculum is devised to meet the educational outcomes associated with Canadian pharmacist competencies for practice: Care Provider, Communicator, Collaborator, Leader/Manager, Scholar, Health Advocate, and Professional roles. ${ }^{31}$ Campusbased courses are delivered by an international faculty recruited to the university. Like students enrolled in the CCAPP-accredited programs in Canada, pharmacy students in Qatar complete a minimum of 40 weeks of experiential training over the course of their program to meet degree requirements. Since 2012, pharmacy students have joined local practice settings for required workplace-based learning under the supervision of predominantly Arab adjunct clinical faculty.

The cultural characterization of specific contexts has been heavily informed by Hofstede's theoretical framework for studying a nation's profile and making cross-cultural 
comparisons. ${ }^{32}$ According to this framework, effects of a society's culture on its members' values are outlined by six domains (power distance, individualism, uncertainty avoidance, masculinity, long term orientation, and indulgence) and may be used to study contrasts between countries according to their relative distinctions. Arab and North American societies such as Qatar and Canada, respectively, are typically positioned at opposite ends of described spectrums in power distance (for instance, high vs low acceptance of inequalities in authority), individualism (low vs high emphasis on independence and achievement of personal goals), and uncertainty avoidance (high vs low affinity for rigid infrastructure and rules). Originally developed through survey of how workplace values within the computer industry were influenced by culture, Hofstede's cultural dimensions concept has been widely applied in other fields, including health-related research pertaining to patient behaviours, provider care, and medical education. ${ }^{33-36}$ While these cultural dimensions are considered group-level constructs, the framework is useful to advance the understanding of how specific cultural orientations may shape workplace-based assessment.

\section{Study Design and Methodology}

To explore how clinical supervisors in Canada and Qatar characterize trainee performance, we used student descriptions in a format comparable to the clinical vignette, a short and purposefully written description of a person or a situation conceived to simulate a real world scenario. ${ }^{37-39}$ For our study, we developed student vignettes according to our prior findings of clinical supervisors in Canada and the Middle East who gave personal accounts of students (nameless) they had supervised during the experiential training component of North American-accredited pharmacy programs. ${ }^{40}$ We devised 16 student vignettes to describe pharmacist trainees who fell below, met, or exceeded expectations according to the 7 major categories of performance outlined in these interviews (Table 1). The descriptions were further framed for consideration as students who had completed two-thirds of the training program. The student vignettes were reviewed for content and clarity by two pharmacy preceptors in Canada and two in Qatar (Supplemental Material).

Using these student vignettes, we employed a mixed-methods design incorporating Delphi consensus measurement (quantitative) and interview techniques (qualitative) to explore assessor impressions across cultural contexts. The Delphi process was used to first elicit the supervisor characterizations of student performance, followed by interviews to gain greater insight and understanding of assessor rationale for these judgements. Data was collected between November 2016 and May 2017. The study was approved by the Qatar University Institutional Review Board (QU-IRB 613-E/16). 
Table 1. Major categories arising from interviews with PharmD preceptors discussing graduate students meeting, exceeding or falling below expectations

\begin{tabular}{ll} 
Major Category & Definition \\
\hline $\begin{array}{l}\text { KNOWLEDGE } \\
\text { Knowledge base }\end{array}$ & $\begin{array}{l}\text { Demonstrates ability to recall disease processes and } \\
\text { therapeutic knowledge (including but not limited to, } \\
\text { treatment alternatives, doses, mechanisms of action, } \\
\text { anticipated side effects) }\end{array}$ \\
Knowledge translation & $\begin{array}{l}\text { Applies information and knowledge to specific patient- cases } \\
\text { Retains learned information from earlier in the rotation for } \\
\text { future use in care }\end{array}$
\end{tabular}

\section{TEAM INTERACTION}

Integration

Swiftly adapts to the care setting and work environment; exhibits good judgment in timing recommendations and is accepted by the team

Contribution

Contributes to direct patient care, including evidence-based patient decision-making discussions at bedside rounds; decisions are based on good judgment; does not avoid responsibility for care

\section{MOTIVATION}

Independence

Disposition

SKILLS

Critical thinking

Information management

Organization
Proactively approaches work (patient care, assignments) with minimal prompting; demonstrates advance and on-going selfstudy/preparation; anticipates tasks and often provides extra efforts in assigned tasks

Exhibits enthusiasm, and curiosity in patient care and the learning process; preceptor and team members enjoy working with and teaching them

Demonstrates systematic process in patient-care and decision-making; can adapt accordingly when new information is provided; judgments are appropriate and safe

Efficiently retrieves and accurately appraises literature associated with drug therapy and patient management for their own learning and others' learning and decision-making purposes

Handles and prioritizes multiple tasks; punctual with assignments

Interacts comfortably with patients; caring attitude; seeks to inform and advocates improved patient management

\section{COMMUNICATION}

Presentation/Written
Creative and informative means to share information (presentation); clearly and succinctly summarizes information (presentation/chart note/written assignments) 
Health care members

Can comfortably and appropriately engage in polite disagreements related to patient-care and decision-making; shares information with preceptor and other health professionals in clear and succinct fashion

\section{PROFESSIONALISM}

Conduct

Insight
Respects preceptor expertise and time; demonstrates commitment to the rotation (arrives early and leaves late); appreciates learning opportunities

Reflects and responds to constructive feedback; never overconfident; recognizes limitations

\section{Quantitative Data Collection and Analysis}

We first explored clinical supervisor judgments using modified Delphi technique, a method used to systematically determine how well experts agree on a particular issue or idea. ${ }^{41}$ Features of this structured survey process include participant anonymity, iterative and controlled feedback by a central facilitator, and aggregation of responses. We invited pharmacists who have served as a clinical supervisor to at least 6 pharmacy trainees in domestic or international Canadian-accredited programs to participate in this study. These pharmacists from across Canada $(n=18)$ and Qatar $(n=14)$ who supervise post-baccalaureate Doctor of Pharmacy trainees assessed these student vignettes, presented randomly through a web-based platform, and were asked to categorize the descriptions according to their perceptions of the outlined performances or behaviors: 1) exceeds; 2) meets; or 3) falling below their expectations. The process was conducted separately for supervisors in Canada and Qatar. In the subsequent Delphi round of review, aggregate results (by country) were shared with its contributors who could then alter their own initial choices.

The proportion of ratings for the three expectation categories for each description was calculated and collated for each country. Within-country group consensus of student vignette categorization was reached if the level of agreement among the participants was equal to or greater than $80 \% .{ }^{42}$ Between-country group comparisons of student vignette characterization by clinical preceptors in Qatar and in Canada were made using Chi-square statistic. The significance level was set at $5 \%$ and therefore the null hypothesis of no difference between-country groups would be rejected at $p$-values less than 0.05 . Although we set quantitative thresholds for clinical supervisor agreement (within-country) and comparisons (between-country), we also used this method to scan for variability in collective judgments to guide selection of specific student vignettes suitable for qualitative analysis in the second part of our study.

\section{Qualitative Data Collection and Analysis}

We next invited these supervisors from the two countries to participate in follow-up interviews to gain further perspectives and clarify the rationale for their characterizations of the student vignettes. To obtain maximum contrasts of views, we included participants of 
both genders representing a range of supervisory experience in both specialized and general, teaching and non-teaching patient care settings. Ten clinical supervisor interviews ( $\mathrm{n}=5$ Qatar and $n=5$ Canada) were conducted by the first researcher (KW) between March and May 2017 and lasted approximately one hour.

Based on outcomes of the Delphi rounds completed 2-10 weeks earlier, participants were presented with a collection of the sixteen student narratives principally chosen according to between-country group differences and low levels of within-country group consensus. We asked participants to read each student vignette aloud and share any thoughts evoked by the given passage or section just spoken. If participants remained quiet after reading a section or conversely, completed several passages before pausing to verbalize their impressions, they were prompted by the researcher.

At the conclusion of each students' vignette reading, the researcher posed questions to gain further understanding of the participant's earlier statements. Discussions began iteratively, focusing on topics verbalized during participant reading of the student vignettes and then proceeded to specific questions structured to explore underlying cultural inferences or cues in these responses and informed in part by Hofstede's constructs of a society's cultural dimension. The interview guide was piloted with the same four pharmacists who reviewed and characterized the student vignettes, as described earlier. These individuals did not subsequently participate in the study.

All interview encounters were recorded and transcribed verbatim for analysis using thematic content analysis. ${ }^{43}$ Transcripts were read through several times and subjected to more detailed coding. Analysis began with open coding whereby the text was broken down by words or phrases into meaningful units. This initial coding was conducted with no sensitizing concepts apart from described content of the student narratives.

Using comparisons across transcripts, open codes were refined and combined into groups comprising potential major themes. The research team used a sample of three transcripts to generate an initial framework for thematic analysis. The coding scheme was further developed through additional open coding and constant comparison for fit or divergent cases. The first researcher (KW) then coded all data using the agreed upon framework. She is a pharmacist familiar with experiential training in both settings having worked in both Canada and Qatar for nearly 10 years each. The balance of the research team (an educationalist active in education across different cultures (ED) and two physicians and medication education researchers in workplace-based learning (FS, PT)) provided additional outside perspectives. Coding and analysis was supported by qualitative data analysis software (QSR NVivo for MAC version 10). 


\section{Results}

\section{Quantitative Results}

Findings from the modified Delphi process to determine the level of agreement of trainee characterization between clinical supervisors in Canada and Qatar are presented in Table 2. Consensus judgments were obtained for twelve (vignette numbers 1-4,7-9,11-14,16) and seven students (vignette numbers 2,4,7,8,11,14,16) among the Canadian and the Qatar preceptors, respectively. Although there was less within-country group agreement among the Middle East preceptors, the characterizations of several students matched those made by the Canadian clinical supervisors. For example, most clinical supervisors in Qatar also rated vignettes 1 and 12 as 'meets' and vignette number 9 as 'exceeds' expectations, but these proportions did not quite meet the $80 \%$ threshold for consensus. Both Canadian and Qatar preceptors were essentially split between 'meets' and 'exceeds' expectations for student vignettes number 5 and 6. One-third of Canadian and Qatar preceptors rated vignette number 10 as 'below' expectations. Most clinical supervisors characterized vignette number 15 as 'exceeds' expectations with fewer rating as 'meets'; one Qatar preceptor rated this student as 'below' expectations.

The threshold for between-country group differences in assessor impressions was met for two out of the 16 student descriptions (vignette numbers 3 and 13). When compared to preceptors in Canada, relatively more Qatar clinical supervisors judged the described student in vignette number 3 as meets rather than exceeds expectations and the student in vignette 13 as meets rather than falls below expectations (Table 2).

Based on these results, student vignettes representing both discrepant and consensus trainee characterizations within and across the clinical supervisory groups were chosen for further review in participant interviews. 
Table 2. Clinical Supervisor Characterization of Student Vignettes

\begin{tabular}{|c|c|c|c|c|c|c|}
\hline \multirow{2}{*}{$\begin{array}{l}\text { Student } \\
\text { Vignette }\end{array}$} & \multicolumn{2}{|c|}{$\begin{array}{l}\text { Below } \\
\text { Expectations }\end{array}$} & \multicolumn{2}{|c|}{$\begin{array}{l}\text { Meets } \\
\text { Expectations }\end{array}$} & \multicolumn{2}{|c|}{$\begin{array}{l}\text { Exceeds } \\
\text { Expectations }\end{array}$} \\
\hline & $\begin{array}{l}\text { Qatar } \\
(\%)\end{array}$ & $\begin{array}{l}\text { Canada } \\
(\%)\end{array}$ & $\begin{array}{l}\text { Qatar } \\
(\%)\end{array}$ & $\begin{array}{l}\text { Canada } \\
(\%)\end{array}$ & $\begin{array}{l}\text { Qatar } \\
(\%)\end{array}$ & $\begin{array}{l}\text { Canada } \\
(\%)\end{array}$ \\
\hline 1 & 14.3 & 5.9 & 78.6 & 82.4 & 7.1 & 11.7 \\
\hline 2 & 100 & 100 & 0 & 0 & 0 & 0 \\
\hline 3 & 13.3 & 0 & 40 & 5.9 & 46.7 & 94.1 \\
\hline 4 & 100 & 100 & 0 & 0 & 0 & 0 \\
\hline 5 & 0 & 0 & 40 & 47.1 & 60 & 52.9 \\
\hline 6 & 6.7 & 0 & 40 & 58.8 & 53.3 & 41.2 \\
\hline 7 & 93.3 & 100 & 0 & 0 & 6.7 & 0 \\
\hline 8 & 87.5 & 100 & 6.3 & 0 & 6.2 & 0 \\
\hline 9 & 0 & 0 & 37.5 & 11.7 & 62.5 & 88.3 \\
\hline 10 & 33.3 & 35.3 & 66.7 & 64.7 & 0 & 0 \\
\hline 11 & 13.3 & 17.6 & 86.7 & 82.4 & 0 & 0 \\
\hline 12 & 7.1 & 0 & 78.6 & 100 & 14.3 & 0 \\
\hline 13 & 66.7 & 100 & 33.3 & 0 & 0 & 0 \\
\hline 14 & 92.9 & 100 & 0 & 0 & 7.1 & 0 \\
\hline 15 & 7.1 & 0 & 21.4 & 23.5 & 71.5 & 76.5 \\
\hline 16 & 80 & 88.3 & 20 & 11.7 & 0 & 0 \\
\hline
\end{tabular}

Qualitative Results

Student descriptions in vignette numbers 5, 6, and 15 were broadly blueprinted favourably across the seven major categories of workplace-based performance and behaviours (Table 1). When explored in follow-up interviews, features pushing judgements to 'exceeds' expectations for student vignette number 5 included his or her continuous progression built upon an apparent strong knowledge base (for preceptors in Canada) or his or her technologic acumen (for preceptors in Qatar). Raters from both countries who retained 'meets' expectations for both vignette numbers 5 and 6 did so because of a lack of a "wow factor" (QAT 2) or not going "above and beyond" (CAN 5). Preceptors also wanted more information about the complexity of the recommendations and care the student was providing. The difference between meeting and exceeding preceptor expectations for the student outlined in vignette number 15 was varied. This student was characterized as "doing everything the way we hope it would be done" by one Canadian preceptor assigning the performance as "exceeds", whereby a Qatar preceptor rating as "meets" also conceded the performance was as expected, but not doing "something more". 
All preceptors interviewed seemed to struggle judging the student described in vignette number 10 , leading to discrepant judgements. The outlined unsatisfactory performance in knowledge application, independence, and critical thinking skills were sufficient for preceptors in Qatar and Canada to rate 'below' expectations. However, some clinical supervisors in both countries teased out the positive aspects of this trainee's motivation towards care and attitude with regard to feedback. Given the student's point in their experiential training curriculum (one-third remaining) coupled with a sense of responsibility to coach that the description seemed to evoke, some raters eventually settled upon a 'meets' expectation.

Like student vignette numbers 5, 6, and 15, vignette number 3 was also blueprinted favourably across the seven major categories of workplace-based performance and behaviours (Table 1). Nevertheless, unlike their Canadian counterparts, Qatar preceptors conservatively characterized as 'meets' (and on occasion) 'below' expectations. Some indicated doubt regarding the appropriateness of the independence demonstrated and wanted to know more about the student's application of primary literature to the care decisions described. Conversely, vignette number 13 outlined a student with practically global poor performance, yet some preceptors in Qatar allowed who the student seemed "hardworking" and "smart" but required confidence to unlock their true capabilities.

Data analysis of these interviews exploring how culture may inform variations in assessor judgments, and the sources for both within- and between-country groups, suggests that the societal norms typically associated with these two distinct geographic contexts plays little role. Instead, we identified themes of assessor influences associated with the: (1) profession; (2) organization; (3) learner; and (4) supervisors themselves.

\section{Professional Culture}

Clinical supervisors in Canada and Qatar expected pharmacist trainees to demonstrate the same fundamental professional skills and abilities. Examples frequently cited included interviewing patients to obtain medication histories or conducting medication counseling, interpreting laboratory and clinical data to assess safety and effectiveness of drug therapy, or critically appraising primary literature to deliver evidence-based care. Knowledge was valued, but must be coupled with application under specific, and often changing, patient conditions.

"So, they may say a better beta blocker is one of the three keys to heart failure management, but do they have the ability to provide nuance and is this for sure what's got to be done? In this patient this might be the exception..?" (CAN 5, student vignette 5)

"The student is too rigid and too fixed in his or her mind and whatever they've learned or whatever they feel is appropriate is stuck in their head." (QAT 2, student vignette 13)

Trainees were expected to be proficient communicators. The ability to convey care was emphasized in that students must listen with empathy and address individual and family concerns and needs with respect. 
"It's a very important part as your job as a pharmacist - to listen - if you are looking the other way, the patient can take it like a sign of disrespect." (QAT 4, student vignette 1)

"When they're trying to communicate with families, I would say they would minimally require preceptor guidance." (CAN 1, student vignette 1)

In Qatar, mixed-gender dynamics occasionally arise, but do not appear to aversely influence care or the learning experience. For example, male trainees are expected to interact with female patients, but must be mindful of their comfort or preference.

\section{Organizational Culture}

Clinical supervisors in both countries expected pharmacist trainees to readily establish their role in the care environment. Students interact directly with patients, but also collaborate with multidisciplinary colleagues to devise and execute care plans to achieve therapeutic goals. Mere physical presence during bedside rounds was insufficient; trainees characterized as meeting expectations contributed information when patient care discussions and decisions are taking place. Students should be capable to adapt their treatment plan when new data and perspectives emerge during these conversations. Supervisor concerns arose if treatment recommendations were not clearly communicated or supported by evidence.

"We are part of the team, so we are not there for only observation. We have to share our opinion... our experience, our knowledge." (QAT 3, student vignette 16)

"If they're not providing recommendations and they're just agreeing with what the physician says when it's not the correct or maybe the best therapy, to me that's below expectations for our rotation in my unit."(CAN 4, student vignette 16)

Such patient care input carries greatest weight if the trainee has formed positive working relationships with other care providers. All clinical supervisors highlighted the importance of "team fit" - what this means and how it arises. The recurring implication of cultivating a favourable team dynamic was the trainee's ability to mitigate and navigate disagreement with prescribers. Clinical supervisors wanted students to balance the confident pursuit of rational medication-related care decisions with collegial respect.

"She needs to be able to be the patient's advocate and I think that should be her driving force - of not only just the physicians are right." (QAT 5, student vignette 13)

"I don't see them advocating for the patient here, that they're reactive and what would concern me is ...if there were a critical medication-related problem I have no confidence that this pharmacist would act on it in order to achieve the patient's best outcome." (CAN 5, student vignette 13)

In this instance (vignette 13), lack of patient advocacy contributed to unanimous below expectation rating by Canadian preceptors unlike a third in Qatar who felt the student could still meet expectations. 
Each clinical preceptor could describe hierarchal structures in their teams - "yeah, there is a pecking order" (CAN 1). While non-confrontation and maintenance of group harmony are ideals typical to Qatar, clinical supervisors identified why trainees should not be dissuaded from expressing dissenting opinions.

"Consultants and clinical pharmacists know that they can work together and when they disagree, that's fine and that's why we are here. We don't disagree to just fight; we are trying to improve patient care." (QAT 4, student vignette 9)

\section{Learner Attributes}

Clinical supervisors in both countries considered generic student traits when rating trainee performance and behavior. Supervisors appreciated students who appeared open to receiving constructive feedback and used it to improve their performance. Other information useful for student characterization included demonstrated organizational skills. These were deemed essential to safe patient care, forecasted presumed trainee abilities once they reached the workforce, and in Qatar, was often associated with others' perception of the profession as a whole (not of the student alone).

The trainee's ability to manage multiple tasks and patient cases frequently factored into preceptor judgments. Clinical supervisors in Canada and Qatar rated 'below expectations' students who they could not trust to appropriately deliver unsupervised care.

"... if they were not identifying all the relevant clinical data and modifying their daily plans, so sort of not really on top of things... the preceptor couldn't be as confident and might have to start ...taking a second look at the patients." (CAN 3, student vignette 9)

"He has daily progress notes, he reviews it regularly, he works independently...so he took charge of the patient care and he understands that he is accountable for the patient." (QAT 4, student vignette 3)

However, the appropriateness of independent work was important. Across the different performance descriptions, preceptors in both countries outlined potential caveats ("do they overrate their abilities?" CAN 2 or "[she's] ultimately under the responsibility of the preceptor" QAT 3) and uncertainty about such work outlined in vignette 3 contributed to lower characterization of this student among a minority of Qatar raters.

In Qatar and Canada, clinical supervisors commended students who appeared committed to patient care and the learning experience, but were also wary of the nature of this "extra work".

"Showing that they are willing to go beyond what is actually required of them... it shows that they are dedicated and doing it for their own interest as well." (QAT 2, student vignette 12)

"I don't know... when they stay too late in the evening, does that mean that they're not using their time effectively?" (CAN 3, student vignette 13) 


\section{Supervisory Performance Theories}

Pharmacists in Qatar and Canada revealed variable performance theories about trainee supervision, specifically for those students with perceived described deficiencies. Preceptors in both countries set clear thresholds for an overall student characterization of "below expectations" when certain competencies were not yet readily observed at the identified point in their training program. Meanwhile, other clinical supervisors offered "allowances" for initial poor performance when coupled with agreeable trainee behaviours (e.g. "working hard", possessing a positive attitude, demonstrating progress) and judged them more leniently. Supervisors outlined coaching tactics they might implement for these trainees who were not meeting their expectations.

"...transitioning from a student to a pharmacist you will have this confidence issue especially challenging people who have been in the field for many years and maybe specialists and whatever else ... maybe the preceptor could assist her with speaking up during the rounds and asking on every case 'Do you have a comment?'... I think she just needs a bit of a push on that." (QAT 2, student vignette 12)

"Confidence seems to be the issue and they might need some role modeling; they might need a fair amount of support here. So I think this is a real opportunity to work with this individual."

(CAN 5, student vignette 13)

\section{Discussion}

This study exploring cross-cultural differences in the workplace-based assessment of pharmacist trainees shows that clinical supervisors expect similar standards of performance and behaviours irrespective of the geographic context. We found high level of agreement between preceptor characterization of students described in the vignettes and largely concordant views on what shaped these impressions in the follow-up interviews. Discrepant assessor judgments anticipated by Qatar's and Canada's contrasting predominant societal norms and values were not borne out in our analysis. Middle East supervisors encouraged independence in patient care, discounted some elements of team harmony, and expected trainees to challenge physician authority when appropriate, which may be considered at odds with regional positions in dimensions of uncertainty avoidance, individualism, and power distance, respectively. The cultures influencing assessor judgments were instead more likely to be associated with the professional practices and organizational environments in which these students and preceptors are situated.

Student rating agreement between clinical supervisors in our study could in part be attributed to an unforeseen shared professional culture. Middle East pharmacy education programs are typically drug product-oriented and reinforce the view of the pharmacist as a scientist. ${ }^{44}$ In North America however, pharmacists continue to assume expanded scopes of patient-centered practice, including independent prescribing authority. ${ }^{45} \mathrm{How}$ is it then that these pharmacists' characterization of trainees in culturally-distinct contexts is congruent?

Lave and Wenger have previously described distributed communities of practice - groups not constrained by time or place. ${ }^{46}$ Pharmacists recruited as clinical supervisors in Qatar are 
those who often engage with the profession internationally (e.g. conference attendance, society membership) and may have also had additional advanced practice training from "Western" institutions themselves. The PharmD program at Qatar University has additionally invested in various preceptor development strategies and exposes practicebased faculty to curricular expectations through invited participation in campus-based student assessments. ${ }^{20,47,48}$ Unlike incomparable faculty knowledge and skills detected in other cross-border curricula, these clinical supervisors understand and accept the intended professional competencies taught in a Canadian-oriented program and therefore their judgments of trainee performance may be uniquely aligned with North American counterparts. ${ }^{6}$

Curriculum equivalence of cross-border health professional education can be thwarted by gulfs between home and host health care systems and therefore the backdrop for workplace-based learning. ${ }^{6,13}$ If authentic experiences and interactions are necessary for optimal clinical learning, then training sites must facilitate inclusion. ${ }^{49,50}$ Like Canadian preceptors, Qatar clinical supervisors unfavorably characterized students who did not actively participate in therapeutic decision-making. Despite the lingering entrenched roles of physicians as the uncontested leads in Middle East patient care settings, these preceptors expect trainees to collaborate with multidisciplinary colleagues and respectfully debate inappropriate prescribing. However, such expectations are not necessarily typical for pharmacist trainees in the region. ${ }^{51}$ It would seem then that a local organizational culture exists where the legitimacy of pharmacists and trainees alike is recognized and disruptions to usual hierarchies is permitted.

How these conditions have developed in Qatar is unclear. However, the country is host to North American branch campuses training almost a dozen other health professionals (e.g. medicine, nursing, respiratory therapy), some of which have been in place for more than 15 years. $^{52}$ It is possible that the confluence of students from these programs converging at these clinical teaching sites, and associated practice-faculty development, has in some way altered the culture of institutional health care training and customarily scripted disciplinary roles. Furthermore, the pharmacists in our study appear to have cultivated microenvironments of advanced practice which they closely curate; participants from Qatar more often emphasized concern for how trainee performance and behaviour would reflect upon the profession as a whole.

Ideally, clinical supervisors facilitate student learning by enabling patient care activities while at the same time recognizing and accommodating individual trainee needs and abilities. However, the supervisory efforts expended seem to have implications on the student impressions they form. Ginsburg et al have previously described how medical residents who consume staff attention could be perceived as "problematic". ${ }^{53}$ In Bogo's work with social work field supervisors, excessively needy students strained relationships. ${ }^{54}$ We did not set out to expressly study the clinical teaching attitudes or behaviours of our sample population, but evidence of "supervisory cultures" surfaced during participant interviews. In both Canada and Qatar, a number of preceptors expressed commitment to coach certain students who struggle to meet their expectations. However, the extent of such supervisory allowances was not uniform, as others were resolute in expectations of self-directed learning and 
independent work. Opportunity for trainees to function as independent clinicians is fundamental to the experiential element of health professional curricula. Qatar clinical supervisory preferences for student autonomy in a cultural setting where unstructured situations elicit discomfort and decision-making is often centralized are noteworthy.

Through our mixed-methods approach, we were able to corroborate results of assessor impressions in Canada and in Qatar. Quantitatively, we illustrated within-country group disagreement in student ratings among clinical supervisors before making comparisons between countries. Qualitatively, we used the student vignette approach employed by others exploring trainee characterization by clinical supervisors in social work and medicine. ${ }^{37,55}$ In this way, we further support our findings of cross-border consistency; preceptor judgments are also similar in how they are dissimilar. The basis of within-group differences were often shared by pharmacists in Canada and in Qatar and according to assessment phenomenon previously described. We too found how preceptors in both countries often agreed with one another about described or observed student performance and then made contrasting judgements according to their own benchmarks and priorities for a trainee's work and patient care. ${ }^{22-24}$ We are mindful that lack of the within-group consensus in our Delphi results obscured abilities to ascertain statistically significant differences in our between-country comparison. Nevertheless, in our in-depth qualitative analysis, we were still unable to uncover specific cultural influences on rater expectations as framed by Hofstede's theoretical theory.

The vignettes created for our study were informed by accounts of student performance and behaviours previously offered by clinical supervisors in Qatar and Canada. Although implicit messages are often embedded in such narratives' language, assessors are still able to consistently interpret descriptions and characterize students despite unfamiliarity with local contexts. ${ }^{56}$ However, this method could fall short as a suitable surrogate for impressions formed following actual direct observation of trainees. We intentionally used gender-neutral written descriptions to eliminate potential visual cues that could contribute to unconscious rater bias and reassuringly, student vignettes did resonate with the participants. Clinical supervisors are nevertheless more accustomed to making judgments of trainees with whom they spend time. Although preceptors were comfortable arriving at the requested summative assessment, participants did on occasion in the interview component indicate a desire for information not described in the student vignette in order to arrive at a more fully informed assessment characterization.

Albeit the predominant cultural theory, Hofstede's framework is not without detractors. ${ }^{57}$ McSweeney has put forth one of the most compelling criticisms challenging the suitability of using individuals' survey responses to represent an entire nation's values and the reductionism of complex behaviours into five finite dimensions. ${ }^{58}$ Jones additionally invokes the dynamic influence of the prevailing political climate at the time of data collection (1960's). ${ }^{59}$ Irrespective of methods or era, most subsequent models of national culture have in fact arrived at comparable descriptive constructs to understand and contrast cultures and Hofstede himself reemphasizes how the validity of his labeled dimensions have withstood repeated measurements over time across broad fields of study. ${ }^{60,61}$ We are comfortable with the suitability of Hofstede's cultural dimensions as a means to explore assessor judgments 
in North American and Middle East contexts. The absence of discernable difference in student assessment by clinical supervisors in the two national settings that may have been anticipated according to this theory does not in it of itself further censure the framework. Indeed, we may have considered using Hofstede's cultural dimensions to determine cultural orientations at the individual level as other researchers have done. ${ }^{33}$

As this study of workplace-based assessment was conducted in a cross-border pharmacy education program in Qatar, our findings may not be transferable. The pharmacist training program under comparison with Canada is not a typical cross-border partnership with a branded home campus satellite. We are unable to explicitly mark how ours and the other actual branch health professional programs positioned in this host country have affected the local health system or training over time. "Learner-friendly" clinical environments may have evolved and therefore our findings are not necessarily reflective of clinical supervisor expectations in other Arab settings. Indeed, Hodges et al has called for a broader examination of the impacts of cross-border medical education programming on local healthcare systems and care providers, but little work has yet been done in this regard. ${ }^{62}$ Researchers might consider the transferability of our results to the practice environment in other settings, within the Middle East or otherwise, taking into account their own health profession's cross-border curriculum and workplace-based context.

\section{Practical Implications}

Continued growth of global partnerships in health professional education necessitates ongoing quality assurance. While prior work has described cross-border curriculum implementation experiences, ours is the first study to examine workplace-based assessment of enrolled students training in domestic practice environments. In this particular geographic setting, the tensions inherent between local adaptation and preservation of program standards as it pertains to workplace-based assessment would appear to be reconciled. Specifically, national societal norms were not associated with global assessor impressions or competency-specific judgments; instead professional- and organizationalcultures were more likely to inform student characterizations. Further study of the experiential curriculum in this and other cross-border medical education partnerships is assuredly warranted and should incorporate questions about other practical aspects of the workplace-based training, including student perspectives and how home programs influence host practice settings and the resultant institutional culture. 


\section{References}

1. Woodward D, Drager N, Beaglehole R, Lipson D. Globalization and health: a framework for analysis and action. Bulletin of the World Health Organization 2001;79:875-881.

2. Kosmutzky A, Putty R. Transcending borders and traversing boundaries: a systematic review of the literature on transnational, offshore, cross-border, and borderless higher education. J Stud Int Educ. 2016;20:8-33.

3. Altbach PG, Knight J. The internationalization of higher education: motivations and realities. J Stud Int Educ. 2007;11:290-305.

4. Zhang L, Kinser K, Shi Y. World economies and the distribution of international branch campuses. Int J High Educ. 2014;8:8-9.

5. Kahn HE, Agnew M. Global learning through difference: Considerations for teaching, learning, and the internationalization of higher education. J Stud Int Educ. 2017;21:5264.

6. Waterval DG, Frambach JM, Oudkerk Pool A, Driessen EW, Scherpbier AJ. An exploration of crossborder medical curriculum partnerships: Balancing curriculum equivalence and local adaptation. Med Teach. 2015;38:255-262.

7. Mackay $M$, Joyce-McCoach J, Stephens $M$, et al. Review of transnational nursing education programme curricula: process, findings, and recommendations. Nurse Educ Today. 2016;42:73-77.

8. Gosselin K, Norris JL, Ho MJ. Beyond homogenization discourse: Reconsidering the cultural consequences of globalized medical education. Med Teach. 2016;38:691-699.

9. Shams F, Huisman J. The role of institutional dual embeddedness in the strategic local adaptation of international branch campuses: evidence from Malaysia and Singapore. Stud High Educ. 2016;41:955-970.

10. Gwee MC-E. Globalization of problem-based learning (PBL): cross-cultural implications. Kaohsiung J Med Sci. 2008;24:S14-S22.

11. Frambach JM, Driessen EW, Chan LC, van der Vleuten CPM. Rethinking the globalisation of problem-based learning: how culture challenges self-directed learning. Med Educ. 2012;46:738-747.

12. Jippes M, Majoor GD. Influence of national culture on the adoption of integrated and problem-based curricula in Europe. Med Educ. 2008;42:279-285.

13. Waterval DGJ, Frambach JM, Driessen EW, Scherpbier AJJA. Copy but not paste: a literature review of crossborder curriculum partnerships. J Stud Int Educ. 2014;19:6585.

14. Waterval DG, Frambach JM, Driessen EW, Muijtjens A, Scherpbier AJ. Connected, attracted, and concerned: AQ study on medical crossborder curriculum partnerships. Med Teach. 2018;40:1293-9. 
15. Suhoyo Y, Schönrock-Adema J, Rahayu GR, Kuks JBM, Cohen-Schotanus J. Meeting international standards: a cultural approach in implementing the mini-CEX effectively in Indonesian clerkships. Med Teach. 2014;36:894-902.

16. Norcini JJ. The Mini Clinical Evaluation Exercise (mini-CEX). Clin Teach. 2005;2:25-30.

17. Suhoyo Y, van Hell EA, Prihatiningsih TS, Kuks JBM, Cohen-Schotanus J. Exploring cultural differences in feedback processes and perceived instructiveness during clerkships: Replicating a Dutch study in Indonesia. Med Teach. 2014;36:223-229.

18. Wong AK. Culture in medical education: comparing a Thai and a Canadian residency programme. Med Educ. 2011;45:1209-1219.

19. Segouin C, Hodges B, Byrne PN. World conference on medical education: A window on the globalizing world of medical education? Med Teach. 2007;29:e63-e66.

20. Wilbur K, Paiva M, Black EK. Pharmacy Student and preceptor impressions of faculty liaison visits to experiential training sites. Am J Pharm Educ. 2015;79:134.

21. Henderson D, McGrath PD, Patton MA. Experience of clinical supervisors of international medical graduates in an Australian district hospital. Aust Health Rev. 2017; 41:365-371.

22. Govaerts MJ, Van de Wiel MW, Schuwirth LW, Van der Vleuten CP, Muijtjens AM. Workplace-based assessment: raters' performance theories and constructs. Adv Health Sci Educ Theory Pract. 2013;18:375-396.

23. Gingerich A, Kogan J, Yeates P, Govaerts M, Holmboe E. Seeing the 'black box' differently: assessor cognition from three research perspectives. Med Educ. 2014;48:1055-1068.

24. Gauthier G, St-Onge C, Tavares W. Rater cognition: review and integration of research findings. Med Educ. 2016;50:511-522.

25. Phillips D, Schweisfurth M. Comparative and International Education: An Introduction to Theory, Method and Practice. New York, NY: Continuum International Publishing; 2006.

26. Sun S. Organizational culture and its themes. Int J Bus Manag. 2008;3:137-141.

27. Kozulin A. Vygotsky's educational theory in cultural context. Cambridge University Press; 2003.

28. Trede F, Macklin R, Bridges D. Professional identity development: a review of the higher education literature. Studies High Educ. 2012;37:365-384.

29. Schein EH. Organizational culture. Am Psychol. 1990;45:109-119.

30. The Canadian Council for Accreditation of Pharmacy Programs. Accreditation standards for the first professional degree in pharmacy programs. Toronto, ON; .2014.

31. Association of Faculties of Pharmacy in Canada (AFPC). Educational Outcomes for First Professional Degree Programs in Pharmacy in Canada. Ottawa, ON; 2017. 
32. Hofstede G. Culture's Consequences: Comparing Values, Behaviors, Institutions, and Organizations Across Nations. Second ed. Thousand Oaks CA: Sage Publications; 2001.

33. Wilby KJ, Govaerts MJ, Austin Z, Dolmans DH. Exploring the influence of cultural orientations on assessment of communication behaviours during patient-practitioner interactions. BMC Med Educ. 2017;17:1-10.

34. Verma A, Griffin A, Dacre J and Elder A. Exploring cultural and linguistic influences on clinical communication skills: a qualitative study of international medical graduates. BMC Med Educ. 2016;16:1-10.

35. Meeuwesen L, van den Brink-Muinen A, Hofstede G. Can dimensions of national culture predict cross-national differences in medical communication? Patient Educ Counsel. 2009;75:58-66

36. Deschepper R, Grigoryan L, Lundborg CS, et al. Are cultural dimensions relevant for explaining cross-national differences in antibiotic use in Europe? BMC Health Serv Res. 2008;8:123.

37. Regehr G, Ginsburg S, Herold J, Hatala R, Eva K, Oulanova O. Using "standardized narratives" to explore new ways to represent faculty opinions of resident performance. Acad Med. 2012;87:419-427.

38. Rawlings A, Knox AD, Park YS, et al. Development and evaluation of standardized narrative cases depicting the general surgery professionalism milestones. Acad Med. 2015;90:1109-1115.

39. Alexander CS, Becker HJ. The use of vignettes in survey research. Public Opin Q. 1978;42:93-104.

40. Wilbur K, Hassaballa N, Mahmood OS, Black EK. Describing student performance: a comparison among clinical preceptors across cultural contexts. Med Educ. 2017;51:411-422.

41. Clayton MJ. Delphi: a technique to harness expert opinion for critical decision-making tasks in education. Educ Psychol. 1997;17:373-386.

42. Powell C. The Delphi technique: myths and realities. J Adv Nurs. 2003;41:376-382.

43. Braun V, Clarke V. Using thematic analysis in psychology. Qual Res Psychol. 2006;3:77101.

44. Kheir N, Zaidan M, Younes H, El Hajj M, Wilbur K, Jewesson PJ. Pharmacy education and practice in 13 Middle Eastern countries. Am J Pharm Educ. 2008;72:133.

45. Donald M, King-Shier K, Tsuyuki RT, et al. Patient, family physician and community pharmacist perspectives on expanded pharmacy scope of practice: a qualitative study. CMAJ Open. 2017;5:E205.

46. Lave J, Wenger E. Situated learning: Legitimate peripheral participation. Cambridge University Press; 1991. 
47. Wilby KJ, Black EK, Austin Z, Mukhalalati B, Aboulsoud S, Khalifa SI. Objective structured clinical examination for pharmacy students in Qatar: cultural and contextual barriers to assessment. East Mediterr Health J. 2016;22:251-257.

48. Paravattil B, El Sakrmy N, Shaar S. Assessing the evidence based medicine educational needs of community pharmacy preceptors within an experiential program in Qatar. Curr Pharm Teach Learn. 2017 10:47-53.

49. Yardley S, Teunissen PW, Dornan T. Experiential learning: AMEE guide No. 63. Med Teach. 2012;34:e102-e115.

50. Strand $P$, Edgren $G$, Borna $P$, Lindgren S, Wichmann-Hansen G, Stalmeijer RE. Conceptions of how a learning or teaching curriculum, workplace culture and agency of individuals shape medical student learning and supervisory practices in the clinical workplace. Adv Health Sci Educ Theory Pract. 2015;20:531-557.

51. Kheir N, Al Saad D, Al Naimi S. Pharmaceutical care in the Arabic-speaking Middle East: literature review and country informant feedback. Avicenna. 2013:2.

52. Kane T. Higher education in Qatar: Does a US medical school break the baroque arsenal. In G Donn G and Y Al Manthri Y (Eds.), Education in the broader Middle East: Borrowing a baroque arsenal. Oxford Symposium Books; 2013.

53. Ginsburg S, Mcllroy J, Oulanova O, Eva K, Regehr G. Toward authentic clinical evaluation: pitfalls in the pursuit of competency. Acad Med. 2010;85:780-786.

54. Bogo M, Regehr C, Woodford M, Hughes J, Power R, B RG. Beyond competencies: Field instructors' descriptions of student performance. J Social Work Educ. 2006;42:579-594.

55. Bogo M, Regehr C, Power R, Hughes J, Woodford M, Regehr G. Toward new approaches for evaluating student field performance: tapping the implicit criteria used by experienced field instructors. J Social Work Educ. 2004;40:417-426.

53. Ginsburg S, van der Vleuten CPM, Eva KW. The hidden value of narrative comments for assessment: a quantitative reliability analysis of qualitative data. Acad Med. 2017;92:1617-1621.

54. Signorini P, Wiesemes R, Murphy R. Developing alternative frameworks for exploring intercultural learning: a critique of Hofstede's cultural difference model. Teach Higher Educ. 2009;14:253-6.

55. McSweeney B. Hofstede's model of national cultural differences and their consequences: A triumph of faith-a failure of analysis. Hum Relat.2002;55:89-118.

56. Jones M. Hofstede - Culturally questionable? Oxford Business \& Economics Conference. Oxford, UK, 24-26 June, 2007.

57. Magnusson P, Wilson RT, Zdravkovic S, Xin Zhou J, Westjohn SA. Breaking through the cultural clutter: a comparative assessment of multiple cultural and institutional frameworks. Int Mark Rev. 2008;25:183-201.

58. Hofstede G. Dimensions do not exist: A reply to Brendan McSweeney. Hum Relat. 2002;55:1355-61. 
Chapter 3

59. Hodges BD, Maniate JM, Martimianakis MA, Alsuwaidan M, Segouin C. Cracks and crevices: Globalization discourse and medical education. Med Teach. 2009;31:910-7. 


\section{Supplemental Materials Sixteen Student Vignettes Describing Pharmacy Student Workplace-based Performance}

\section{Student 1}

Throughout the rotation, B demonstrates full responsibility for the patients under B's care. B completes all the required tasks including daily review of the patient's progress through patient interview and review of the medical record. B follows-up on the status of all actions required by the pharmacist that was determined the previous day with the preceptor. Although $B$ misses some points when evaluating patient cases, $\mathrm{B}$ is able to identify the major and some minor drug-related problems. When interacting with patients and families, B demonstrates respect and communicates at an appropriate level; however, B doesn't always maintain eye contact while trying to take notes of the information and sometimes requires preceptor guidance.

B has a good basic knowledge in terms of pathophysiology, pharmacology and other disease related topics. However, B requires preceptor's help to guide how to apply this information in real practice. Once B is instructed, B is able to perform the required tasks appropriately. B is punctual in completing assignments, presentations, and other tasks requested by the preceptor; however, $B$ is not doing any work that is beyond what is required.

B gets along with other members of the department and the health care team. $B$ is respectful when trying to incorporate ideas into the team discussions, but most of the time the preceptor must encourage and prompt $B$ to engage in the decision-making process for patient care. When the preceptor provides $B$ with this prompting and makes other recommendations $B$ understands these weaknesses and tries to incorporate the feedback in subsequent daily work.

\section{Student 2}

$\mathrm{C}$ comes to the rotation with minimum preparation in terms of review of the posted learning objectives or basic information related to the care setting and associated patient conditions and therapy. C's ability to progress throughout the early part of the rotation is therefore lacking.

$\mathrm{C}$ does not seem to recognize C's limitations and believes to know more than $\mathrm{C}$ actually does. When the preceptor identifies shortcomings and means to improve, $C$ doesn't show any meaningful change or appear to take the preceptor feedback seriously. In terms of completing tasks, $\mathrm{C}$ is not organized and exhibits poor time management. $\mathrm{C}$ does not submit assignments on time and most often tries to find excuses, such as "the questions were too challenging"; " the time given was not enough"; or " । had other things to do/finish"

On a personal level, $\mathrm{C}$ is very affable and is able to build friendly relationships with those $\mathrm{C}$ encounters. $C$ seems to especially enjoy meeting and counseling patients and they recognize $C$ as a distinct member of their care team. However, C's approach to patient care is incomplete; C does not understand the main disease or drug-related issues of the patients under C's care and always misses important points. The preceptor is compelled to follow C's patients closely to ensure adequacy of care and this generates additional work for them.

Overall, $C$ does not actually seem to enjoy the work and the preceptor feels that $C$ would rather be elsewhere. The preceptor has the impression C's main goal is to just get past this rotation regardless of the learning benefit. 


\section{Student 3}

On the first day, D articulates well-written personal learning objectives and reviews their progress regularly with the preceptor throughout the rotation. Once oriented to the practice setting, D works independently to work-up the patients assigned. $D$ is punctual when it comes to assignments and arrives early to the practice area to ensure adequate preparation so as to be able to review the cases with the preceptor and to contribute to patient care during bedside rounds by making recommendations to the team.

D demonstrates broad knowledge of pathophysiology and therapeutic alternatives of conditions encountered in this specific patient care setting. D needs little preceptor support to fit this information with practical decision-making for patient's medications.

D gets along with everyone and is able to fit easily within the team, answering questions accurately and generally communicating very effectively. Nurses and medical students feel comfortable approaching D with questions: D will go to great lengths to find the answer even if it is not related to the care of D's own patients. D 's information is always trusted by the preceptor and the consultant physicians.

D is considered outstanding in several aspects and will always be remembered by the preceptor as well as other health care providers.

\section{Student 4}

From the beginning of the rotation, $F$ shows no interest in being there. $F$ does not engage in the process at all and misses many of the clinical rounds.

F seems to have numerous gaps in fundamental therapeutic knowledge and patient care processes and so the preceptor must spend more time with $\mathrm{F}$ compared to other students explaining the value of each step and directing $\mathrm{F}$ to do more self-directed reading to "catch up".

F generally misses the main drug-related problems and therefore cannot reliably assume the care of more than 2 or 3 patients throughout the whole rotation. F picks up on very simple problems to present them in front of the team and has made suggestions for care that may be considered unsafe and without prior review with the preceptor. As such, $\mathrm{F}$ is not included in the decision-making processes of most team members and all patient care questions are directed to the preceptor instead.

$\mathrm{F}$ is always late for meetings and cannot be depended upon to complete patient care tasks. The preceptor doubts that $F$ really wishes to be a pharmacist. The preceptor is trying to minimize any negative impact $F$ may have on their practice during the time $F$ is with them in their care setting. 


\section{Student 5}

$\mathrm{G}$ is a "technological whiz" - very comfortable with electronics. Storing all resources and notes on a tablet, $G$ can quickly access information to answer questions that arise during discussions with the preceptor. $\mathrm{G}$ is also generous in sharing useful tips new to the preceptor. This level of organization is also reflected in how $\mathrm{G}$ manages patient information. While $\mathrm{G}$ maintains thorough patient files, $\mathrm{G}$ can often accurately offer current and historical patient data without referring to notes.

Not surprisingly, $G$ is able to deliver dynamic presentations: the audio-visual content maintains the audience's attention, but $\mathrm{G}$ is also an accomplished presenter and is able to clearly and accurately answer questions from the audience.

$\mathrm{G}$ is enthusiastic in joining bedside rounds and will stay on with the medical team when the consultant conducts additional teaching for the residents and students once all patients have been reviewed. The preceptor does feel they must prompt $G$ to speak up during rounds to make and justify a recommendation and $G$ has instead been giving the ideas to the residents before or after in private discussions. However, by the last week of the rotation, $\mathrm{G}$ is showing more confidence and can make suggestions directly to the consultant.

Disease management discussions with $\mathrm{G}$ are always in-depth. Even when reviewing topics that have not yet been encountered in patients during the rotation, $\mathrm{G}$ takes the lead in describing the condition's etiology, pathophysiology, pharmacology of therapeutic alternatives, and key primary literature supporting care recommendations. The preceptor provides minimal prompting and the two engage in thoughtful dialogue regarding how the evidence is incorporated into actual practice.

G seems excited to be a pharmacist and projected a positive image for the pharmacy profession in all patient and care provider interactions. By the end of the rotation, the preceptor will miss G's contribution to the care setting and feels to have also learned a lot from this student. 


\section{Student 6}

From the very beginning of the rotation, it was clear to the preceptor that $\mathrm{H}$ was serious about the learning experience and asked many questions related to the processes of patient care. $\mathrm{H}$ was trying hard to match and apply the knowledge acquired from the campus-based curriculum to the care of patients on this preceptor's clinical service. $\mathrm{H}$ fit in well with the medical team and was always ready with suggestions for patient's medication. $\mathrm{H}$ was able to time the contributions to the team discussions to preserve the "flow" of bedside rounds and would wait until afterwards to ask the preceptor additional questions and seek input regarding ways "rejected" recommendations might be re-phrased in the future.

Near the end of the first week, the preceptor found out that $\mathrm{H}$ was gathering all patient information from secondary sources and had been reluctant to meet the patients personally. $\mathrm{H}$ had previously been on a critical care rotation where communication with intubated patients was limited. Once re-oriented to the importance of acquiring information directly from the patient to help inform medication decision-making and optimize care, $\mathrm{H}$ was making daily visits to patients and providing discharge counseling.

By the end of the rotation, $\mathrm{H}$ is assuming the care of most of the patients on the service. When reviewing the patients with the preceptor, $\mathrm{H}$ is prepared with all the pertinent information for each and has an outlined plan of action. $\mathrm{H}$ never mixes up the cases and generally knows who will be ready for discharge and has anticipated their medication counseling needs. The preceptor feels very confident in $\mathrm{H}^{\prime}$ s management and has largely left the service in $\mathrm{H}^{\prime}$ s hands by the end of the rotation. Having said this, $\mathrm{H}$ does not hesitate to consult with the preceptor when issues arise that $\mathrm{H}$ feels unable to handle alone.

In addition to the patient care responsibilities, the preceptor has given $\mathrm{H}$ some additional projects, including work on a formulary drug therapy review for the Drug \& Therapeutics Committee. The report was well written and $\mathrm{H}$ had the opportunity to present the findings in person at the committee meeting. With minimal preceptor coaching, $\mathrm{H}$ was able to clearly communicate the review contents and answer most of the questions put forward.

$\mathrm{H}$ was a likeable student and the preceptor enjoyed teaching experience with $\mathrm{H}$ on their rotation. 


\section{Student 7}

$\mathrm{J}$ is a very bright student who seems to easily retain new information and readily draw upon prior knowledge. $J$ is able to confidently discuss many aspects of the epidemiology and pathophysiology of diseases and the evidence behind treatment alternatives when participating in discussions with the preceptor. Unfortunately, this does not translate into the provision of patient care once they move to the ward. $J$ is disorganized with patient records and sometimes offers inaccurate patient information to the preceptor or team to cover for the lack of preparation before rounds. The preceptor is compelled to closely cover and restrict the number of patients assigned to $\mathrm{J}$.

Dedication to patient care and rotation tasks is also questionable. On more than one occasion, the preceptor has been unable to locate $J$ in the hospital during the usual working hours. When eventually reached, J comes up with several excuses ("I was feeling sick" or "my brother needed a drive home"). Agreed action plans for patient care (such as conducting discharge counseling or recommending modification of drug dosing) remain incomplete the next day when the preceptor checks. When such observations and feedback for improvement is offered, $\mathrm{J}$ is not receptive and tells the preceptor all prior clinical supervisors thought I was great.

Unlike other students, J does not prepare any handout materials for journal clubs and case presentation slides are poorly constructed. While J can cleverly respond to some of the audience follow-up questions, the preceptor feels embarrassed to be associated with J and regrets the time wasted trying to mentor $\mathrm{J}$.

\section{Student 8}

$\mathrm{K}$ makes an immediate initial favourable impression. Arriving early on the first day impeccably dressed, $\mathrm{K}$ has brought in homemade cookies for the pharmacy department. While it seems $\mathrm{K}$ did not have time to review the rotation goals and objectives, $K$ eagerly inquires about the preceptor's expectations and their planned workflow over the next weeks.

In the direct patient care area, K quickly gets to know the nurses and allied health staff. Before the first day is over, $\mathrm{K}$ has committed all the names to memory. By the end of the first week, $\mathrm{K}$ has only reviewed 2 cases (the service is 25 beds). Whenever the preceptor reaches the ward, $K$ can be found socializing with the nurses and medical students and is unprepared to discuss the patients and will try to shift the focus to something irrelevant (for example, asking what the preceptor has been working on in administrative and research roles). $\mathrm{K}$ is appropriate during team rounds but often cannot answer questions directly, offering easily accessible patient information unrelated to the question and already known to other members. $\mathrm{K}$ is not making a unique contribution to the patient's care. It seems $\mathrm{K}$ cannot (or chooses not to) effectively search for literature in electronic resources or databases, relying heavily on outdated clinical practice guidelines as references. K will promise to look something up or complete a task, but there is always some delay for even the most straightforward things.

During discussions, K can only offer superficial therapeutic information and lacks detailed knowledge usually demonstrated by members of $K^{\prime} s$ class who this preceptor has previously supervised. $K$ is incredibly pleasant to have around in the pharmacy department and respectful of the preceptor but there is no appreciable improvement in identified deficiencies after several episodes of verbal and written feedback. 


\section{Student 9}

Throughout the rotation, $\mathrm{L}$ has been an asset to the preceptor in the patient care area. $\mathrm{L}$ is on the ward very early in the morning to review new admissions and the progress of the existing patients. When the time comes for rounds, $L$ has the complete picture for the medication profile and the patient's response to therapy. $L$ has been warmly welcomed by the other health professionals on the service, not only for the thorough knowledge of the patients, but also due in part to L's easy-going, yet professional demeanor. $L$ contributes to the group discussions in a meaningful way, but also offers information to the medical residents beforehand $-L$ is not one to "show up" another member. As such, $L$ is often sought out by others for drug information and patient medication consultation, as well as the delivery of additional presentations to the team. $L$ gladly takes on these tasks. For a few days, $L$ took responsibility of a more junior pharmacy student who joined team rounds. This mentorship was a great support to the busy preceptor and $L$ seemed to genuinely enjoy it.

L's ability to identify relevant clinical and laboratory data and use it in modifying a daily plan for each patient gives the preceptor confidence to permit $L$ to care for the patients with minimal supervision. $L$ is conscientious of this responsibility and offers detailed patient updates at pre-determined times each day. $L$ will look to the preceptor for guidance in more complex cases, but will always have specific questions and considered potential solutions for discussion beforehand. The preceptor feels $L$ has used the experience to gain new knowledge and skills and would not hesitate to recommend $L$ to superiors if a position became available at the site after $L$ graduates.

\section{Student 10}

$M$ is a student who initially appears to have good potential. Throughout the rotation, $M$ is enthusiastic about learning the "ins and outs" of care in the practice setting and is always seeking preceptor feedback for improvement. $M$ is quickly accepted by the pharmacy staff and allied health members and they see how kind and conscientious $M$ is with the patients. $M$ spends significant time listening to patient concerns and preparing educational resources (like discharge medication lists or calendars) and takes the initiative to locate other health information on the patient's behalf (like how to make an appointment to see a dietitian or where they could join a smoking cessation clinic). When making recommendations, $\mathrm{M}$ is an advocate for expressed patient needs, but sometimes the urgency of these medication problems are not perceived by the prescriber as important as more life-threatening issues. $M$ does not always effectively justify the recommendations to be understood by the prescriber.

$M$ has some difficulty making progress early in the rotation. Despite spending time with the preceptor discussing specific topics with relevant case examples, $M$ continues to overlook these issues among the drug-related problems identified for the patients. The preceptor must correct $M$ several times before $M$ "catches on". $M$ is very diligent in accomplishing assignments, but has often set off in the wrong direction. $M$ will make a detailed, well-written, well-resourced report on something that was the opposite of what the preceptor actually asked. With redirection, $\mathrm{M}$ will eventually finish the task, but has wasted time focusing elsewhere. $M$ understands the preceptor's perspective in all feedback interactions and makes corrective effort. The preceptor spends time working on these retention and prioritization issues and M eventually "gets up to speed" by the end of the rotation. 


\section{Student 11}

Within the first week, $\mathrm{N}$ had settled in very well at the ambulatory care clinic. $\mathrm{N}$ learned the 'flow' of patient care quickly and adapted appropriately to the preferences of the different nurses and physicians. In the past, $\mathrm{N}$ had completed an inpatient clinical experience related to the diseases managed in the clinic and possessed good background information related to the disease and treatment. However, $\mathrm{N}$ was often unable to alter management recommendations to fit the specific needs to these patients during initial care plan discussions. Over several days, the preceptor had to prompt $\mathrm{N}$ to go back to review the primary literature, but by the end of the rotation, $\mathrm{N}$ demonstrated the ability to form relevant and evidence-based recommendations.

$\mathrm{N}$ communicates effectively with patients and is proficient at obtaining health and medication histories, conducting relevant physical exam, and providing clear medication counseling. $\mathrm{N}$ interacts appropriately with other clinic staff and shares pertinent patient information acquired during interactions. Following review with the preceptor, $\mathrm{N}$ is not reluctant to offer recommendations to prescribers, but only by the last week of the rotation is $N$ trusted by the preceptors the rotation to do so independently. Written documentation of recommendations in the patient's medical record requires ongoing practice in future rotations. Despite seeing models and reviewing preceptor feedback on drafts, $\mathrm{N}$ is not quite able to succinctly describe the care provided or clearly outline suggestions for patient monitoring.

$\mathrm{N}$ generally arrives each day organized for patient appointments and prepared for discussions with the preceptor and members of the clinic health care team. During quiet periods in the clinic, $\mathrm{N}$ does not seem to use the downtime well. Instead of using the opportunity to make progress on outstanding tasks, $\mathrm{N}$ will always wait for specific direction from the preceptor. $\mathrm{N}$ has been a nice and like-able student throughout and by the end of the rotation is mostly functioning as a capable contributor to the clinic. 


\section{Student 12}

$\mathrm{P}$ is a quiet and reserved student and demonstrates extensive knowledge related to treatments and diseases; however, $\mathrm{P}$ is very "by the book" and cannot handle ambiguity well. If there is not a definitive course of therapy supported by the evidence, $\mathrm{P}$ is incapable of arriving at a recommendation without the support of the preceptor. The preceptor attributes this partly to P's unfamiliarity to the practice area. The preceptor does observe how $P$ eventually becomes more comfortable in these circumstances and learns to draw upon the patient characteristics and other existing knowledge to make decisions. By the end of the rotation, $\mathrm{P}$ has an improved ability to prioritize drug-related problems and make relevant goals of therapy and monitoring plans, but this may be a challenge again when $P$ shifts to a different care setting.

P gets along with others and is always willing to "pitch" in when additional tasks arise, like when a team member is looking for drug information or last minute patient discharge counseling. There is never an occasion where $\mathrm{P}$ is unprepared for pre-arranged meetings with the preceptor to review patients or disease-related topics. P takes responsibility for the patients assigned and always treats them with care and respect. Every day, $\mathrm{P}$ is able to offer an update on their condition and has stopped by to speak briefly with them. $\mathrm{P}$ seems to function best in one-to-one situations and initially prefers making recommendations to prescribers following rounds. Unfortunately by then of course, treatment decisions have been made at the bedside. The preceptor spends additional time with $\mathrm{P}$ to review what $\mathrm{P}$ has planned and strategizing on when $\mathrm{P}$ may appropriately contribute the information during rounds.

$\mathrm{P}$ is open to feedback and is genuinely interested in how to improve. While it was not an outstanding rotation, the preceptor feels P's progress will continue as P gains greater confidence with more clinical experience. 


\section{Student 13}

$Q$ is a hard-working student. There is no spare moment that $Q$ is not found looking up and reading materials. $Q$ is usually reviewing patient profiles early in the morning and stays into the evening completing care assignments. $Q$ never resists taking on tasks given by the preceptor and the schedule of activities (like journal club or case presentations) proceeds as originally set out at the start of the rotation - there is no negotiation on the part of the student to shift deadlines.

Q attends rounds well-prepared, but will never oppose the team's therapeutic decisions. Instead Q consistently challenges the preceptor. Q politely resists the preceptor's identification of suboptimal or (sometimes) inappropriate patient management and is not open to exploring new ideas. $Q$ will repeatedly state that, "this is how I have seen it done elsewhere" or "the attending (physician) must know best". $Q$ is generally able to access primary literature with some prompting by the preceptor, but seems paralyzed to try to implement it in the care of the patients. Q seems to lack confidence in opposing the status quo and so the preceptor continues to round with the team throughout the rotation to model pharmacist contributions to care and coach $Q$ in this regard.

$Q$ is enthusiastic to take on the care of new cases and wishes to participate in the care of all the patients on the service (sometimes as many as 30), but does not always gather the pertinent data. Q seems more comfortable with discharge counseling than with initial patient interviews. The resulting initial patient work up then is consistently superficial and so the preceptor must insist that $Q$ take on fewer cases in order to reinforce a systematic process. $Q$ is initially frustrated with this but by the midpoint has recognized the value of this approach in the learning experience. By the end of the rotation, $Q$ is able to use a structured approach to gather information directly from some patients or family members. However, this seems uncomfortable for $Q$ and therefore $Q$ does not consistently develop a rapport with assigned patients throughout their hospital stay.

$\mathrm{Q}$ is a bright and capable pharmacist, but the preceptor is exhausted by the end of the rotation and is not sure what unique contribution to patient care $Q$ will make in the eventual workplace. 


\section{Student 14}

The rotation with $\mathrm{R}$ is difficult from the outset. On the first day, $\mathrm{R}$ showed up one hour late with no notice or apology for the preceptor. While never as blatantly late again, $R$ consistently missed the start of rounds by 10-15 minutes and left for home at the end of the day at the earliest possible opportunity.

$\mathrm{R}$ is not trustworthy in patient care. A treatment plan will be discussed between $\mathrm{R}$ and the preceptor prior to interacting with the team at rounds, but the preceptor will find out from the prescriber that $R$ recommended an opposite course of action. When confronted, $R$ will offer some excuse and imply that the preceptor themselves did not communicate the agreed plan clearly with $R$. $R$ is unable to effectively contribute to decision-making at the bedside as $\mathrm{R}$ is texting on the phone throughout rounds. When pointed out by the preceptor, $\mathrm{R}$ insists that it was to look up drug information or the practice guideline. Despite such affinity for the phone, the preceptor often has to page $\mathrm{R}$ several times before receiving a response.

$R$ does not always present to the workplace professionally attired and is immune to corrective feedback. When specific deficiencies are identified on the midpoint evaluation, $R$ is quick to complain that the rotation is too demanding given the additional course work $\mathrm{R}$ has to complete for the program outside of these clinical internships. The preceptor expresses genuine concern that $\mathrm{R}$ does not fully appreciate the importance of pharmacist responsibilities, but $R$ resists this feedback, reassuring the preceptor that since they were smart enough to get licensed, the situation cannot be as bad as the preceptor is making it out to be.

\section{Student 15}

S has an excellent pharmacotherapy knowledge base and knows how to apply it efficiently. When S question comes up during patient or therapeutic discussions with the preceptor or the medical team, $S$ takes the initiative to answer it and knows exactly how and where to find the information in a short time. S can apply S's existing and new knowledge to the care of the patient with minimal preceptor correction.

From the start of the rotation until the end, $\mathrm{S}$ demonstrates keen interest in the experience by taking every opportunity to learn more about the patient cases, both through asking questions about how the preceptor delivers care and by self-directed reading in the primary literature. S looks up additional topics that are not expressly related to the preceptor's or team's questions. S observes other health care professionals and works with them to improve the standard of care. The preceptor feels $S$ routinely goes above and beyond the outlined rotation expectations.

During rounds, $\mathrm{S}$ is able to identify all the drug related problems and prioritize them accordingly. $\mathrm{S}$ makes recommendations to the team that are patient-specific. S regularly interacts with patients and explains all the relevant information in a very clear, simple, and empathetic a manner.

$\mathrm{S}$ seeks feedback from the preceptor and other health care team. $\mathrm{S}$ always react positively to any criticism by trying to apply the recommendations for changes and improvement. $S$ is perceived by the preceptor as closest to being a pharmacist functioning independently in this care setting. 


\section{Student 16}

$\mathrm{T}$ is able to function in this care setting and complete primary tasks; however, $\mathrm{T}$ is less independent than some other students. T relies on the preceptor for guidance and instructions throughout the rotation with no appreciable change from the first few days.

$T$ can discuss disease states and medications with thorough and accurate information, including some primary literature. T can remember information discussed with the preceptor and use it to solve patient drug-related problems, when prompted. However, $T$ does not seem to have any interest in accomplishing tasks that are in addition to what is asked by the preceptor and does the minimum required to finish the rotation.

T seems to be more of an observer than a participant. T rarely takes initiative to do something and consistently waits for others to take the lead. While Thas a good "bedside manner" with patients and can devise rationale and safe medication plans when discussing the case with the preceptor, when it comes to interacting with other team members, $T$ is always reluctant to provide recommendations. $T$ agrees on whatever the physician said and does not question any decision.

$\mathrm{T}$ is generally a nice person and is always on time. When the preceptor provides feedback, T passively accepts the constructive criticism without argument or self-reflection. In general, $T$ has good knowledge but is lacking in drive. 



\section{CHAPTER 4}

\section{Team Member Expectations of Trainee Communicator and Collaborator Competencies}

Published as: Wilbur K, Teunissen PW, Scheele F,. Driessen EW. Team member expectations of trainee communicator and collaborator competencies - so shines a good deed in a weary world? Med Teach 2021 (early release online)

DOI:10.1080/0142159X.2021.1874325 


\section{Abstract}

\section{Background}

Workplace-based assessment may be further optimized by drawing upon the perspectives of multiple assessors, including those outside the trainee's discipline. Interprofessional competencies like communication and collaboration are often considered suitable for team input.

\section{Aim}

We sought to characterize multidisciplinary expectations of communicator and collaborator competency roles

\section{Methods}

We adopted a constructivist grounded theory approach to explore perspectives of multidisciplinary team members on a clinical teaching unit. In semi-structured interviews, participants described expectations for competent collaboration and communication of trainees outside their own discipline. Data were analyzed to identify recurring themes, underlying concepts and their interactions using constant comparison.

\section{Results}

Three main underlying perspectives influenced interprofessional characterization of competent communication and collaboration: 1) general expectations of best practice; 2) specific expectations of supportive practice; and 3) perceived commitment to teaching practice. However, participants seemingly judged trainees outside their discipline according to how competencies were exercised to advance their own professional patient care decision-making, with minimal attention to the trainee's specific skillset demonstrated.

\section{Conclusion}

While team members expressed commitment to supporting interprofessional competency development of trainees outside their discipline, service-oriented judgement of performance loomed large. The potential impact on the credibility of multidisciplinary sources for workplace-based assessment requires consideration. 


\section{Background}

Multiple perspectives of student performance observed over time can optimize assessment in competency-based health professional education. ${ }^{1,2}$ Frequent feedback accompanied by regular intervals of guided review and student self-reflection fosters learner progress and improvement within and across clerkship experiences. ${ }^{3}$ Trainees thereby also have longitudinal opportunity to demonstrate performance in diverse learning and patient care contexts. ${ }^{4}$ In these workplace-based settings, assessment is typically the responsibility of discipline-specific clinical supervisors, but students often train within various configurations of interprofessional teams in direct patient care settings. ${ }^{5}$ Learners exchange information with other professionals to determine and monitor patient status, formulate care plans, and execute treatment decisions, sometimes in larger formal group settings (e.g. rounds). Such proximity to trainee work uniquely positions team members from other disciplines to offer input on demonstrated competencies. Formative assessment by multidiscipline sources is particularly salient given these practitioners interact with trainees engaged in care that may have been assigned to be completed independently and not directly observed by the principal clinical supervisor. ${ }^{6,7}$

While different viewpoints may be enlisted to enrich assessment in competency-based education, doing so without a full appreciation of whether they arise from dissimilar complementary or contradictory-frames of reference may be problematic. Prior studies reveal discrepant interpretations of competency roles contributing to uncertainty and even conflict when health professionals embark upon the assessment of physicians-in-training. ${ }^{8-}$ 11 Sonnenberg et al unearthed distinct ways mixed-discipline pediatric team members viewed expectations of resident "leadership". ${ }^{10,11}$ Nurses, occupational therapists, social workers, speech language pathologists, and psychologists largely eschewed the notion of one formal (physician) leader coordinating patient care in favor of shared responsibilities. Burford et al identified how paramedic, podiatry, and occupational therapist educator views of what constitutes "professional" diverge. ${ }^{8}$ Their described discourses affirmed the complexities of stated competency constructs. Conflicting ideas and expectations about trainee performance also seem to be borne out in actual formal assessment practices. Psychometric analysis of submitted multisource feedback reports demonstrated how different disciplinary groups could not reliably assess "health advocate" roles of residents. ${ }^{9}$ Disparate interprofessional perspectives should be unsurprising given disciplinary boundaries are in part marked by "underlying theory and idealized models and exemplars" of performance for every discipline. ${ }^{12}$ Yet, our limited knowledge of how clinicians across professional contexts conceptualize and judge competencies for trainees outside their discipline may impair the opportunity for learners to profit from their insights.

Unlike discipline-specific knowledge and technical skills, communicator and collaborator roles have been considered transferable between professions and therefore germane for appraisal from multidiscipline sources. ${ }^{13}$ These competencies not only consistently appear in the educational outcomes for many health professions, but are predominantly featured in interprofessional education competency frameworks. ${ }^{14}$ For example, learners are expected to communicate in responsive and responsible ways and engage team members in order to support shared decision-making in practice. ${ }^{15}$ The interprofessional cooperation and 
respective application of discrete expertise deemed necessary to deliver quality patient care can also be utilized to enhance trainee learning experiences. ${ }^{16,17} \mathrm{It}$ is increasingly recognized that groups may inform more comprehensive student appraisals as a collective enhances the holistic representation of observed performances and behavior. ${ }^{18-22}$ However, the connotations of labelled competency roles may be considered socially constructed and culture-bound by profession; feedback of "shared" competencies is influenced by divergent education, training, and language as well as a clinician's social identity and how "outgroup" members are perceived. ${ }^{23-25}$ Paradoxically, it may be through dissimilar and potentially conflicting perspectives - and not simply the lens of a singular discipline - that student learning and assessment is augmented. ${ }^{26,27}$ These factors underscore the need to gain greater understanding of interprofessional viewpoints and how team members form impressions and respond when called upon to judge undergraduate trainees outside their discipline or specialty. In this study, we sought to shed light on multidisciplinary constructs of communicator and collaborator roles and associated feedback. Specifically, we addressed the questions: how do interprofessional team members expect trainees to demonstrate competent communication and collaboration; and what are their experiences offering feedback about trainees outside their discipline?

\section{Methods}

The research questions were explored using constructivist grounded theory, an approach which seeks to explain processes through the experiences of individuals within a particular social context. ${ }^{28}$ We conducted semi-structured interviews, a method particularly well-suited to gain access to individual perspectives and experiences of participants who represent different segments or types (e.g. health professions). Setting

This study took place at the second largest hospital in Canada. It is a 1,000-bed tertiary care centre offering specialized health services to individuals across the province of British Columbia. It is also the major teaching hospital affiliated with the University of British Columbia where students from nearly 100 different health professions and medical subspecialties train in multidisciplinary team contexts.

Acute medicine teams are comprised of a wide array of professionals (dietitians, nurses, occupational therapists, pharmacists, physicians, physiotherapists, social workers, speech language pathologists, spiritual care consultants) providing care to adult patients with complex general medical illnesses. Training on this service is also a core clerkship experience for students within these disciplines, as well as those of other consulting allied health care providers. Clerkship experiences may last a few weeks to several months, depending on the professional program. Acute medicine team members are exposed to many and varied health professional trainees in their day-to-day practice and often supervise workplacebased learning of students within their own discipline.

\section{Data Collection}

Members of the multidisciplinary team on the acute medicine units were invited to participate in this study. Consenting participants were drawn from a convenience sample of 
all care providers who had worked at least 12 months as part of the acute medicine team, purposefully sampling from the distinct disciplines to ensure a broad range of experiences. The interview guide was informed by the decision to explore the communicator and collaborator role expectations health professionals express for trainees from other disciplines joining the team. Two pilot interviews with volunteers representing the different disciplines in practice on the acute medicine units were conducted to test the length and wording of the interview guide; this data was not included in the study analysis. The interview began asking participants about expectations for trainees inside their own discipline and transitioned for stated expectations and experiences for trainees outside their profession, using pharmacy as an anchoring example. As a healthcare provider outside medicine and nursing, pharmacy trainees represent the largest group of allied health discipline learners populating the acute medicine workplace-learning environment. Questions next addressed in what mixed-discipline feedback opportunities participants engaged. We ultimately interviewed fourteen team members (3 dietitians, 3 nurses, 2 occupational therapists, 4 physicians, 1 physiotherapist, 1 social worker). Two of these physicians and one nurse were recruited to interview following preliminary data analysis to further inform refining of theoretical categories. Each discussion was audio-recorded and transcribed verbatim. The transcription was subsequently verified and finalized by a research assistant (RA) and one investigator.

\section{Data Analysis}

Interview data analysis was conducted iteratively in parallel with on-going data collection and employed the constant comparative approach of grounded theory. ${ }^{28}$ Transcriptions were coded according to step-wise processes entailing data immersion; distillation of key words/phrases; specification of concepts and categories; application and verification of a final coding structure; and identification of propositions (themes) and relationships evolving from the coded taxonomy. Open coding was carried out by two researchers (KW, RA) who worked independently to develop preliminary coding structures. They then met to compare and discuss the coding approach and category formation. Concurrent data coding and analysis allowed modifications to the topic guide for subsequent interview encounters to guide further understanding of developing categories. The first researcher (KW) is a pharmacist who previously provided inpatient care and supervised trainees in a multidisciplinary team setting. The RA was a graduate student in education with background in business and management organizational behaviours. The balance of the research team provided additional outside perspectives. They included an educationalist active in study of learning and assessment in the workplace [ED] and two medication education researchers who study how education supports learning from work and are physicians positioned within clinical teams themselves [FS, PT]. Throughout the data collection, the first author (KW) regularly revisited emergent categories and reviewed the evolving coding scheme with other co-investigators (ED, PT, FS) to confirm the axial coding, develop themes and consider their theoretical significance. This data-analytic process involved ongoing comparison between the interpretations of new findings with those made from previously collected data. Once the coding scheme had been refined, relationships among categories were explored to facilitate raising the analytic level from categorical to conceptual in order to enrich the data analysis. 
Ethics approval application processes was obtained from the University of British Columbia Behavioural Ethics Review Board (H18-00167) and the Vancouver Coastal Health Research Institute which has operational jurisdiction over Vancouver General Hospital (H18-02639).

\section{Results}

In the responses of multidisciplinary team members outlining communication and collaboration expectations for health professional learners training on their unit, we identified three main underlying perspectives related to practice. First, expectations could be broadly characterized by how trainees acquire and transmit patient information according to perceived best practices. Second, by how this information and other knowledge trainees possess and share is relevant to their own care. That is, when learners outside their own discipline communicate and collaborate in ways that impact their specific professional practice. Third, participant impressions of competent performance were influenced by teaching practices; a recognition that the team on this unit shares a responsibility to support the development of learners from many professions. Throughout the interviews, a distinction between communicator and collaborator roles was often not well delineated. When participants could not summon particular examples for pharmacy trainees, we summarized their viewpoints for any learner outside their discipline.

\section{General Expectations of Best Practice}

To understand how team members from different professions conceptualize communicator and collaborator competency roles for trainees outside their discipline, we contrasted with the behaviours they seek from trainees within their own profession.

Without exception, all participants invoked weekly interdisciplinary discharge rounds as the principal forum for interprofessional communication and outlined congruent preferences for the performance of trainees irrespective of discipline. For example, all trainees should be able to accurately convey pertinent information for this "rapid-fire" roundtable format.

"Short and sweet. Keeping it to the critical information." (Occupational Therapist 1)

We need to know, but we don't need to relay all detailed information in a time-critical environment such as in rounds." (Physiotherapist)

Concise and relevant presentation of patient information was also important to participants when a trainee from their own discipline was making recommendations to smaller groups or individuals. Demeanour and language adopted for these interactions was as meaningful as the subject matter they communicated.

"Mutual respect. I think if there's a tone... So, I always talk to my students about - is this the right time to be talking to the person? It this useful information for them to have or are you phrasing it in a way that's going to make them quite defensive?" (Nurse 2) 
"Communication is also the atmosphere they develop, right? That it's, you know, friendly and cooperative." (Physician 4)

Competent collaboration and communication were also signalled by these cooperative exchanges between team members and trainees from outside their own profession. Participants trusted trainees from outside their discipline when they clearly and confidently communicated patient issues.

"If someone offers a halting incomplete account, that is unclear... that sort of instantly increases your suspicion that like an error could happen and everything has to slow down." (Physician 3)

Timely follow-up through these personal interactions was valued. Although the team regularly met in structured environments to exchange patient assessments and plans, participants expected trainees from their own discipline to discuss issues directly with colleagues to ensure the continuity of information and care. Despite practical time constraints, most team members also preferred personal communication with trainees outside their discipline, even when the same information might be recorded elsewhere.

"I like when the [pharmacy] student will go up and ask, 'Hey, are they still having a fever? Are they still delirious? He could go to the chart and look and see, but he doesn't. He goes and talks to the nurses and I think that makes the relationship." (Nurse 2)

Although participants largely projected intra-professional performance expectations onto trainees outside their discipline, these perspectives were amended when considering patient and family encounters. In this instance, different participants did explicitly prioritize certain competencies for their own trainees that they did not expect from trainees outside their own discipline - demonstrating empathy (nurses), taking consent (occupational therapists), permitting patient agency (social worker). Physicians out rightly declared a belief that patient communication competencies required by their medical trainees was uniquely significant.

"We're all talking to the same sick people, but the agenda and the way that people perceive those conversations and the weight they put on them is quite different coming from the physicians." (Physician 3)

\section{Specific Expectations to Support my Practice}

Most participants contended that their expectations of best practices would apply to any trainee, irrespective of profession. Yet, how team members actually judged communicator and collaborator roles of pharmacy or other trainees outside their discipline were not entirely related to these performance standards.

Health professionals formed positive impressions of competencies when those trainees outside their discipline made discernible contributions to their own patient care. Sharing useful information was central to this judgement with minimal attention given to the specific communication or collaboration skillset these other trainees employed to do so. For example, in discharge rounds, participants were most interested in the patient assessments 
made by different professionals in order to make decisions for patients to safely transition from the unit (e.g. resolution of acute illnesses, mobility, diet, cognitive function, capabilities for self-care).

"The pharmacy student [at rounds] might say, 'actually we haven't changed that yet and the reason for this was'. They kind of tie up those pieces around meds." (Nurse 1)

In the participants' accounts of trainee contributions during rounds, they repeatedly referred to the content and not its delivery as a mark of competent communication and collaboration for trainees outside their discipline. This is in contrast to demeanour and language expectations they had outlined for the trainee within their own discipline. This predilection for function over form may explain why most of our participants did not scrutinize the written documentation of trainees outside their own disciplines. Only physicians singled out the utility of pharmacy or social work notes to communicate medication dosing and monitoring plans or expand on patient social histories. The information guided their medical decision-making, but they were not especially concerned with who the individual was that delivered it and how.

Expectations for readily available expertise is also reflected in the significance participants placed on trainees from other professions being visible and accessible on the unit. For example, pharmacist trainees were considered the resource related to medication information and viewed favourably when they could contribute to the team member's own knowledge and patient care.

"They come to participate in rounds. You see them in the nursing station all the time. So, I myself have gone up to them many times and said, 'oh hey. I think this patient might need a laxative, what would you suggest?' And I would see the nurses constantly going up to them and ask them things."

(Dietitian 1)

Such consistent descriptions illustrate how participants generally judge the performance of trainees outside their own discipline for their instrumental value and less so by the specific communicator and collaborator competencies the individual demonstrates.

\section{Perceived Commitment to a Teaching Practice}

As described above, participants underscored how decisions they must make for patient care are impacted by the information possessed and shared by trainees from other professions. In some ways, participants failed to differentiate between the skills and expertise evident in their colleagues - graduates in their respective professions often with several years' service - with their expectations for interprofessional trainee team function and fit. On the other hand, participants acknowledged the teaching nature of their unit and repeatedly expressed commitment to supporting all learners develop skills for team-based care. For example, while participants sought timely and accurate information from students, they ultimately recognized they were present on the unit to advance their training.

"It's a learning environment and they won't always have the answers." (Physician 1) 
Given such declarations of perceived commitment to a teaching practice, it is unsurprising many felt their own learners could benefit from interprofessional training input and how these "differing perspectives" could be useful in their development of communicator and collaborator roles.

"I will actually encourage residents [who] I am worried about their communication to sit with the social worker- to ask them to observe their running a family meeting and give feedback." (Physician 1)

If you are trying to provide the best care, you can't be the superman. You have to know everybody's specialty and admit that you could learn from this person. (Nurse 1)

Participants additionally relied upon their colleagues to offer impressions of student work and conduct when they were not present. In these ways, clinical supervisors could monitor performance while fostering autonomy in decision-making and care.

"I can't always be there to watch them or see how they collaborate with others. Who better to get feedback from than who she is directly talking to and on the receiving end?" (Dietitian 3)

However, a few participants opined that trainee performance might be adversely affected by additional scrutiny and could misperceive formative judgements as "high stakes". Instead, many described soliciting feedback with intention. That is, when participants sought interprofessional input it was from trusted colleagues who were additionally likely to have had repeated encounters with their trainee. The attributes of said consultants could be anticipated by proximity of daily work but less obviously by personal relationships cultivated between individuals on a team over time.

"I've known this physiotherapist for a long time and I know that when she says something, 99\% of the time she is right." (Physician 1)

Not all team members shared this extra-disciplinary information with their trainees, but did use colleagues' input as triangulating data to confirm or refute their own impressions, particularly for poorly performing trainees.

"Like post-call and they are just tired and that was a bad interaction, right. So those things [negative feedback] I just kind of listen and ignore unless I hear it more than once." (Physician 4)

"It reaffirms my assessment when other team members were giving me the similar feedback." (Dietitian 1)

As a supervisor, this collection (active or otherwise) of performance data to inform the assessment of their own trainee overshadowed any experiences as a team member offering formative feedback for trainees outside their discipline. Although they conveyed a commitment to the multidisciplinary teaching practice on their unit - "we are trying to be helpful"- few participants assumed any direct responsibility for shaping professional development of someone else's learner. Team members were careful to reiterate perceived feedback boundaries. For trainees outside their discipline, participants were generally comfortable offering their impressions of observed (or experienced) communication or 
collaboration, when asked. Some remained doubtful how meaningful their input would be for colleagues and their learners, particularly when it lacked specificity. Others would not want their colleague to place inordinate weight on their one perspective.

"I wonder how useful it is when my comments are just more general - "she seems nice" and those kind of things. (Dietitian 1)

"I guess the main thing would be that the preceptor would still probably know them best at the end of the day." (OT 1)

\section{Discussion}

This study set out to explore what communicator and collaborator competencies different health professionals expect from trainees outside their discipline. Team members identified and believed they adjudicated the performance of other trainees in relation to the standards of behaviours or 'benchmarks' they maintained for their own learner. In contrast with social identity theory which posits that traits of perceived fellow "in-group" members are preferentially favoured, our participants did not distinguish the best practices they expect of their own trainees from those of trainees outside their profession. ${ }^{23}$ Instead, we found participants seemed to judge other trainees according to how these roles were enacted in ways that specifically influenced their own abilities to provide patient care, with minimal real attention to the specific skillset the trainee demonstrated. We were unable to delineate meaningful differences in competency constructs across different disciplines as these were obscured by service-related expectations from trainees outside their profession, namely efficient and relevant information sharing.

Team expectations for trainee communication and collaboration were often shaped by the demanding conditions - high patient volume, varied and acute illnesses, complex discharges - under which they work and learn. Increasingly busy clinical learning environments erode opportunities for clinical supervisors to directly observe the activity of their health professional trainees. ${ }^{7,29}$ Indeed, these very shortcomings in part propel campaigns to enlist multiple perspectives, including those across health professions, as a means to increase validity and capacity for competency-based assessment and feedback. 2, 30 Our participants did form judgments of trainees outside their discipline based on direct observation (i.e. during rounds, on the unit, in personal encounters). However, these impressions were generally shaped by the timeliness and utility of the data provided and not necessarily the nuances of communication and collaboration they expect from their own trainee. In this way, team members viewed the student through the lens of a fellow healthcare provider and not someone on the unit for whom they have unique teaching responsibilities. It is unsurprising then that this outlook would be reflected in our data examining team member limited experiences offering formative feedback to trainees outside their discipline.

We interpreted mixed-sentiments among our participants who expressed some reluctance to provide feedback, especially for trainees with whom they had infrequent or isolated interactions. Despite sincere assertions of support for learner development on this clinical teaching unit, many participants doubted the utility of providing input that only amounted 
to a general impression. Unlike when supervising their own learner, team members were not actively considering the performance of other trainees for future evaluative purposes. They admitted to struggle recounting details of encounters with other trainees unless the communication or collaboration were markedly poor or outstanding. As such, potential performance-relevant information within said extremes of performance is unavailable to the learner. ${ }^{31}$

Conversely, team members were comfortable and accustomed to seeking multidisciplinary perspectives when they occupied the direct clinical supervisor role for a trainee in their own discipline. Our participants triangulated their own judgements with the views of others when preparing evaluations of their own trainee. Corroborating data from colleagues were considered especially useful, and therefore actively sought, when arriving at fail assessment decisions. Yet as Yarris asserts, additional stakeholder input may "replicate, elaborate, or complicate themes" identified by the principle supervisor. ${ }^{27}$ In other research, supervisors aggregated concordant global judgements made by clinical colleagues but de-emphasized and sometimes rejected reports from individual encounters. ${ }^{32}$ At least one participant in our study volunteered that they ignored singular accounts of poor trainee communication or collaboration. While our results did not expose disparate competency constructs across multidisciplinary member on this team, it remains unclear how supervisors attend to interprofessional feedback which conflicts with their own evaluation of their trainee. As such supervisory censure of inadequate performance may forego opportunities for competency development in important ways. ${ }^{33}$ Clinical supervisor discounting of conflicting outside perspectives, coupled with team member inability to remember directly observed performance of trainees outside their own profession invariably impacts the feasibility (and utility) of current multisource feedback models.

Multiple perspectives of trainee demonstrated skills and knowledge are meant to enrich competency development and learning. The collection of interprofessional viewpoints in particular, provides information on how trainees are contributing to and functioning in teambased care. However, learner benefits of such performance-relevant information may be lost among informal structures where this feedback is not routinely given directly to the trainee, nor to their direct supervisors, or when input is simply assimilated holistically by supervisors in end-of clerkship training reports. Instead, alternate models of "micro" educational alliances with different professionals might be cultivated through purposeful semistructured exercises focusing on specific aspects of competent practice (e.g. communication, collaboration, advocacy) and feasibly incorporated into usual care. ${ }^{34}$ It is clear from our study that characterizing mental models of shared competencies among professionals and a willingness to cross disciplinary boundaries to offer perspectives of trainee performance is ineffective if enabling systems are not in place.

Mastery of communication and collaboration in team-based care is a fundamentally social process. Interpersonal engagement is integral to developing abilities to express oneself and understand others and the means to exchange varied member knowledge and skills for shared decision-making. ${ }^{35}$ Our findings of how health professionals characterize trainee competence through observed and experienced reciprocal consultation and informationsharing underscore the significance of relationships in patient care and how learners make 
their mark in dynamic practice environments. Although they may overlook specific competency performance details of trainees outside their discipline, our participants promoted collaborative interdependence with them in patient care. Such inclusive attitudes and shared mentorship are aligned with the social component of an interprofessional learning environment. ${ }^{36}$ While members of this internal medicine unit espoused a teaching climate, the demands of patient care practically overshadowed regular provision of formative feedback of trainees outside their direct supervision. ${ }^{37}$ Overcoming fractured relationships between trainees and team members resulting from rare or intermittent encounters during short clerkships will be essential to capitalize on multiple perspectives in workplace learning.

This study is limited in a number of ways which may have affected our findings. We gathered data from a single inpatient-based medicine team in a large teaching hospital to ensure participants had wide and regular exposure to pharmacy and other trainees. Although participant expectations of trainee communication and collaborative roles on an acute medical unit are consistent with those reported for medical residents in pediatrics and critical care units, perspectives may be different among smaller teams, those delivering care on other units or in ambulatory-based settings. We generally framed the "other trainee" under consideration as a pharmacy student, but wider exploration of other learners (e.g. physician trainees) with whom more team members may regularly interact may yield different interprofessional role expectations. Although we followed theoretical sampling processes of grounded theory study, we were unable to interview all team members, particularly those who were part of the acute medicine network, but not physically colocated on the unit. It is possible that they may have provided additional views contributing to our presented themes. Our selection of communicator and collaborator roles may not represent all aspects of trainee performance that team-members might reasonably directly observe in practice. Nevertheless, these specific competencies practically appear as educational outcomes across a myriad of health professional training frameworks and augment the study's conceptual generalizability and transferability of our findings.

\section{Conclusion}

Multiple perspectives of trainee performance observed over time is advocated to enrich assessment in competency-based education. As collaborator and communicator competencies are especially pertinent to team functioning in patient care, trainee feedback from interprofessional members in the workplace can be valuable. We found what while declared multidisciplinary expectations of collaborator and communicator appeared uniform, team members actually formed impressions of trainees outside their own discipline according to how these competencies were exercised to advance their own professional practice decision-making or to support systems-level care. Team members expressed commitment to supporting trainees develop interprofessional practice competencies but we identified barriers to their ability to generate feedback that was specific or that actually reached the clinical supervisors and their trainees. Realizing the potential of team members as credible sources for trainee competency-based feedback and assessment requires practical approaches addressing the episodic, yet essential nature of these relationships. 


\section{References}

1. Van Der Vleuten CP, Schuwirth LW. Assessing professional competence: from methods to programmes. Med Educ. 2005; 39:309-317.

2. Lockyer J, Carraccio C, Chan M-K, Hart D, Smee S, Touchie C, Holmboe ES, Frank JR. Core principles of assessment in competency-based medical education. Med Teach. 2017; 39:609-616.

3. Ramani S, Krackov SK. Twelve tips for giving feedback effectively in the clinical environment. Med Teach. 2012; 34:787-791.

4. ten Cate O. Competency-Based Postgraduate Medical Education: Past, Present and Future. GMS J Med Educ. 2017; 34:1-6.

5. Stalmeijer RE, Dolmans DH, Snellen-Balendong HA, van Santen-Hoeufft M, Wolfhagen $\mathrm{IH}$, Scherpbier AJ.. Clinical teaching based on principles of cognitive apprenticeship: views of experienced clinical teachers. Acad Med. 2013; 88:861-865.

6. ledema R, Brownhill S, Haines M, Lancashire B, Shaw T, Street J. 'Hands on, hands off': a model of clinical supervision that recognises trainees' need for support and independence. Aust Health Rev. 2010; 34:286-291.

7. Watling C, LaDonna KA, Lingard L, Voyer S, Hatala R. 'Sometimes the work just needs to be done': socio-cultural influences on direct observation in medical training. Med Educ. 2016; 50:1054-1064.

8. Burford B, Morrow G, Rothwell C, Carter M, Illing J. Professionalism education should reflect reality: findings from three health professions. Med Educ. 2014;48:361-374.

9. Moonen-van Loon JM, Overeem K, Govaerts MJ, Verhoeven BH, van der Vleuten CP, Driessen EW. The reliability of multisource feedback in competency-based assessment programs: the effects of multiple occasions and assessor groups. Acad Med. 2015; 90:1093-1099.

10. CanMEDS 2015 Physician Competency Framework. Royal College of Physicians and Surgeons of Canada. Ottawa, ON; 2015.

11. Sonnenberg LK, Pritchard-Wiart L, Busari J. The resident physician as leader within the healthcare team: An exploratory inquiry into the perspectives of interprofessional clinicians. Leadersh Health Serv. 2018; 31:167-182.

12. Lattuca LR. Learning interdisciplinarity: Sociocultural perspectives on academic work. J High Educ. 2002; 73:711-739.

13. Lockyer J. Multisource feedback: can it meet criteria for good assessment? J Contin Educ Health Prof. 2013; 33:89-98.

14. Thistlethwaite JE, Forman D, Matthews LR, Rogers GD, Steketee C, Yassine T. Competencies and frameworks in interprofessional education: A comparative analysis. Acad Med.2014; 89:869-875. 
15. Canadian Interprofessional Health Collaborative (CIHC). A National Interprofessional Competency Framework. 2010. Vancouver, BC. Retrieved July 8, 2018. Available from: http://ipcontherun.ca/wp-content/uploads/2014/06/National-Framework.pdf.

16. Sharma N, Cui Y, Leighton JP, White JS. Team-based assessment of medical students in a clinical clerkship is feasible and acceptable. Med Teach. 2012;34:555-561.

17. Stalmeijer RE. Teaching in the clinical workplace: looking beyond the power of 'the one'. Perspect Med Educ. 2015; 4:103-104.

18. Schwind CJ, Williams RG, Boehler ML, Dunnington GL. Do individual attendings' postrotation performance ratings detect residents' clinical performance deficiencies? Acad Med. 2004; 79:453-457.

19. Hemmer PA, Dadekian GA, Terndrup C, Pangaro LN, Weisbrod AB, Corriere MD, Rodriguez R, Short P, Kelly WF. Regular formal evaluation sessions are effective as frame-of-reference training for faculty evaluators of clerkship medical students. J Gen Intern Med. 2015; 30:1313-1318.

20. Hauer KE, Cate Ot, Boscardin CK, lobst W, Holmboe ES, Chesluk B, Baron RB, O'Sullivan PS. Ensuring resident competence: a narrative review of the literature on group decision making to inform the work of clinical competency committees. Adv Health Sci Educ Theor Pract.2016; 8:156-164.

21. ten Cate $\mathrm{O}, \mathrm{Chen} \mathrm{CH}$. The parts, the sum and the whole-Evaluating students in teams. Med Teach.2016; 38:639-641.

22. Stone PC, Kagotani K. The wisdom of the multitude: diversity versus size. Applications of Formal Philosophy. Springer; p. 55-71.

23. Burford B. Group processes in medical education: learning from social identity theory. Med Educ. 2012;46:143-152.

24. Currie G, White L. Inter-professional barriers and knowledge brokering in an organizational context: the case of healthcare. Organ Stud. 2012; 33:1333-1361.

25. Whitehead CR, Kuper A, Hodges B, Ellaway R. Conceptual and practical challenges in the assessment of physician competencies. Med Teach. 2015; 37:245-251.

26. Thompson BM, Gonzalo JD, Levine RE. The power of the written word: team assessment of behaviour. Med Educ. 2016; 50:706-708.

27. Yarris LM. Defining trainee competence: value is in the eye of the stakeholder. Acad Med. 2019; 94:760-762.

28. Charmaz K. 2006. Constructing grounded theory: A practical guide through qualitative analysis. Sage Publications: London, UK.

29. Cleland J, Durning SJ. Education and service: how theories can help in understanding tensions. Med Educ. 2019; 53:42-55.

30. Harris P, Bhanji F, Topps M, Ross S, Lieberman S, Frank JR, Snell L, Sherbino J, Collaborators I. Evolving concepts of assessment in a competency-based world. Med Teach. 2-17; 39:603-608. 
31. van der Leeuw RM, Teunissen PW, van der Vleuten CP. Broadening the scope of feedback to promote its relevance to workplace learning. Acad Med. 2018; 93:556-559.

32. Castanelli DJ, Weller JM, Molloy E, Bearman M. Shadow systems in assessment: how supervisors make progress decisions in practice. Med Educ. 2020;25:131-147.

33. Patel M, Agius S, Wilkinson J, Patel L, Baker P. Value of supervised learning events in predicting doctors in difficulty. Med Educ. 2016; 50:746-756.

34. Min AA, Spear-Ellinwood K, Berman M, Nisson P, Rhodes SM. Social worker assessment of bad news delivery by emergency medicine residents: a novel direct-observation milestone assessment. Intern Emerg Med. 2016; 11:843-852.

35. Mao AT, Woolley AW. Teamwork in health care: maximizing collective intelligence via inclusive collaboration and open communication. AMA J Ethics. 2016;18:933-940.

36. Gruppen LD, Irby DM, Durning SJ, Maggio LA. Conceptualizing learning environments in the health professions. Acad Med. 2019; 94:969-974.

37. Schein EH. Organizational culture. Am Psychol.1990; 45:109-19. 



\section{CHAPTER 5}

Pharmacist Trainees' Narrow Scope of Interprofessional Collaboration and Communication in Hospital Practice

Submitted 


\begin{abstract}
Early curricular exposure to interprofessional education (IPE) is intended to acclimatize health professional trainees to shared-care in the practice settings they will ultimately join. However, IPE activities typically reside outside actual organizational and social systems in which interprofessional care is delivered. We aimed to explore how pharmacist trainees experience collaborator and communicator competency roles during team-based workplace-based learning. Participants maintained written diaries reflecting on interprofessional collaboration and communication during an eight-week hospital clerkship. Diary entries and transcripts from semi-structured follow-up interviews were analyzed from the social constructivist perspective using reflective thematic analysis. Participant accounts of on-ward activities represented most collaborator and communicator roles outlined in pharmacy and interprofessional competency frameworks, but were predominantly between the pharmacist trainee and physicians. Pharmacist trainees did not routinely engage with other health professions on a daily basis. Additionally, reported encounters with other team members were typically information exchanges and not episodes of authentic interdependent or shared care. Interactions were almost completely devoid of perceived interpersonal or role conflict. These findings offer insight into how pharmacist trainees perceive and develop competencies for team-based care. Further work is required to understand how such limited scope of interprofessional communication and collaboration might ultimately impair quality patient care.
\end{abstract}




\section{Background}

Contemporary healthcare is fundamentally a team-based enterprise. As populations age, chronic diseases become more prevalent, and treatments further specialized, patient care is increasingly complex requiring the expertise of diverse health professionals. Indeed, evidence demonstrates that effective team-based care can improve patient satisfaction and contribute to positive clinical outcomes. ${ }^{1}$ It is unsurprising then that health professional training programs have integrated interprofessional education (IPE) into their curriculum, whereby students from two or more disciplines, 'learn about, from, and with each other'.2 Early exposure to extra-disciplinary knowledge and skills is meant to promote mutual understanding of one another's scopes of practice and acclimatize trainees to shared-care in the settings they will ultimately join. Practically, there are limits to how IPE can adequately prepare trainees to work in teams. Activities are often episodic, conducted in campusenvironments, facilitated by non-clinical academic faculty, and often don't go beyond simulating decision-making and care with other learners., ${ }^{3,4}$ IPE programming therefore typically resides outside the actual organizational and social systems in which interprofessional care is delivered. We know little about health professional trainee interprofessional competency development and performance when they join established teams in situ during workplace-based learning.

The competencies necessary for health professionals and trainees to optimize shared-care are outlined in a number of interprofessional frameworks. ${ }^{5}$ Expected cognitive and affective abilities include applying one's expertise to plan and implement patient care, exchanging professional knowledge, acknowledging differences, and maintaining a climate of mutual respect, to name a few. Skilled communication and collaboration are essential to all elements of team functioning. Interprofessional communication and collaboration competencies additionally reflect descriptions found in discipline-specific educational outcomes. For example, graduating dietitians, nurses, pharmacists, physicians, and physiotherapists are among those trainees who must have demonstrated proficient written and verbal communication and collaborative relationships by the end of their program. ${ }^{6-8}$ Yet how these interprofessional competencies are explicitly enacted on teams in workplacebased learning is unclear. When the content of third-year medical student written reflections on work relevant to patient care during hospital and community placements were mapped to the CanMEDS physician competency framework, communicator roles were represented in $40 \%$ of records and collaborator roles made up $4 \% .{ }^{9}$ In a similar study of onward activity logs made by medical students during a 16-week internal medicine rotation, $42 \%$ of performed activities were classified as communication and $7 \%$ as collaboration. ${ }^{10}$ In these studies, information about whether the learners themselves detected omissions in practice is not reported.

However, other research has reported trainee perspectives on practicing interprofessional competencies during clerkship. When asked to specifically consider communication and collaboration opportunities, learners across different disciplines did perceive inadequacies. Physiotherapy students described insufficient contact and direct communication with other professionals outside of formal team encounters during inpatient clinical placements. ${ }^{11}$ Nursing students also indicated how interprofessional collaboration within their assigned 
team was hindered by ineffective communication and conflict avoidance. ${ }^{12}$ Similarly, pediatric medical residents felt aspects of the collaborator competency role were absent from their training. They were missing faculty role modelling and strategies appropriate for managing conflict, especially those arising on nights and weekends when they were working independently. ${ }^{13}$ Despite serving key functions in health professional education and patient care, trainees may be facing barriers to exercising all dimensions of communication and collaboration competencies in team-based practice settings.

Actual and perceived gaps in interprofessional competency development have a number of implications for health professional education and care delivery. From the trainee's immediate perspective, deficient learning opportunities could interfere with self-regulated learning. If trainees do not recognize and ascribe value in a learning task, they will not set goals or plan strategies to seek out or accomplish it. ${ }^{14}$ In contexts where the potential learning experience is lacking, the trainee can neither perform, nor reflect upon their behaviour. ${ }^{15}$ These missed opportunities for practice can reverberate into the future when graduates first assume positions on teams. Novice professionals admit to feeling ill equipped for this transition, specifically their abilities to deal with criticism, negotiate conflict and communicate succinctly with colleagues. ${ }^{16,17}$ By extension, inability of teams to work effectively has adverse effects on healthcare. Communication failures and incomplete use of available expertise is attributed to increased risk of medical harm. ${ }^{18}$ Gaining insight into trainee opportunities and engagement with interprofessional competency roles during clerkship experiences can inform how to mitigate these short and long term consequences on learners and ultimately patients. In this study, we used longitudinal diaries and interviews to explore how trainees experience collaborator and communicator competency roles in team-based workplace-based learning.

\section{Methods}

We adopted a social constructivist stance to explore trainee descriptions of performed collaborator and communicator competency roles and associated feedback within the framework of a longitudinal workplace-based study. In this study, we position the trainee at the center of the meaning-making experience within the social context of team-based patient care.

Setting and Study Population

The Faculty of Pharmaceutical Sciences at the University of British Columbia (UBC) enrolls one of the largest undergraduate pharmacy student bodies in Canada ( $N=224$ per class). In Canada, pharmacist trainee educational outcomes are modelled by the Association of Faculties of Pharmacy (AFPC) after the CanMEDS physician competency framework with the aim to graduate not Medical, but Medication Experts. ${ }^{19}$ The full-time experiential training component of the curriculum totals 42-weeks and the majority occurs in the fourth and final training year. One of the mandatory clerkship courses fourth year students complete is the 8-week inpatient Advanced Pharmacy Practice Experience conducted in a hospital teambased patient care setting under the supervision of a pharmacist preceptor. This study population was chosen as they have completed all components of the campus-based IPE 
curriculum. Using a volunteer sampling strategy, we recruited 25 fourth year pharmacist trainees completing this clerkship course to participate in the study during the 2019/2020 academic year (August 2019 to April 2020).

Data Collection

\section{Longitudinal Diaries}

Participants maintained written workplace-activity diaries during the inpatient clerkship course. Diary methods aim to capture time-sensitive and context-dependent participant data. ${ }^{20}$ Through their written dairies, it is possible to examine learner perspectives as documented proximal to events and is particularly relevant in longitudinal research designs. ${ }^{21}$ Participant recording or "journaling" can additionally stimulate affective learning related to work and encounters in team-based care. ${ }^{22}$

Specific question prompts were utilized to illicit examples of communication and collaboration activities: What are the specific opportunities you have had to develop your skills as a communicator? As a collaborator? These could be encounters with patients or family members or interactions with other care providers. Please give one or more examples that stood out for you in the clerkship so far. Diary prompts also stimulated pharmacist trainee reflection on their competency development at personal, professional, and interprofessional levels, including any feedback they received: What feedback have you received (formal or informal) on your performance as a communicator so far? As a collaborator? At pre-determined time intervals (clerkship weeks 2, week 6, and week 8) participants submitted diary records through a secure web-based platform. A research assistant (RA) managed all data and followed up on any missing data. All diary records were organized by individual participant and de-identified before research team review. Authors, workplace-based clerkship supervisors, and program coordinators maintained no knowledge of specific pharmacist trainee study enrolment.

\section{Interviews}

The RA conducted semi-structured interviews with participants following the conclusion of the inpatient clerkship course to gain further understanding of student experiences and views. The discussion topic guide was informed by the research questions and by findings taking shape in the iterative analysis of all diary data. Questions further explored the nature of encounters with non-pharmacist team members, and any perceived differences in participant expectations for interprofessional communication and collaboration, and their experiences in actual hospital practice. Excerpts from an individual's submitted diary records were also incorporated as participant-specific probes. These interviews were audiorecorded and professionally transcribed verbatim. Returned transcripts were checked against original audio-recordings by the RA and finalized. Like the diary records, interview participant identity was blinded to the rest of the research team. 


\section{Data Analysis}

First, a directed coding approach was applied to participant diary content of perceived collaborator and competency performance. The specific recorded workplace-based activity was coded and mapped to relevant communicator and collaborator competency descriptions in both national pharmacy-specific and interprofessional competency frameworks (Appendix). ${ }^{23,24}$ Mixed author pairs (KW, GP, JY) independently coded and mapped participant diary data. Following comparison of these initial allocations, differences in researcher selections were identified and resolved through consensus discussion. The Appendix figure illustrates this process for communicator and collaborator competency rolerelated diary entries for one participant across their 8-week clerkship.

Second, diary and interview data were analyzed using reflexive thematic analysis to identify and describe patterns across our data. ${ }^{25,26}$ Data familiarization (multiple readings of transcriptions and review of audio-recordings and coding memos) was followed by preliminary open coding (focusing on surface meanings). Codes were further refined and clusters of coded data sharing meaning were organized into developing themes. Themes were further reviewed before finalization with feedback from colleagues in seminar forums. Coding was initiated by the first author (KW) and reviewed by additional researchers (GP, JY). Theme development was led by the first author in consultation with senior authors (ED, FS, PT). Data collection (interviews) and analysis (interviews and diaries) occurred iteratively. The research team members held regular discussions during these processes to consider and debate themes and plan for subsequent interviews accordingly.

Ethics approval was granted by the UBC Behavioural Research Ethics Board. Given the longitudinal nature of the research project, informed consent was obtained during recruitment for the initial diary data collection phase and again prior to the semi-structured interviews. During the academic year, three trainees enrolled in the study withdrew from the clerkship courses and did not make any diary entries. Nine of the remaining twenty-two participants were unable to submit the full complement of diary records as they were removed from their inpatient clerkship course prior to its conclusion due to COVID-19 public health emergency. We analyzed what records they were able to submit. A total of 96 diary entries were included in our data set and thirteen participants completed the follow-up interview.

\section{Results}

\section{Interprofessional Collaboration and Communication in Practice}

Pharmacist trainees recorded activities during the eight-week clerkship which could be indexed to most of the collaborator and communicator competencies outlined in both the pharmacy and interprofessional competency frameworks. In their diary entries, pharmacy trainees did not always distinguish between collaborator and communicator competency roles in their recorded experiences. We therefore mapped the responses to discrete diary prompts across both collaboration and communication descriptions in the competency frameworks (Appendix). ${ }^{23,24}$ 
Working with others to provide safe, effective, and efficient healthcare was evident in trainee diary entries, but recorded interprofessional encounters were overwhelmingly between the pharmacy trainee and a physician. Instances communicating intra- and interprofessional patient handover (described in the pharmacist collaborator competency role) were largely absent from clerkship experiences. We were also unable to identify documented instances consistent interprofessional conflict and its resolution (as outlined for collaborative care in the interprofessional competency framework), although pharmacist trainees offered frequent accounts of correcting physician prescriptions.

Participant interview permitted elaboration and further exploration of pharmacy trainee experiences documented in diary entries. Analysis of diary and interview data yielded three main themes of how interprofessional collaboration and communication are enacted in hospital practice:

\section{Theme 1 - Limited Interaction with Different Health Professionals}

The diaries clearly revealed the routine daily work of pharmacist trainees requiring physician contact. Episodes chronicling prescription clarifications, dosing confirmations, and therapeutic recommendations with various physicians were repeated by all participants throughout the eight weeks. Communication or collaboration with nurses were not widely represented in the diaries, but participants readily described these interactions in the interviews. Pharmacy trainees responded to nurse inquiries about drug formulations or medication effects and often sought the nurse's assessment of patient response to therapy, as well as overall disposition.

"The nurses were very helpful. I asked them a lot of questions regarding recent vitals that weren't updated or changes in medications. So, I would have daily conversations with the nurses."

(G18 interview)

"I was asking nurses, because they're the most in touch with the patients and exactly how they're doing - like if they're declining or if they're improving, if they have any new concerns that they need addressed... things like that." (Y14 interview)

Unlike physicians and nurses, pharmacist trainees did not regularly engage members of other health professions during the hospital clerkship. Most trainees did attend multidisciplinary rounds with their clinical supervisor where they observed health professionals "coming together to make plans for patients in a holistic way". A small number of participants described shadowing opportunities whereby they followed another team member (e.g. social worker, occupational or physiotherapist) for half-day to learn more about their practice. However, pharmacist trainees worked infrequently with these health professionals in an interdependent way. 


\section{Chapter 5}

\section{Theme 2- Opportunities for Authentic Shared Care}

Pharmacist trainee diaries were replete with episodes of knowledge transfer with physicians and our interviews yielded many examples of information exchange with nurses. Sharing medication or patient data facilitated each professional's own provision of care, but on its own such consultation would not constitute true shared decision-making. However, in our interviews participants recounted a number of specific instances where they cooperated with physicians to provide joint care.

"A medical resident had the same patient and we collaborated to make decisions. I had some medication recommendations that we were able to implement through discussion of the patient's goals."

(J11 Interview)

Participants also reported collaborative experiences with other health professionals. Although pharmacist trainees confirmed these encounters were few, these collective efforts to solve specific patient problems resonated.

"There was a patient getting multiple hypoglyemic episodes and I wanted to figure out with the dietitian how they're snacking and eating so we could make a recommendation to the doctor." (G18 interview)

"I had an extensive interaction with one of the social workers because we were trying to figure something out for one of the patients. And then they couldn't afford the medication, so we kind of went to work with them to kind of figure out options. There was a lot of collaborating - which was a good experience." (U30 interview)

Even informal (and ostensibly passive) job shadowing yielded meaningful opportunities for pharmacist trainees to form relationships and further understand how each other's roles augment patient care.

"The physiotherapist said I was one of the only pharmacy students to ever ask to shadow them and see what they do and how they are part of the patient's health care team." (L28 diary)

"[The social worker] interacted with the patient from a different perspective for totally different concerns that, you know, I had no knowledge about. But, it was just really being able to see his empathy skills and, you know, really work with the patient to find the best outcome for themselves or their family member at that time. So, he was really listening and, you know, provided me the insight that you really have to collaborate with the patient." (K60 interview)

"I was recommending insulin, but the patient's movement and mobility was very important for the recommendation I was going to make. So, you know I thought to consult the OT because I had a chance to chat with her the prior week about what she really does here." (G18 interview) 
Theme 3 - Adequate Interprofessional Competency Development Perceived

Pharmacist trainees were largely unsurprised about the nature of work with physicians in hospital practice. Participants were expecting to communicate their ideas "clearly" and "concisely" with adequate information supporting their medication recommendations to different prescribers in writing and in person (individually or in rounds). They recognized the need to convey timely, accurate drug information to collaborate with others. Most pharmacist trainees commented how their communication skills improved through recurrent deliberate practice with physicians.

"I was saying, well, the first drug has this problem and then I was going to say some other things that were problems with it. And my preceptor was like, "Stop. If the first reason is a good enough reason, you don't need to give others." (V22 Interview)

"My preceptor has indicated that my interactions with [physicians] are generally good, but occasionally I would benefit from rearranging the information presented to highlight the most pertinent findings." (S26 diary)

While conceptually aware of health professional roles, a number of participants found what they practically observed in multidisciplinary rounds noteworthy - active listening and respectful exchanges between individuals that role modelled team approach to care. Participants also explained initiating interactions with non-physician team members with minimal direction by their clinical supervisor. Although ability to readily access other health professionals varied by clerkship site, pharmacist trainees were sufficiently acquainted with other disciplinary roles to seek their expertise for patient care, albeit sporadically.

While generally satisfied with the opportunities to practice interprofessional communication and collaboration competencies, pharmacist trainees were rarely faced with interdisciplinary conflict and gave no indication this was an oversight in their competency development. The authors liberally mapped episodes where pharmacist trainees resolved medication errors or discrepancies with physicians as interprofessional conflict management, although participants themselves never labelled these encounters as such. They anticipated potential resistance from prescribers when pursuing modification or correction of drug therapy.

"I wanted to deescalate the antibiotic therapy for a specific patient once the culture sensitivity came back, and one of the problems I ran into was the doctor went like, well it's worked so far and they have like two days left in the therapy, so they just kept them on it. So that's kind of frustrating, but I don't think it's a point that I really should be spending so much time fighting over." (V22 Interview)

However, in most instances described by participants, medication recommendations were invariably accepted by physicians with minimal disagreement. 


\section{Discussion}

In this study, we sought to understand how pharmacist trainees enact specific interprofessional competencies in workplace-based learning settings situated within hospitals. Characterization of pharmacist trainee written reflections of on-ward clerkship activity encompass the majority of the communicator and collaborator roles outlined in pharmacy and interprofessional competency frameworks. ${ }^{9,10}$ Diary entries offer insight into workplace affordances for interprofessional care and competency development, as well as ways trainees accomplish work with others. Social constructivism posits that knowledge develops from one's interactions with the social world and therefore meaning is built by matching new ideas and experiences against existing knowledge. ${ }^{27}$ The episodes of daily care pharmacist trainees chose as representative examples of communication and collaboration additionally reveal their evolving perceptions of roles in team-based practice. Taken together with interview data, reports from these hospital clerkship experiences portray interprofessional competencies developing through largely transactional exchanges with few mixed disciplinary members and almost completely devoid of interpersonal or role conflict. Pharmacist trainees practiced most forms of interprofessional communication and collaboration with the physicians.

Physician-centric interprofessional care exercised by pharmacist trainees may be anticipated given the dynamic inherent to their respective scopes of practice. In their most fundamental role, pharmacists must ensure the appropriate and correctly dosed medication reaches the intended patient, thereby necessitating regular contact with the prescribers. Across these inpatient settings, pharmacist trainees were included in multidisciplinary and ward rounds, but spent relatively more time engaged with individual physicians separately, in-person or by telephone. It was also evident in trainee accounts how their pharmacist supervisors invested time coaching them for these varied physician encounters - use persuasive language, chose compelling data, present in a logical fashion, be succinct. Emphasis and apparent attention to careful communication with physician members of the healthcare team is not necessarily unique to pharmacists and their trainees. While interprofessional team members may dispute the physician's role as default leader in patient case management, in many ways they are also structurally reliant upon the prescriber to authorize aspects of their discipline-specific care plans. ${ }^{28,29}$ In fact, physician performance is often paradoxically judged by how "consensually collaborative" they are in working with others while at the same time maintaining directive authority. ${ }^{30,31}$ Patient care decisionmaking hierarchies in hospital settings inevitably drive interprofessional encounters for most team members (and their trainees) towards physicians.

Given how our participants often communicated with physicians using deliberate and premeditated scripts, it is remarkable that pharmacist trainees did not articulate conflict experiences nor acknowledge potential conflict-avoidance tactics with prescribers as such. Furthermore, they denied conflict experiences with team members of any other disciplines. Unlike findings among other health professional trainees in hospital practice, pharmacist trainees did not address lack of interprofessional conflict management practice as a gap in their learning experience. ${ }^{11-13}$ Interprofessional disputes stem in part from differences pertaining to patient care and can manifest in tense atmospheres, rude exchanges, and 
disruptive conduct. ${ }^{32}$ It is highly unlikely that these teams across diverse patient care units in different hospitals and geographic regions were devoid of conflict during the study period. Instead, pharmacist trainees' failure to discern conflict may reflect a superficial aspect to their reported interprofessional work. It is conceivably easier to elude contentious situations when there is minimal interaction with varied professionals or when cooperation is represented as simple information exchange. In contrast, interdependent patient care often requires deliberation of opposing perspectives or clarification of misunderstandings which when resolved constructively, can lead to improved decision-making. Another consideration is the role the supervisors and even other healthcare team members play to concertedly shield trainees from potentially unpleasant discord. ${ }^{13}$ Our results clearly signal the need for future work uncovering trainee experiences and management of interprofessional conflict in clinical learning environments.

Pharmacist trainees were largely satisfied with the opportunities afforded in hospital clerkships to develop interprofessional communication and collaboration. They anticipated all medication management roles they enacted with physicians. These expectations were further reinforced by supervisor predisposition for performance feedback oriented towards physician encounters. Apart from nurses, non-physician team member interactions were markedly absent in daily care, conceivably limiting the true scope of enacted interprofessional communication and collaboration - yet pharmacist trainees noted no insufficiency. University campus-based IPE programming assuredly strives to create relevant mixed-disciplinary groups according to activity, but what often forms are arguably manufactured teams of convenience. So is it that pharmacist trainees possess narrow expectations of interprofessional competency development readily met through physician (many) and nurse (relatively fewer) encounters or did they experience an authentic hospital pharmacy practice model? The nature of specific disciplinary expertise and scope of practice invariably yields more routine proximal working relationships among certain professionals. However, broader orbits of team cooperation exist whereby nurses and other multidisciplinary members engage in rich, patient-focused problem-solving and care planning, separate from physicians. ${ }^{33}$ The pharmacist's relative position across such decision-making networks in hospital teams has not been well-examined, but our data suggests pharmacist trainees are more likely to find themselves conducting interprofessional work with the physicians. As we earlier asserted, substantial elements of all interprofessional care ultimately invokes a physician, but the medications themselves (e.g. the essential provision of appropriate drug choices to patients in correct doses in a timely fashion) may be a distinguishing apparatus for pharmacists. While this gravitation towards prescriber contact is pragmatic to fulfilling daily tasks, it is unclear how this might compromise the full dimension of pharmacist training and ultimately provision of patient care.

Participatory practice theory posits that learning in the clinical environment hinges on both workplace affordances and active learner engagement. ${ }^{34}$ Based on participants' reports, they appear to possess adequate interprofessional role awareness as indoctrinated by their IPE curriculum. Although far less frequent or ritualized, pharmacist trainees appropriately sought out other non-physician team members for disciplinary expertise to incorporate into their medication-oriented care decisions. While encouraging, these interactions represent the most basic form of interprofessional collaboration, lacking negotiated agreement or 
shared responsibility. ${ }^{35}$ The paucity of authentic shared-care described during these hospital clerkships may not be readily overcome through greater pharmacist trainee agency. Social and material conditions beyond the learners' control are influential factors on competency development in these workplaces. Availability of physical space where pharmacists and other team members naturally converge performing daily tasks will shape how interprofessional care is learned and practiced. ${ }^{36}$ Similarly, adaptive interprofessional communication and collaboration is necessary when team members are not co-located or visibly apparent on the patient care unit. ${ }^{37}$ Adverse effects of these tangible elements are further exacerbated by certain social and cultural orientations in the workplace. ${ }^{36}$ Healthcare teams and individual members can be sincerely kind and welcoming - while concurrently assigning trainees to marginal status. ${ }^{38}$ For example, pharmacy trainees at all hospital units in our study were invited to rounds, but often simply as observers. Such exclusion is not active censorship, but is possibly part of how teams adapt (or don't) to the continuous incursion of health professional trainees onto their units. We know that relationships facilitating cohesive work among team members are built over time. ${ }^{18}$ How much true interdependent care with varied professionals should we expect of trainees joining teams for many weeks and not many months? ${ }^{39}$

Relatively speaking, perhaps the most easily adjusted variable in the clinical learning environment is the supervisor. Clinical teachers who deliberately assign work requiring interdisciplinary consultation and designate responsibility to trainees for this patient care promote peripheral participation. ${ }^{40}$ A supervisor's pre-existing interprofessional connections with other team members facilitates such trainee integration. ${ }^{41}$ Interprofessional care is further role modelled through witnessed interdisciplinary encounters, not only shaping trainee behaviours, but also attitudes and identity. ${ }^{39}$ The supervisor is an influential guide for trainees connecting formalized expectations of interprofessional collaboration and communication in the curriculum with what happens on a team in actual practice. With them, trainees can reflect on perceived discrepancies and consider how they might effectively build care networks in future multidisciplinary contexts. Further work is required to understand how the relationship between teacher and trainee factors into co-regulated learning and interprofessional competency development as well as collaborative practices trainees adopt in subsequent team settings.

Potential limitations of our study should be noted. Although competency descriptions appear on the instruments used for in-training evaluation reports (ITERS), we did not expressly reorient participants to these definitions as part of the study. Pharmacist trainees were prompted to record episodes of care that they interpreted to be examples of communication or collaboration and therefore other activity that might have matched competency framework content was not captured. However, we contend that trainees embedded in clinical learning environments will develop understanding outside formal curricular definitions of competencies. ${ }^{42}$ Pharmacy trainees documented clerkship experiences in study diaries at three pre-determined intervals during the eight-week clerkship and some follow-up interviews occurred several weeks following its conclusion. While this time lag could have compromised accurate participant recall of clerkship experiences, the diary data was in fact replete with concrete examples and stimulated rich responses when embedded as participant-specific interview prompts. Clerkship experiences 
under study took place in different specialty units at teaching and non-teaching hospitals in urban and non-urban regions servicing acute and non-acute patient care. We did not compare participant experiences according to hospital clerkship site. Given the contextspecificity of learning, subsequent study of organizational contexts of workplace-based settings and how they shape interprofessional competency development is worthy of ongoing inquiry.

\section{Conclusion}

Our study findings offer insight into how pharmacist trainees perceive interprofessional care and are developing competencies for team-based practice. Unlike simulated campus-based interprofessional education activities, described daily work of pharmacist trainees in hospital clinical learning environments involves few non-physician, non-nursing multidisciplinary members. Professional knowledge and patient information is freely exchanged, but evidence of actual shared decision-making responsibility and any associated conflict is lacking. It remains unclear if these experiences simply represent where pharmacist trainees are in their practice trajectory towards independent care or if retention of such limited scope of interprofessional communication and collaboration is retained by pharmacists in teams and ultimately impairs quality patient care. 


\section{References}

1. Schmutz JB, Meier LL, Manser T. How effective is teamwork really? The relationship between teamwork and performance in healthcare teams: a systematic review and meta-analysis. BMJ Open. 2019;9:e028280.

2. World Health Organization. Framework for action on interprofessional education and collaborative practice. Geneva: World Health Organization; 2010.

3. Fox L, Onders R, Hermansen-Kobulnicky CJ, et al. Teaching interprofessional teamwork skills to health professional students: A scoping review. J Interprof Care. 2018;32:127135.

4. Joynes VC. Defining and understanding the relationship between professional identity and interprofessional responsibility: implications for educating health and social care students. Adv Health Sci Educ Theory Pract. 2018;23:133-149.

5. Thistlethwaite JE, Forman D, Matthews LR, Rogers GD, Steketee C, Yassine T. Competencies and frameworks in interprofessional education: A comparative analysis. Acad Med. 2014;89:869-875.

6. Tong B, St John M, Li E, Wilbur K. Could interprofessional education assessment in workplace learning be streamlined? J Interprof Educ Pract. 2020:19; 100321.

7. Verma S, Paterson M, Medves J. Core competencies for health care professionals: what medicine, nursing, occupational therapy, and physiotherapy share. J Allied Health. 2006;35:109-115.

8. Verma S, Broers T, Paterson M, Schroder C, Medves JM, Morrison C. Core competencies: The next generation: Comparison of a common framework for multiple professions. J Allied Health. 2009;38:47-53.

9. Bennett D, McCarthy M, O'Flynn S, Kelly M. In the eye of the beholder: student perspectives on professional roles in practice. Med Educ. 2013;47:397-407.

10. Bugaj TJ, Schmid C, Koechel A, et al. Shedding light into the black box: A prospective longitudinal study identifying the CanMEDS roles of final year medical students' onward activities. Med Teach. 2017;39:883-890.

11. Robson $M$, Kitchen SS. Exploring physiotherapy students' experiences of interprofessional collaboration in the clinical setting: A critical incident study. J Interprof Care. 2007;21:95-109.

12. Salfi J, Mohaupt J, Patterson C, Allen D. Reality check: Are we truly preparing our students for interprofessional collaborative practice? Can J Nurs Res. 2015;47:41-61.

13. Berger E, Chan M-K, Kuper A, et al. The CanMEDS role of Collaborator: How is it taught and assessed according to faculty and residents? Paediatr Child Health. 2012;17:557560.

14. Berkhout JJ, Helmich E, Teunissen PW, van den Berg JW, van der Vleuten CP, Jaarsma ADC. Exploring the factors influencing clinical students' self-regulated learning. Med Educ. 2015;49:589-600. 
15. van Houten-Schat MA, Berkhout JJ, van Dijk N, Endedijk MD, Jaarsma ADC, Diemers AD. Self-regulated learning in the clinical context: a systematic review. Med Educ. 2018;52:1008-1015.

16. Hezaveh MS, Rafii F, Seyedfatemi N. Novice nurses' experiences of unpreparedness at the beginning of the work. Glob J Health Sci. 2014;6:215.

17. Sturman N, Tan Z, Turner J. "A steep learning curve": junior doctor perspectives on the transition from medical student to the health-care workplace. BMC Med Educ. 2017;17:92.

18. Rosen MA, DiazGranados D, Dietz AS, et al. Teamwork in healthcare: Key discoveries enabling safer, high-quality care. Am Psychol. 2018;73:433.

19. CanMEDS 2015. Physician Competency Framework. Royal College of Physicians and Surgeons of Canada. Ottawa, ON; 2015.

20. Hyers LL. Diary Methods: Understanding Qualitative Research. Oxford University Press; 2017.

21. Ciere Y, Jaarsma D, Visser A, Sanderman R, Snippe E, Fleer J. Studying learning in the healthcare setting: the potential of quantitative diary methods. Perspect Med Educ. 2015;4:203-207.

22. Rogers GD, Thistlethwaite JE, Anderson ES, et al. International consensus statement on the assessment of interprofessional learning outcomes. Med Teach. 2017;39:347-359.

23. Canadian Interprofessional Health Collaborative $(\mathrm{ClHC})$. A National Interprofessional Competency Framework. Vancouver, BC; 2010.

24. Association of Faculties of Pharmacy in Canada (AFPC). Educational Outcomes for First Professional Degree Programs in Pharmacy in Canada. Ottawa, ON; 2017.

25. Braun V, Clarke V. Using thematic analysis in psychology. Qual Res Psychol. 2006;3:77101.

26. Braun V, Clarke V. Reflecting on reflexive thematic analysis. Qual Res Sport Exerc Health. 2019;11:589-597.

27. Mann KV. Theoretical perspectives in medical education: past experience and future possibilities. Med Educ. 2011;45:60-68.

28. Sonnenberg LK, Pritchard-Wiart L, Busari J. The resident physician as leader within the healthcare team: An exploratory inquiry into the perspectives of interprofessional clinicians. Leadersh Health Serv. 2018;31:167-182.

29. Bourgeault IL, Mulvale G. Collaborative health care teams in Canada and the USA: Confronting the structural embeddedness of medical dominance. Health Sociol Rev. 2006;15:481-495.

30. Renting N, Dornan T, Gans RO, Borleffs JC, Cohen-Schotanus J, Jaarsma ADC. What supervisors say in their feedback: construction of CanMEDS roles in workplace settings. Adv Health Sci Educ Theory Pract. 2016;21:375-387. 
31. Lockyer J. Multisource feedback in the assessment of physician competencies. J Contin Educ Health Prof. 2003;23:4-12.

32. Bochatay N, Bajwa NM, Cullati S, et al. A multilevel analysis of professional conflicts in health care teams: insight for future training. Acad Med. 2017;92:S84-S92.

33. Zwarenstein M, Rice K, Gotlib-Conn L, Kenaszchuk C, Reeves S. Disengaged: a qualitative study of communication and collaboration between physicians and other professions on general internal medicine wards. BMC Health Serv Res. 2013;13:1-9.

34. Billett S. Learning through health care work: premises, contributions and practices. Med Educ. 2016;50:124-131.

35. Franklin CM, Bernhardt JM, Lopez RP, Long-Middleton ER, Davis S. Interprofessional teamwork and collaboration between community health workers and healthcare teams: An integrative review. Health Serv Res Manag Epidemiol. 2015;2: 1-9.

36. Gruppen LD, Irby DM, Durning SJ, Maggio LA. Conceptualizing learning environments in the health professions. Acad Med. 2019;94:969-974.

37. Reeves S, Xyrichis A, Zwarenstein M. Teamwork, collaboration, coordination, and networking: Why we need to distinguish between different types of interprofessional practice. J Interprof Care. 2018;32:1-3.

38. Liljedahl M, Björck E, Ponzer S, Bolander Laksov K. Navigating without a map: how medical students interact with clinical learning environments. Stud High Educ. 2019;44:275-286.

39. Schrewe B, Ellaway RH, Watling C, Bates J. The contextual curriculum: Learning in the matrix, learning from the matrix. Acad Med. 2018;93:1645-1651.

40. Oandasan I, Reeves S. Key elements for interprofessional education. Part 1: The learner, the educator and the learning context. J Interprof Care. 2005;19:S21-S38.

41. Croker A, Smith T, Fisher K, Littlejohns S. Educators' interprofessional collaborative relationships: helping pharmacy students learn to work with other professions. Pharmacy. 2016;4:17.

42. Dornan $\mathrm{T}$, Tan $\mathrm{N}$, Boshuizen $\mathrm{H}$, et al. How and what do medical students learn in clerkships? Experience based learning (ExBL). Adv Health Sci Educ Theory Pract. 2014;19:721-749. 
Appendix. Example of interprofessional collaborator and communicator role mapping process

STEP 1: Researcher reviews participant role-related diary entries recorded throughout the clerkship

STEP 2a: Researcher reviews the AFPC Framework to locate and consider potential match

STEP 2a: Researcher reviews the CIHC IPE Framework to locate and consider potential match

\section{Potential outcomes:}

$\square$ Present as a concept in the frameworks

$\square$ Absent as a concept in the frameworks
STEP 3: Researcher documents match

STEP 4: Researchers meet to review and debate independent coding to reach final consensus

\section{Diary Excerpt Week 6}

I have had opportunities to develop my skills as a collaborator by attending rounds and by speaking with other healthcare providers when looking at a patient chart. An example of this was being approached by a physician from a consulting service who asked me to conduct a drug information request in order to provide the patient with the best possible safe and effective care.

AFPC Competency Roles: Collaborator AFPC Key and Enabling Competency Descriptions

1. Work effectively with members of the healthcare team including patients, pharmacy colleagues and individuals from other professions.

1.1. Establish and maintain positive relationships

1.2. Recognize, respect, and negotiate the roles and shared/overlapping responsibilities of team members 1.3. Join with others in respectful, effective, shared decision-making

2. Handover the care of a patient to other pharmacy team or nonpharmacy team members to facilitate continuity of safe patient care. 2.1. Determine when and how care should be handed over to another team member

2.2. Recognize, respect, and honour the negotiated shared and overlapping responsibilities of patients, pharmacy team and other health team members when handover occurs

2.3. Demonstrate safe handover of care, using oral, written, and electronic communication, during a patient transition to a different care provider or setting

\section{CIHC Competency Domain: Collaborative Leadership}

To support interprofessional collaborative practice, learners collaboratively determine who will provide group leadership in any given situation by:*

a. work with others to enable effective patient outcomes

b. facilitating effective decision-making

c. collaboratively setting shared plans of care

d. demonstrating respect for all team members, patients, families

Coded as represented in the AFPC competency framework COLLABORATOR: $1(1.1,1.3)$

Coded as represented in the CIHC competency framework COLLABORATIVE LEADERSHIP: $a, b, c, d$

FINAL INTERPROFESSIONAL COLLABORATOR RESULT Coded as represented in the AFPC \& CIHC competency frameworks COLLABORATOR: 1 (1.1, 1.2)

COLLABORATIVE LEADERSHIP: $a, b, d$ 
STEP 1: Researcher reviews participant role-related diary entries recorded throughout the clerkship

STEP 2a: Researcher reviews the AFPC Framework to locate and consider potential match

STEP 2a: Researcher reviews the CIHC IPE Framework to locate and consider potential match

\section{Potential outcomes:}

$\square$ Present as a concept in the frameworks

$\square$ Absent as a concept in the frameworks

STEP 3: Researcher documents match

STEP 4: Researchers meet to review and debate independent coding to reach final consensus

\section{Diary Excerpt Week 6}

When communicating with physicians, I use professional language when presenting evidence-based medicine recommendations. For example, in my recommendation to change to an ICS/LABA I used the GINA guidelines as reference for the physicians.

AFPC Competency Roles: Communicator AFPC Key and Enabling Competency Descriptions

1. Communicate in a responsible and responsive manner that encourages trust and confidence.

1.1. Select appropriate communication strategies

1.2. Provide timely, clear responses tailored to context $\&$ audience

1.3. Express facts, evidence $\&$ opinions accurately \& effectively with clarify \& confidence

1.4. Listen, actively solicit \& respond appropriately to ideas, opinions $\&$ feedback from others

1.5. Use suitable language, pace, tone and non-verbal communication 1.6. Seek \& synthesize relevant information from others in a manner ensuring common understanding

1.7. Compose \& share information optimizing patient safety \& privacy

2. Communicate in a manner that supports a team approach to health promotion and health care.

2.1. Engage ins respectful, empathetic, compassionate, culturally safe, non-judgmental, tactful conversations with others

2.2. Demonstrate awareness of impact of one's own experience, professional culture, biases, on effective working relationships, communication, and conflict resolution with others

2.3. Demonstrate accurate $\&$ appropriate communication and respect for other team member roles when disclosing information about harmful situations

2.4 Convey importance of teamwork in patient-centred care

CIHC Competency Domain: Interprofessional Communication

To support interprofessional collaborative practice, learners are able to:* a. actively listen to other team members

b. communicate to ensure common understanding of care decisions

c. developing trusting relationships with other team members

CIHC Competency Domain: Interprofessional Conflict Resolution

Learners actively engage self and others in dealing effectively with interprofessional conflict

Coded as represented in the AFPC competency framework COMMUNICATOR: $1(1.1,1.3,1.5) ; 2(2.1)$

Coded as represented in the CIHC competency framework INTERPROFESSIONAL COMMUNICATION: b, c,

FINAL INTERPROFESSIONAL COMMUNICATOR RESULT

Coded as represented in the AFPC \& CIHC competency frameworks

COMMUNICATOR: $1(1.1,1.3,1.5) ; 2(2.1)$

INTERPROFESSIONAL COMMUNICATION: b. c

*selected learner competencies in CIHC Domain 



\section{CHAPTER 6}

\section{Discussion}




\section{Discussion}

The aim of this thesis was to enhance understanding of workplace-based assessment and competency development in pharmacy education. The constituent studies address the research questions exploring supervisor, team, and learner expectations of competent clinical work in context. Collectively, they are in effect an examination of curriculum transposition - the movement of curriculum from underpinning ideals $(A)$ and the educational programmes stemming from these (B) through to curriculum implementation in practice (C) and the ultimate effects on learners (D); our studies reveal potentially problematic transformations (between ideal and real) occurring across these three thresholds or planes (Figure 1). ${ }^{1}$ More specifically, the work in the thesis addresses how competency frameworks devised for one health profession (medicine) and adapted by another discipline (pharmacy) are subsequently adopted in a separate geographic context. To do so, we sought to uncover and contrast how key actors in culturally distinct care contexts (clinical supervisors) conceptualize varying levels of student performance and found these workplace-based expectations compatible, but neither faithfully adherent to the outlined competency standards they share. We next illustrated disjuncture that occurs across professional contexts when interpreted competency roles of pharmacist trainees are adjudicated by assessors outside the profession - the multidisciplinary team members working in close proximity to trainees providing care in the clinical learning environment. Finally, we unearthed the narrow representations of developing competencies pharmacist trainees document and reflect upon in the clinical work they conduct on multidisciplinary teams over the course of their hospital workplace-based clerkship.

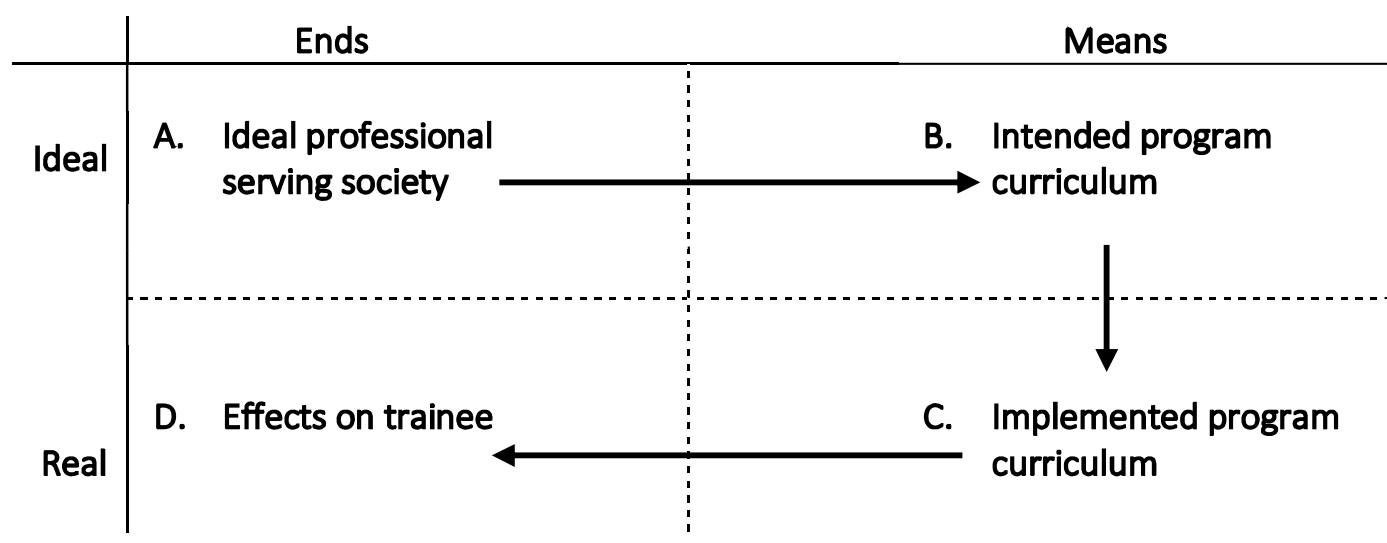

Figure 1. Curriculum transposition framework. Adapted from McCowan. ${ }^{1}$

At the outset, the dissertation was broadly framed around the "people" and "place" influencing competency development and workplace-based assessment in pharmacy (Chapter 1). Bates \& Ellaway, subsequently collaborating with Schrewe et al., consider six contextual patterns and how the intersection of these "people" and "places" inevitably shape trainee learning experiences and outcomes. ${ }^{2,3}$ We return to these specific characterizations of contextual patterns to synthesize and organize the findings and the further questions arising from the thesis studies Figure 2. 


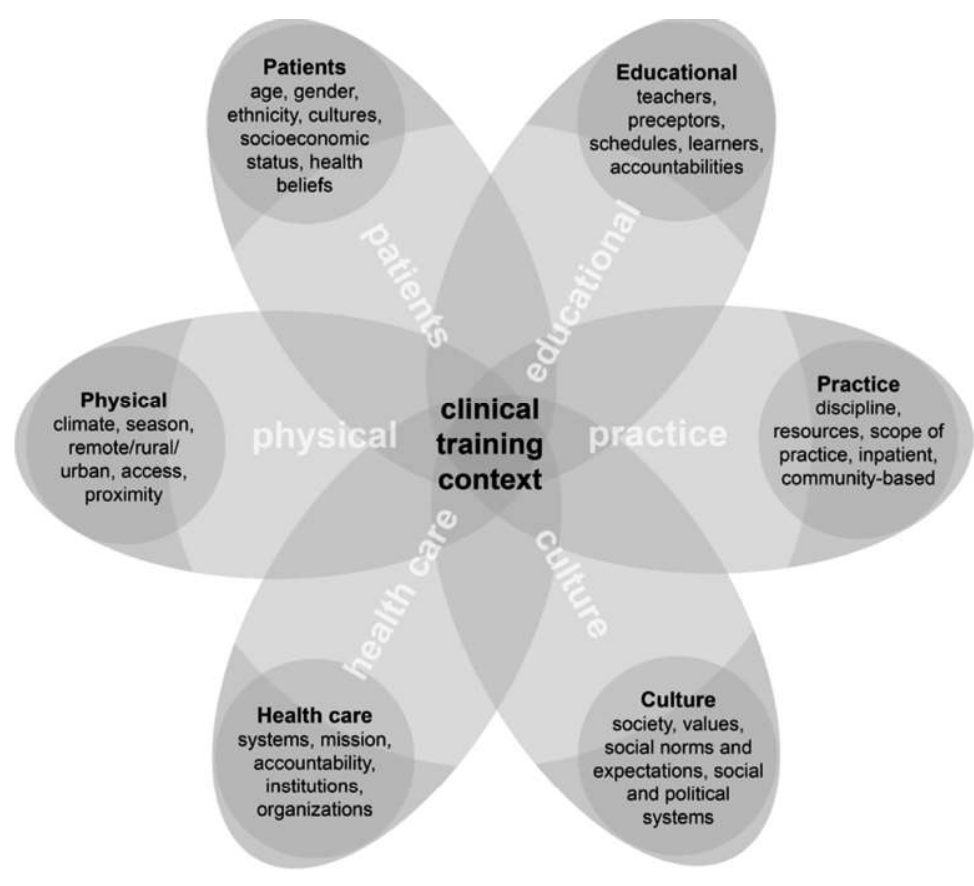

Figure 2. Six contextual patterns of clinical training. Reproduced with publisher permission from Schrewe et al. ${ }^{3}$

\section{Variations in clinical pharmacy supervisors' conceptualization of student performance are not readily attributed to cross-cultural contexts}

\section{People and Place - Culture in Context}

Many before us have explored the impact of diverse geographic contexts on health professional education, but we are among the first to make the foray into the direct patient care spaces and the competency-based assessment of pharmacist trainees. ${ }^{4}$ In a crossborder curriculum, institutions located in different countries strive to offer comparable student learning experiences through transfer of a curriculum (e.g. courses, content, instructional and assessment modalities) across borders for implementation elsewhere. Waterval et al. have laid important groundwork for best principles for establishing and maintaining cross-border health professional education partnerships. Their work cautions that a "shared curriculum" may not be easily integrated into recipients' existing academic structures, including the pedagogical persuasions of its (campus-based) instructors. Meanwhile, Frambach et al. elaborated upon campus-based learner experiences within a transplanted classroom instructional approach (problem-based learning, PBL) concluding cultural aspects of the recipient programs (in the Middle East and Asia) were among the complex contextual factors challenging student learning processes. ${ }^{5}$ Subsequently Wilby et al., examining how communication competency is distinguished in the controlled conditions of an objective structured clinical examination (OSCE) "crossing borders" and administered in a Middle East context, identified assessor cultural orientation as a source of idiosyncrasy in rated pharmacy student performance. ${ }^{6}$ In this thesis, we extend study of such competency 
development and assessment into the actual practice environments of pharmacist trainees. We explore how clinical supervisors conceptualize performance outlined in a Canadianoriented curriculum and thereby investigate its appropriateness for a specific Middle Eastern context.

Our study findings disrupt assumptions that the transplant of a curriculum from its source is inherently susceptible to troublesome recontextualization, as reported for other campusbased aspects of formal learner instruction and evaluation (e.g. PBL, OSCE). Based on these earlier works, we are surprised that the pharmacist trainee competency expectations of clinical supervisors in Qatar and in Canada were not appreciably disparate between-groups given anticipated influences of the relative national societal norms and values predominant in these two broad training contexts as framed in Hofstede's cultural dimensions theory. Indeed, clinical supervisor judgements of described pharmacist trainee performance were similar in how they were dissimilar - meaning that most often the basis of any within-group differences identified were the same ones articulated by participants in both Qatar and in Canada. Discrepant interpretations of competent performance in one country, as set by another nation's standards, were not born out in our results (Chapters 2 and 3). Categorized traits, customs, and belief systems which serve as a backdrop of culture as a core contextual pattern for learner experiences and outcomes (Figure 2) did not emerge as a prevailing factor for variation in expectations of pharmacist trainees. However, we did register departures of enacted competency-based assessment from intended curricular objectives.

\section{People and Place - Education in Context}

We found how clinical supervisors form impressions and judge competent performance in two geographically distinct cultural contexts was largely in agreement, but the dataset contributes to evidence of educational program modifications when implemented in actual clinical learning environments. It is apparent throughout our studies of clinical supervisors, multidisciplinary team members, and pharmacist trainees that formal competency role descriptions are incomplete. Clinical supervisors in Canada and in Qatar form overall student impressions based on performance and behaviours that are not outlined in competency descriptions. Nevertheless, we do not contend this fracture in curricular transposition arises from a mismatch between the competency roles outlined for Canadian medical education and adapted by pharmacy. Like North American clinical supervisors in social work and medicine, pharmacists in our studies also exhibited strong subjective influence of desired student traits (or lack thereof) on competency assessments. ${ }^{7,8}$ "Enthusiasm", "motivation" or "going above and beyond" do not readily blueprint to formally articulated competencies in the educational outcomes of health professionals. Similarly, we do not believe these findings point to a unique unsuitability of the Canadian pharmacy competency-based educational framework for a Middle East clinical training setting. The CanMEDS framework has itself been subjected to conceptual scrutiny and questions of relevance to local daily work when proposed (and subsequently implemented) as a competency-based model for physicians in-training elsewhere in the world. ${ }^{9-12}$ In these instances, we believe dynamic educational contextual patterns are in play regarding the ways programs' stated objectives and assessment criteria (ideal) are implemented across clinical learning environments (real). 
Schrewe et al. suggest the educational contextual pattern encompasses not only how programs are structured (e.g. defined objectives, assessment requirements) and supported (e.g. technologies, roles and attitudes of instructors and co-learners) but how curriculum is enacted (Figure 2). Pharmacist clinical supervisors' conceptualization of pharmacy program competencies, as reflected in their expectations of trainee performance, withstands formal role descriptions. Our studies have therefore seemingly highlighted evidence of another disciplinary (pharmacy) disconnect between the intended and enacted curriculum and associated assessment of students in practice.

\section{Interprofessional team members' service expectations of pharmacist trainees overshadow recognition of discrete collaboration and communication roles}

In this dissertation we do offer novel insight into workplace-based assessment by multidisciplinary team members in the clinical learning environment. Currently, there is relatively little study about competency judgements of trainees by professionals outside their own discipline. In exploring how team members expect pharmacist trainees to demonstrate competent collaboration and communication, we find another disjuncture in curricular transposition between planes (Figure 1) of the ideal and developed educational program and its implementation in practice (Chapter 4). An ethos of shared-care is embodied in the interprofessional competency frameworks that form the basis of curriculum decisions in health professional programs, namely formalized interprofessional education. This philosophy is also denoted in discipline-specific educational outcomes: dietitian, nurse, pharmacist, physician, and physiotherapist trainees must demonstrate abilities to form collaborative relationships with other professionals caring for the same patients. Yet we have identified how expectations and assessment of related pharmacist trainee competencies are reproduced in workplace-based contexts in unforeseen ways.

Unlike clinical supervisory expectations of their discipline-matched trainee that we (pharmacy) and others (physicians, social work) have studied, other health professionals do not appear to judge trainees according to performance that falls outside outlined competency descriptions, per se. For example, multidisciplinary study participants did not use language referencing commitment, disposition, or team-fit to describe the strengths or shortcomings of a pharmacist trainee. On the surface, declared expectations for collaboration and communication are satisfyingly uniform, validating the "interprofessional" status of these competencies. Multidisciplinary team members expect all traineesirrespective of profession - to convey relevant information concisely, make recommendations with confidence, address them with respect. Still, beyond the semantic analysis of interprofessional expectations of pharmacist trainees, we find influencing practice and healthcare contextual patterns. In their descriptions of interactions with trainees outside their own discipline, we actually found a predilection of function over form. Participants generally seemed to judge a pharmacist trainee according to their instrumental value with minimal real attention to the specific collaborator or communicator competencies demonstrated. In various ways, a utilitarian orientation towards shared-care is reflected in our data of pharmacist trainee expectations of competency performance in team settings (Chapter 5). Formal campus-based interprofessional education activities preceding the 
experiential training component of pharmacy programs are purposefully designed to promote interprofessional patient care through early exposure to one another's roles and immersion into simulated exercises of shared-care. We perceive in our data another disjuncture in curriculum transposition, this time at the plane where a program implemented in context affects student abilities and identities in ways not originally envisaged. Specifically, how pharmacist trainees act and are assessed in teams in the clinical learning environment.

\section{People and Place - Practice and Health Care in Context}

Competency-development and assessment in the workplace may be complicated by the relationship between learning and service. Subtle signals of service expectations are embedded in our data from Chapter 2 and 3. When clinical supervisors first identified performance expectations, the categories they conceptualized included pharmacy student organization (e.g. handling and prioritizing multiple tasks), independence (e.g. proactively approaching work or exhibiting extra efforts in assigned tasks), as well as the integration and contributions to team interactions (Chapter 2). These ideals were upheld in Chapter 3 in a different sample of clinical supervisors in Qatar and in Canada who expected pharmacist trainees to be organized, able to manage multiple tasks, and provide relevant information when other team members (usually physicians) were making care decisions related to drug therapy. We considered if our inability to demonstrate differences in pharmacist trainee competency expectations among clinical supervisors in Qatar and in Canada was in part because they were obscured by a unifying professional culture transcending these geographically distinct inpatient environments. We pivoted then to explore pharmacist competency expectations from the perspective of different multidisciplinary team members working in proximity with pharmacist trainees in the clinical learning environment. In these interprofessional responses, the glimpse of intraprofessional service expectations in our first studies becomes a glare (Chapter 4 ). While they profess to expect pharmacist trainees to demonstrate the same fundamental collaborator and communicators competencies as any other learner on their unit, the responses belie a different reality. The episodes of daily care pharmacist trainees chose as representative samples of communication and collaboration in Chapter 5 also portray their working relationships with multidisciplinary team members as transactional. If team-based competencies were a love language, pharmacist trainees would express theirs through the provision of information to physicians! ${ }^{13}$

\section{Pharmacist trainees experience narrow scopes of interprofessional collaboration and communication in the inpatient workplace-based settings}

We intentionally avoid labelling the relationship between service and learning as a tension. ${ }^{14,15}$ Without a doubt, health professionals and trainees must often navigate the competing demands of patient care in busy settings doubling as clinical learning environments. Clinical supervisors feel taxed for time to teach, directly observe care, and engage in feedback conversations with trainees. ${ }^{16}$ Together with the disposition of said health professionals, these circumstances are elemental to Schrewe et al.'s practice 
contextual patterns. They additionally intersect with health care contextual patterns whereby organizational values, rules, and routines impact learning. We ascribe to the notion that trainees learn through work which is synonymous with service. ${ }^{17,18}$ Consistent with the overarching social constructivist and social cultural perspectives throughout this thesis, we consider even service-oriented interactions with members in the environment as a means to create knowledge. Clinical supervisors in the hospital team settings we studied illustrate this approach, as recorded in pharmacist trainee clerkship diaries in Chapter 5. Trainees reported how they discussed their patient assessments, understanding of drug therapy, and interpretations of published evidence with their supervisors who further prepared them for the subsequent interprofessional communication and collaboration. These team encounters were often followed by a debrief between pharmacist clinical supervisor and trainee. However, service-learning perspectives of team member and students themselves are less clear and may point to elements of ambiguity surrounding pharmacist trainee roles on teams. These coached interprofessional encounters were almost entirely oriented to pharmacist trainee interactions with physicians. Pharmacist trainee engagement with other team members in the clinical learning environment is considerable in information exchange, but appears deficient in authentic shared-care. Consequently, performance expectations of pharmacist trainees and associated competency judgements are latently service-oriented (Chapters 4). As we write in Chapter 5, broader orbits of cooperation among team members for patient care exist separate from physicians, but interprofessional communication and collaboration by the pharmacist trainee gravitates towards the prescriber. Understanding how pharmacist trainees negotiate these liminal spaces and its impact on intra- and interprofessional competency development is necessary to optimize symmetry between service and learning on inpatient teams.

\section{Implications}

Findings in this thesis have broadened our understanding of pharmacy competency development and workplace-based assessment with future implications for how: culture is invoked to study and compare training contexts; teams can contribute to multisource feedback; and competency expectations may be used to characterize and recalibrate a curriculum.

The dissidence between consistent workplace-based competency expectations found in our studies and culturally-attributed idiosyncrasies reported during structured competency assessment (OSCE) in other research- work both situated at the same Canadian-accredited Middle East pharmacy school - is notable. ${ }^{6}$ We have argued in this thesis how the intersection of "people" and "place" shape perspectives on pharmacist trainee competency development in the clinical learning environment. Practically speaking, perpetually shifting relationships across the six contextual patterns thwarts consistency in specific opportunities for trainee development and demonstration of competencies. ${ }^{19}$ Yet, this very goal is inherent to the objectives of program partnerships and shared curriculum across borders. From a standardization perspective then, convergence of trainee performance expectations between practice-based assessors in disparate geographic contexts (as seen in our studies) would assuage concerns that local curricular adaptations may render it paradoxically 
misaligned from its source. Achieving the right balance has favorable broader consequences for institutions and individuals (e.g. program accreditation, recognition and transfer of trainee credentials). Despite reassuring prospects arising from these results, we discourage ongoing exploration with positivist-oriented tools. More nuanced approaches are necessary to appreciate how clinical supervisor cultural identities or predispositions acting as a potential independent variable in workplace-based assessment can be responsibly categorized. Processes through which researchers contextualize a phenomenon are tied to the distinctions made across boundaries. Sensemaking of context through a fixed analytical construct (e.g. cultural dimensions framework) is likely incomplete. This assertion is recognizable in our 'within country' clinical supervisor data: competency constructs and associated performance expectations by individuals within a national context, or even the same clinical learning environment, is not a monolith. Our lessons from comparing culture in context and findings of disruptions in curricular transposition also have implications for programs which do not partner across-borders, but promote "internationalization" of their curriculum. Although purposeful integration of intercultural dimensions into health professional education to enhance quality and preparation of the "global" graduate is commendable, we caution against assumptions and resultant miscasting of which knowledge, skills, and attitudes are privileged (by and for whom). ${ }^{20,21}$

Assumptions about the universality of interprofessional competency roles were also challenged in this dissertation. We questioned whether standard communication skills and collaborative behaviours incorporated in the classroom-based interprofessional education of all health trainees might actually be judged differently by practicing multidisciplinary team members. Discrepant interpretations of shared care competencies outlined in interprofessional frameworks would have implications for multisource feedback in the clinical learning environment. Instead, our inability to delineate meaningful differences in multidisciplinary team member constructs for collaboration and communication demonstrated by trainees outside their own profession were obscured by service-related expectations. These findings suggest workplace-based assessment of pharmacist and other health professional trainees joining teams may be undermined in unexpected ways. Team members' competency judgements bound principally to efficient and relevant information sharing illustrates potential disagreement between an idealized concept of shared-care and/or how multidisciplinary trainees in a clinical learning environment contribute. Such perspectives may limit trainee interprofessional communication and collaboration experiences or cultivate pre-existing narrow views. When combined with infrequent routine encounters between non-physician trainees and non-physician team members, the capacity for substantive feedback of interprofessional competency development and performance (formative or otherwise) is lacking. Broad, retrospective, informal solicitation of multisource feedback at best yields a global impression of the multidisciplinary trainee and often, no impression at all. We propose then for authentic and specific performance-relevant information to reach trainees, distinct episodes of patient care be coordinated with team members outside their discipline. For example, Min et al. have previously written about how emergency medicine residents leading patient/family meetings received structured formative assessment from the social worker present immediately afterwards. ${ }^{22}$ This type of deliberate practice with interprofessional team members should be considered for 
integration into other busy clinical learning environments to judge and foster trainees' developing communication or collaborative tasks.

Throughout the dissertation, the interpretations and impressions of pharmacist trainee competencies were examined through participant expectations. ${ }^{23}$ Social scientists characterize how observers form inferences about human behaviours using different approaches and these may be linked to satisfaction. For example, according to certain business and marketing theory, individuals possess initial expectations of a specific product or service and form perceptions about its performance after a period of consumption or experience. When actual performance confirms positive expectations, individuals are satisfied. Conversely, dissatisfaction is the resultant negative affect of unmet or controverted expectations. ${ }^{24}$ As a variation on this premise, the data collection and analysis in our studies were oriented to explore what discrepancies might exist between a participant's standard of trainee performance (expectations) and perceived outcome (met or unmet expectations) and how this may influence workplace-based assessment (expressed satisfaction). ${ }^{25}$ Few researchers have previously taken a social constructivist view to understand processes supervisors in the health professions use to assess trainee performance, as marked by their stated performance expectations. ${ }^{26}$ Expectations as a construct are embedded in many psychological theories, but appear frequently as a function in mathematical models to be predicted or estimated in relation to satisfaction. ${ }^{23}$ Together, these mixed-model approaches could be useful tools in the evaluation of curricular transposition - the materialization of educational program ideals into outcomes. Reasons construct alignment between competency assessment and competency standards deviate in practice are plentiful and complex; findings from these thesis studies further add to this body of work. Continuing to frame competency-based health professional education research as questions of stakeholder (faculty, supervisors, students, patients, and employers) expectations is not only a useful approach to understand discrepancies between the ideal and real health professional program curriculum, but to stimulate re-examination of its principles; the illustrated trajectory of curriculum disposition across planes (Figure 1) should not in reality be fixed in one direction.

\section{Strengths and Limitations}

The strengths and limitations of the individual studies constituting this thesis are addressed in their respective chapters. What follows are supplementary considerations and conscientious reiteration of issues pertinent to the program of research as a collective.

The dissertation work spans two comparator geographic regions that must be acknowledged to have internal variance. Specifically, the qualitative nature of the data mostly collected from one country (Qatar) restricts direct extrapolation of findings to workplace learning settings in other Middle Eastern countries where cross-border curriculum partnerships have been established (Chapters 2 and 3). Although we sampled widely across Canada as our contrasting representative "Western" nation (Chapter 3), we recognize that that this too is certainly not a uniform view. Similarly, the studies conducted within pharmacy training from one large program (Chapters 4 and 5) and within one particular team-based setting (Chapter 
4) warrants any claims that findings might hold for other health professional education or service populations to be made judiciously. ${ }^{27}$

The research questions forming this dissertation were explored through complementary socio-cultural and social-constructivist theoretical perspectives, research paradigms that lend themselves to naturalistic data collection. Consequently, ethnographic study would have been suitable to explore these workplace-based research questions - all issues embedded within multiple systems or sectors. ${ }^{28}$ Ethnography as a framework for qualitative inquiry is particularly useful to inform the interpretation of the culture of the group, community or setting. In these studies, we often asked participants to describe their perspectives, but as researchers, we understand that explanation of beliefs may not be consistent with actual behaviours that might be directly observed. Unfortunately, resources to conduct fieldwork appropriately and the time to identify and address ethical considerations were not available. We did avail ourselves to a host of other qualitative and quantitative methods. This omnivorous approach to data collection strategies - content mapping (Chapters 2 and 5), interviews (Chapters 2 through 5), Delphi nominal and other consensus processes (Chapters 3 and 5), and written diaries (Chapter 5) - represented an integrative approach to examining the phenomenon of interest through more than one lens. ${ }^{29}$ Used in concert, these approaches to the dissertation topic permitted use of results to elaborate, inform, and corroborate the subsequent data collection method. ${ }^{30}$ The multimethod program of work was also unified by a constructivist stance.

This dissertation integrates studies exploring the answer to an overarching question: how can we understand performance expectations to enhance competency development and workplace-based assessment of health professional trainees? Our findings simultaneously draw pharmacy into and advance ongoing critical conversations about experiential training which intersects with broader health professional education scholarship. A strength of this research is its practical relevance to adjacent topics concerning authentic work-integrated learning in diverse clinical environments and performance feedback. In Chapters 2 and 3, we studied previously unexplored aspects of cross-border health professional curriculum partnerships - outside the classroom and at inpatient training practice settings. In Chapters 4 and 5 , our study of communication and collaboration competencies uncovered utilitarian relationships between learners and multidisciplinary team members. All studies are underpinned by issues salient to pharmacist trainee roles and interprofessional care.

Indeed, the healthcare settings where this research takes place are not only rich contexts for collaborative teamwork, but also environments for interaction and engagement around health professional education and research questions. It is only fitting then that a diverse research team has undertaken this work to broaden understanding of workplace-based assessment and competency development in pharmacy education. My pharmacy and public health qualification was bolstered by the supervision of an experienced leadership team with diverse backgrounds and training in medical clinical practice, education and their associated program and policy development. Although European-based, the senior researchers have previously explored medical education research questions in Middle East countries. The dissertation (and principal investigator reflexive distance) was also supported by research personnel who participated in data collection and analysis with strong understanding of their 
own local contexts (Chapters 2,4 and 5) and proximity to the health professional trainee perspective (Chapters 2 and 5). In Chapter 4, early coding and analysis was undertaken with an international (native of Asia Pacific country) graduate student in the department of education who possessed a business management background. This network of diverse disciplinary knowledge, skills, and relationships to the data augments the resultant evidence base.

In contrast to earlier stated caution about generalizing the findings of this thesis work, its transferability is certainly germane. Unlike generalizability, transferability does not involve broad claims, but invites readers of research to make connections between elements of a study and their own experience. ${ }^{31} \quad$ Although it is the responsibility of the outside researcher/reader to determine transferability to their own setting and circumstances, those judgements can be facilitated by the source researcher/writer. The dissertation accomplishes this by providing thick descriptions, full purposeful accounts of the contexts (cultural, social, organizational) of data collection across the four studies and using purposive sampling, selecting (and describing) relevant professional and student participants to inform the communities under study.

As intimated in the reflexivity testimony of Chapter 1, this work undoubtedly profited from other interprofessional and education-oriented collaborations in which I was engaged throughout the doctoral study. The disciplinary scope of these scholarly partnerships further broadened upon return to Canadian academia in these latter years. As a University of British Columbia Green College Leading Scholar, I joined a program that fostered dialogue with other newly appointed faculty members and the appreciation of research across botany, computer science, engineering, French literature, German media study, public policy and global affairs, social justice, visual art and history, just in my cohort alone. ${ }^{32}$ The latter parts of this thesis drew some surprising parallels with the examination of $12^{\text {th }}$ century romance poems and the study of plankton communities!

\section{Future Research}

Researchers looking to seize the next opportunities to study contextual influences on competency development and assessment and the repercussions on intended health professional curriculum are spoiled for choice. Obvious and specific directions springing from these thesis studies include further examination of workplace-based training from the international vantage point. Globalized health professional education continues to proliferate and critical analysis of cross-border partnerships remains relevant. Studies attending to competency development and assessment in practice should be pursued in other health disciplines whose professional curriculum (and associated principles, processes, content, documents, etc.) are shared and implemented across different national jurisdictions. Such program models are additionally increasingly mobilized to other countries from non-Western (e.g. Australian, British, European, North American) origins. ${ }^{33}$ We recommend sequel projects expand upon our research questions to explore the reciprocal influences that trainees emerging from cross-border partnership programs have on the local clinical learning and patient care environments. 
The science of teamwork is steeped with study of how health care teams function to deliver safe and effective patient care. ${ }^{34}$ The evidence demonstrates that performance is generally predicted by how members cooperate and not individual expertise. ${ }^{35}$ Our data exploring pharmacist trainee workplace-based competency expectations tendered provocative insights into pharmacist roles on inpatient teams. In many respects, pharmacists did associate their team role with medication knowledge and information - as reflected in clinical supervisory expectations and in trainee clerkship diaries of clinical work. How this expertise was shared outside formal team structures (e.g. discharge rounds, bedside rounds) and by which health professionals it was routinely required for decision-making appeared quite narrow. Pharmacist trainee practice expectations of interprofessional communication and collaboration skewed heavily towards physicians. Orbits of team cooperation exist whereby nurses and other multidisciplinary members engage in rich, patient-focused problem-solving and care planning, separate from physicians. ${ }^{36}$ It is presently unclear what influences where pharmacists (and by extension, their trainees) are situated and what significance this actual or perceived positioning has on working relationships and the enacted shared-care roles envisioned in pharmacy and interprofessional competency frameworks.

The patient as a pertinent stakeholder possessing expectations of pharmacist trainee competency in the workplace was absent from the dissertation's explicit research questions. With wide-ranging underlying conditions, symptoms and illness presentations, prior and future treatment courses, socio-demographic make-up, ethnic backgrounds and many other individual traits, patients collectively form one of the six key contextual pattern in the clinical learning environment. ${ }^{2,3}$ In a health professional program, patients are in essence the alpha and omega - ideal curriculum are conceived to meet societal needs and subsequently designed to train graduates who deliver the best care according to existing and emerging requirements of communities and populations. ${ }^{37}$ Patients are therefore not simply in the contextual foreground shaping trainee competency development, they are the ultimate "end users" of this training. Understanding what demonstrated competencies patients and families prioritize during care encounters and expanding on how these perspectives are incorporated into trainee workplace-based assessment is necessary in the ongoing study of curriculum transposition in health professional education. 


\section{Chapter 6}

\section{Conclusions}

Clinical learning environments are dynamic sites where health professionals, support personnel, and trainees assume shifting and time-sensitive daily tasks to care for the acute and chronic health issues of diverse patient populations. It is in these workplace-based settings where the intended professional program competencies taught in classrooms and represented in simulations are enacted in actual practice - "no where now here". Using a model of six patterns of contextualization to synthesize and organize our findings of workplace-based assessment and competency development in pharmacist training, we have characterized expectations for pharmacy trainees, especially their roles on hospital healthcare teams. Performance judgements stray from the competency role descriptions identified across broad inpatient contexts arising from clinical supervisors (intradisciplinary), team members (multidisciplinary), and the trainees themselves. Reconciliation of the prescribed pharmacy and interprofessional curriculum and the competency development and assessment occurring in workplace-based training entails understanding how "people" and "place" shape these transformations with reciprocal and pragmatic review of program aims and articulation of the competencies themselves. 


\section{References}

1. McCowan T. Curricular transposition in citizenship education. Theory Res Educ. 2008;6:153-172.

2. Bates J, Ellaway RH. Mapping the dark matter of context: a conceptual scoping review. Med Educ. 2016;50:807-816.

3. Schrewe B, Ellaway RH, Watling C, Bates J. The contextual curriculum: Learning in the matrix, learning from the matrix. Acad Med. 2018;93:1645-1651.

4. Waterval DG, Driessen EW, Scherpbier AJ, Frambach JM. Twelve tips for crossborder curriculum partnerships in medical education. Med Teach. 2018;40:514-519.

5. Frambach JM, Driessen EW, Chan LC, van der Vleuten CPM. Rethinking the globalisation of problem-based learning: how culture challenges self-directed learning. Med Educ. 2012;46:738-747.

6. Wilby KJ, Govaerts MJ, Austin Z, Dolmans DH. Exploring the influence of cultural orientations on assessment of communication behaviours during patient-practitioner interactions. BMC Med Educ. 2017;17:61.

7. Bogo M, Regehr C, Woodford M, Hughes J, Power R, B RG. Beyond competencies: Field instructors' descriptions of student performance. J Soc Work Educ. 2006;42:579-593.

8. Ginsburg S, Mcllroy J, Oulanova O, Eva K, Regehr G. Toward authentic clinical evaluation: pitfalls in the pursuit of competency. Acad Med. 2010;85:780-786.

9. Ringsted C, Hansen TL, Davis D, Scherpbier A. Are some of the challenging aspects of the CanMEDS roles valid outside Canada? Med Educ. 2006;40:807-815.

10. Sottas B. Learning outcomes for health professions: the concept of the swiss competencies framework. GMS Z Med Ausbil. 2011;28:11.

11. van der Lee $\mathrm{N}$, Fokkema JP, Westerman $\mathrm{M}$, et al. The CanMEDS framework: relevant but not quite the whole story. Med Teach. 2013;35:949-955.

12. Jilg S, Möltner A, Berberat P, Fischer MR, Breckwoldt J. How do supervising clinicians of a university hospital and associated teaching hospitals rate the relevance of the key competencies within the CanMEDS roles framework in respect to teaching in clinical clerkships? GMS Z Med Ausbil. 2015;32:33.

13. Chapman GD. Chapman GD. The five love languages: The secret to love that lasts. Farmington Hills, MI: Walker Large Print; 2010

14. Cleland J, Durning SJ. Education and service: how theories can help in understanding tensions. Med Educ. 2019;53:42-55.

15. Sholl S, Ajjawi R, Allbutt $H$, et al. Balancing health care education and patient care in the UK workplace: a realist synthesis. Med Educ. 2017;51:787-801. 
16. Watling C, LaDonna KA, Lingard L, Voyer S, Hatala R. 'Sometimes the work just needs to be done': socio-cultural influences on direct observation in medical training. Med Educ. 2016;50:1054-1064.

17. Sholl S. Balance or tension? Walking the tightrope between training and patient care. Med Educ. 2018;52:248-249.

18. Teodorczuk A, Ajjawi R, Billett S, Hilder J, Noble C. The service/teaching tension: a window into the soul of a hospital. Med Educ.2018;52:678-678.

19. Bates J, Schrewe B, Ellaway RH, Teunissen PW, Watling C. Embracing standardisation and contextualisation in medical education. Med Educ. 2019;53:15-24.

20. Hanrieder T. How do professions globalize? Lessons from the global south in US medical education. Int Political Sociol. 2019;13:296-314.

21. Prideaux D. The global-local tension in medical education: turning 'think global, act local' on its head? Med Educ. 2019;53:25-31.

22. Min AA, Spear-Ellinwood K, Berman M, Nisson P, Rhodes SM. Social worker assessment of bad news delivery by emergency medicine residents: a novel direct-observation milestone assessment. Intern Emerg Med. 2016;11:843-852.

23. Maddux JE. Expectancies and the social-cognitive perspective: Basic principles, processes, and variables. In: How expectancies shape experience.: American Psychological Association; 1999:17-39.

24. Jiang JJ, Klein G. Expectation-confirmation theory: Capitalizing on descriptive power. In: Handbook of research on contemporary theoretical models in information systems. IGI Global; 2009:384-401.

25. Hogan EA. Effects of prior expectations on performance ratings: A longitudinal study. Acad Manage J. 1987;30:354-368.

26. St-Onge $C$, Chamberland $M$, Lévesque $A$, Varpio L. Expectations, observations, and the cognitive processes that bind them: expert assessment of examinee performance. Adv Health Sci Educ. 2016;21:627-642.

27. Carminati L. Generalizability in qualitative research: a tale of two traditions. Qual Health Res. 2018;28:2094-2101.

28. Reeves S, Peller J, Goldman J, Kitto S. Ethnography in qualitative educational research: AMEE Guide No. 80. Med Teach. 2013;35:1365-1379.

29. Multimethod Research. In: Lewis-Beck M, Bryman A, Futing Liao T, eds. The SAGE Encyclopedia of Social Science Research Methods. Thousand Oaks, CA: Sage Publications, Inc.; 2004.

30. Bazeley P. Integrative analysis strategies for mixed data sources. Am Behav Sci. 2012;56:814-828.

31. Jensen D. Transferability. In: Given LM, ed. The SAGE Encyclopedia of Qualitative Research Methods. Thousand Oaks, CA: SAGE Publications, Inc.; 2012. 
32. Green College Leading Scholars Program. https://greencollege.ubc.ca/green-collegeleading-scholars-program Web site. Published 2020. Accessed 27 May, 2020.

33. Zhang L, Kinser K, Shi Y. World economies and the distribution of international branch campuses. Int High Educ. 2014:8-9.

34. Rosen MA, DiazGranados D, Dietz AS, et al. Teamwork in healthcare: Key discoveries enabling safer, high-quality care. Am Psychol. 2018;73:433.

35. Salas E, Reyes D, McDaniel S. The science of teamwork: Progress, reflections, and the road ahead. Am Psychol. 2018;73:593-600.

36. Zwarenstein M, Rice K, Gotlib-Conn L, Kenaszchuk C, Reeves S. Disengaged: a qualitative study of communication and collaboration between physicians and other professions on general internal medicine wards. BMC Health Serv Res. 2013;13:1-9.

37. Gruppen L, Mangrulkar R, Kolars J. Competency-based education in the health professions: Implications for improving global health. Hum Resourc for Health. 2012;10:43. 



\section{Summary}

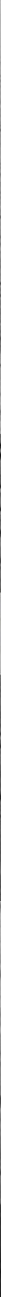




\section{Summary}

The essential role of workplace-based training in health professional competency development is incontrovertible. Conducted in authentic, yet varied patient care settings, trainees have the opportunity to reinforce and shape development of existing knowledge and skills under the supervision of clinical mentors. During hospital clerkships, pharmacy students join multidisciplinary teams to collaborate with other professionals and provide patient care. The dynamic contextual patterns - which feature culture, health care, practice, patients, educational, physical - make workplace-based settings rich clinical learning environments. The "people" and "place" ultimately impacts what trainees do and how performance is judged. Consequently, pharmacist clinical supervisors and adjacent health professionals possess performance expectations informing workplace-based assessment in ways that may overshadow the competencies described in discipline-specific or interprofessional educational frameworks. Such departures from the intended program curriculum influences trainees in complex ways. This thesis sought to gain a broader understanding of workplace-based assessment and competency development in pharmacy education.

Chapter 2 sought to explore how clinical preceptors conceptualise varying levels of pharmacy student performance. Using constructivist grounded theory, semi-structured interviews were conducted with supervisors contributing to workplace-based training of students in North America and supervisors who train students enrolled in these programs delivered in the Middle East (cross-border curriculum). We found seven major themes representing expectations of student performance - knowledge, team interaction, motivation, skills, patient care, communication, professionalism - and these assessment criteria were congruent across these different cultural contexts. Understanding how perceptions of student performance may vary among preceptors recruited in different countries is an essential part of quality assurance for programmes using transplanted or sharing a curriculum. However, just like prior study of physicians and social workers, pharmacists formed overall student impressions based on performance and behaviours that were both holistic and not necessarily outlined in intended educational outcomes. These results highlight the ongoing challenges to integrate relevant practice-based value judgements in the assessment of health professional competencies are also faced by pharmacy internationally.

Chapter $\mathbf{3}$ delves further into the examination of these workplace-based performance expectations of pharmacy trainees by supervisors in hospital practice. Student composites or "vignettes" were developed from the authentic supervisory experiences shared in participant interviews from our study reported in Chapter 2. We employed a mixed-methods design using Delphi consensus measurement to first elicit supervisor characterizations of the performance described in the vignettes followed by interviews to gain greater insight into assessor rationale for these judgements. Hofstede's cultural dimensions theory served as the framework for comparison of responses by the clinical preceptors across two distinct contexts (Canada and Qatar) who participated. Discrepant assessor judgements anticipated by these countries' contrasting predominant societal norms and values were not borne out in our analysis. While within-country group disagreement for certain descriptions of student 
performance was found, the consensus threshold for between-country group differences in supervisory impressions was met for only two of the sixteen student vignettes. We did not find that Middle East culture appreciably informed Qatar clinical preceptor characterization of trainee performance. Supervisors encouraged independence in patient care, discounted some elements of team harmony, and expected trainees to challenge physician authority when appropriate. The cultures influencing assessor judgements were instead more likely to be associated with the professional practices and organizational environments in which these students and supervisors were situated.

In light of a plausible shared professional culture influencing performance expectations demonstrated across geographic settings, Chapter $\mathbf{4}$ shifts to understanding how pharmacy trainees are judged from different disciplinary perspectives. Competencies related to communication and collaboration necessary for inpatient care are largely considered interprofessional in nature and hence trainees' performance considered suitable for team feedback. While enlisting different viewpoints could indeed yield valuable insights for workplace-based assessment, doing so without appreciating the frames of reference from which they arise could be problematic. Using a constructivist grounded theory approach, we conducted semi-structured interviews with fourteen multidisciplinary team members of the acute medicine units at a major teaching hospital in Canada. The study identified that team members conceptualized communication and collaboration of pharmacy and other trainees outside their own profession according to described standards or best practices. Yet in the examples they invoked, team members seemingly judged a trainee's expected performance for its instrumental utility and not by the specific communicator and collaborator competencies the individual demonstrated. Professed sentiment of support for trainees' interprofessional competency development on their clinical teaching unit was admittedly undermined by the episodic nature of encounters with learners outside their own discipline. Infrequent interactions and service-oriented expectations of trainees by inpatient team members reveal potential shortcomings of multidisciplinary contributions to workplacebased assessment.

By Chapter 5, we reach the perspective of the pharmacy trainees themselves and the performance expectations in workplace-based settings. In this study we positioned the trainee at the center for the meaning-making experience within the social context of teambased patient care. During the hospital clerkship of their graduating year, pharmacy trainees maintained written diaries documenting interprofessional communication and collaboration experiences. These records, and the transcripts of follow-up interviews, were analyzed using reflexive thematic analysis. While reported clerkship activities represented most collaborator and communicator roles outlined in pharmacy and interprofessional competency frameworks, routine interaction with other health professionals was largely absent; team encounters were predominantly with physicians. Pharmacy trainees infrequently engaged in authentic interdependent care, instead usually seeking patient data or offering medication information to others. These interactions were almost completely devoid of perceived interpersonal or role conflict. Our results offer insight into how pharmacist trainees perceive and develop competencies for team-based care. Further work is required to understand how such limited scope of interprofessional communication and collaboration might ultimately impair quality patient care. 
In Chapter 6, we synthesize the main findings from all chapters and organize how the research questions have been addressed using the six contextual patterns evident in clinical learning environments. First, varied conceptualization of student workplace-based performance was not identified among clinical supervisors training pharmacy students according to a shared competency framework, yet in distinct national cultural contexts. When next exploring expectations of competent trainee collaboration and communication across professional contexts, multidisciplinary team members exposed latent serviceoriented outlooks. Finally, the predominant interprofessional collaboration and communication roles featured in pharmacist trainee recorded practice experiences belie a predisposition for drug information transmission to physicians. The broader implications of supervisor, team, and trainee expectations for demonstrated competencies in the workplace and consequences for assessment and outcomes of the intended pharmacy curriculum are considered. These include how: culture is invoked to study and compare training contexts; teams can contribute to multisource feedback; and competency expectations may be used to characterize and recalibrate a curriculum. We submit the strengths and limitations of the work presented in the dissertation and propose compelling avenues for future research. 



\section{Samenvatting}

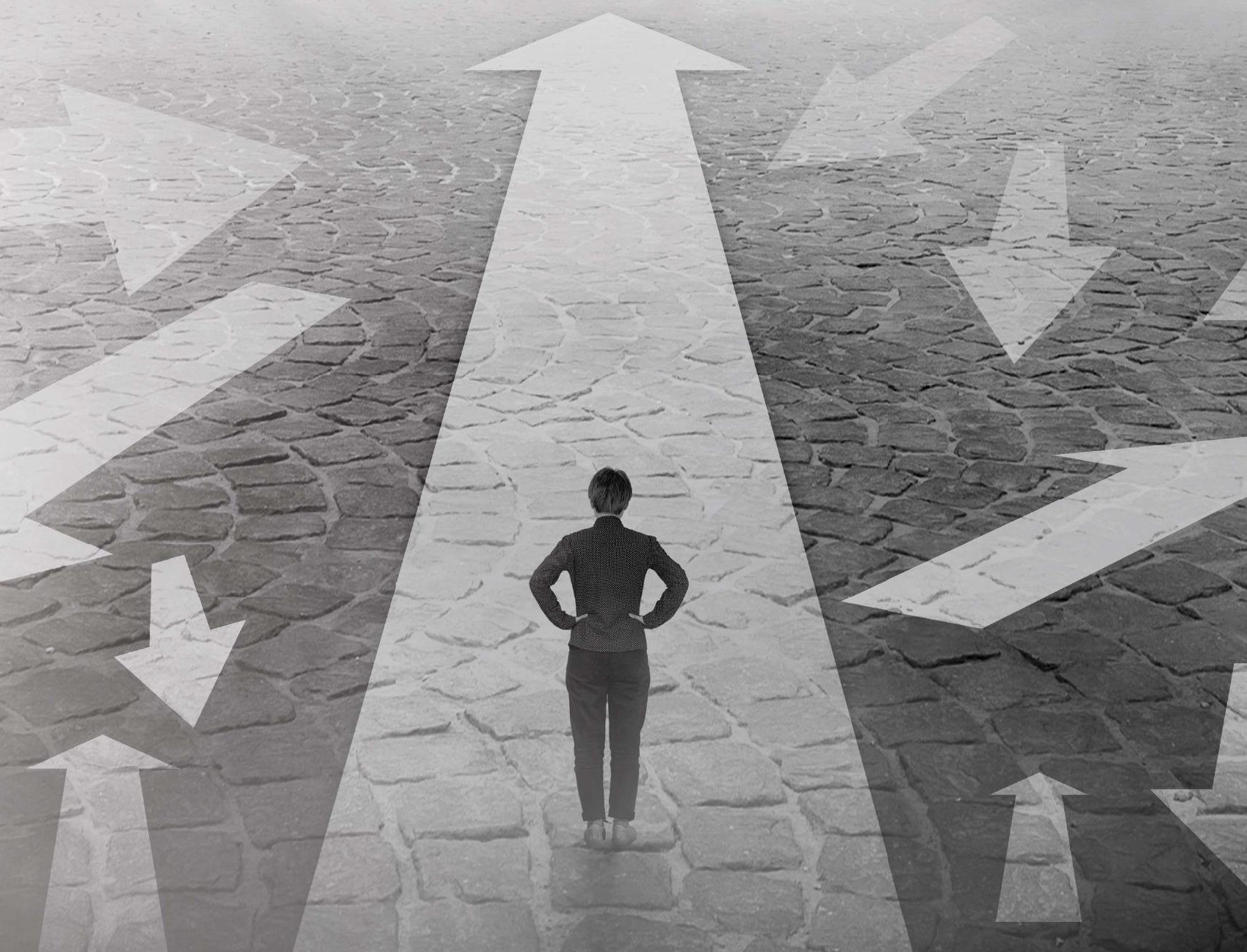




\section{Samenvatting}

Dat opleiden op de werkplek een essentiële rol speelt in de ontwikkeling van competenties bij zorgprofessionals staat buiten kijf. Doordat het opleiden plaatsvindt in een authentieke doch gevarieerde patiëntenzorgsetting krijgen coassistenten de kans om onder begeleiding van klinische mentoren de ontwikkeling van bestaande kennis en vaardigheden te versterken en vorm te geven. Tijdens coschappen in het ziekenhuis maken studenten Farmacie deel uit van multidisciplinaire teams waarbinnen zij met andere professionals samenwerken teneinde patiëntenzorg te verlenen. De dynamische omgevingspatronen, waaronder cultuur, de zorg, praktijk, patiënten, onderwijs en de fysieke omstandigheden, maken van de werkplek een rijke klinische leeromgeving. Het zijn uiteindelijk de "mensen" en de "plek" zelf die beïnvloeden wat de coassistenten doen en hoe hun functioneren wordt beoordeeld. Klinische apotheker-supervisoren en aanpalende zorgprofessionals hebben dan ook prestatieverwachtingen die de werkplekbeoordeling dusdanig beïnvloeden dat zij de competenties die in de discipline-specifieke of interprofessionele onderwijskaders zijn beschreven, kunnen verbloemen. Dergelijke afwijkingen van het beoogde opleidingscurriculum hebben een complexe uitwerking op de coassistent. Het doel van dit proefschrift was om een ruimer begrip te krijgen van werkplekbeoordelingen en competentieontwikkeling in het farmacieonderwijs.

In Hoofdstuk 2 werd onderzocht hoe klinisch docenten de diverse niveaus van presteren door studenten Farmacie conceptualiseren. Met behulp van constructivistische, gefundeerde theorie werden er semigestructureerde interviews gehouden met supervisoren die hadden meegewerkt aan het opleiden van studenten op de werkplek in Noord-Amerika en met supervisoren die studenten opleidden die voor deze programma's die in het Midden-Oosten werden aangeboden, ingeschreven stonden (grensoverschrijdend curriculum). We stelden zeven centrale thema's vast die hun verwachtingen van de prestaties van studenten vertegenwoordigden: kennis, teaminteractie, motivatie, vaardigheden, patiëntenzorg, communicatie en professionaliteit. De beoordelingscriteria die in deze verschillende culturele contexten werden gehanteerd, waren bovendien in overeenstemming met elkaar. Een essentieel onderdeel van de kwaliteitszorg voor opleidingen die gebruik maken van een geëxporteerd curriculum of die een curriculum delen, is begrijpen hoe percepties van studentprestaties kunnen verschillen onder docenten die in verschillende landen geworven zijn. Echter, net als bij eerder onderzoek onder artsen en maatschappelijk werkers vormden apothekers zich een algemene indruk van studenten op basis van prestaties en gedrag die holistisch van aard waren en niet noodzakelijkerwijs beschreven in de beoogde onderwijseindtermen. Deze resultaten benadrukken dat de bestaande uitdagingen om relevante praktijkgerichte beoordelingen op te nemen in de beoordeling van competenties van zorgprofessionals ook internationaal voor de farmacie gelden.

Hoofdstuk $\mathbf{3}$ gaat dieper in op het onderzoek van deze verwachtingen die supervisoren in de ziekenhuispraktijk hebben van de werkplekprestaties van coassistenten Farmacie. Op basis van de authentieke ervaringen die supervisoren met ons deelden in de interviews uit de in Hoofdstuk 2 beschreven studie ontwikkelden we samengestelde prestatiebeschrijvingen of "vignetten". We gebruikten een multimethodische onderzoeksopzet waarbij de Delphi- 
methode werd toegepast om supervisoren de prestaties uit de vignetten te laten beschrijven, gevolgd door interviews waarmee we meer inzicht wilden verkrijgen in beoordelaars' motivering achter deze oordelen. De theorie van Hofstede over cultuurdimensies diende als kader op basis waarvan we de antwoorden van de deelnemende klinisch docenten uit de twee verschillende contexten (Canada en Qatar) met elkaar vergeleken. Onze verwachting dat de oordelen van beoordelaars onderling van elkaar zouden verschillen vanwege de tegenstrijdige maatschappelijke normen en waarden die in deze landen overheersen, zagen we niet bevestigd in onze analyse. Hoewel we constateerden dat er binnen de landen meningsverschillen bestonden over bepaalde beschrijvingen van studentprestaties werd de consensusdrempel voor de verschillen in supervisorimpressies tussen de landen bij slechts twee van de 16 prestatievignetten bereikt. Ons onderzoek wees niet uit dat de Midden-Oosterse cultuur een merkbare invloed had op de manier waarop de klinisch docenten in Qatar de prestaties van coassistenten beschreven. Supervisoren stimuleerden zelfstandigheid in de patiëntenzorg, hechtten minder waarde aan enkele aspecten van teamharmonie en verwachtten van de coassistenten dat zij op gepaste momenten het gezag van de arts in twijfel trokken. Het was echter waarschijnlijker dat de culturen die de oordelen van beoordelaars beïnvloedden samenhingen met de beroepspraktijk en de organisatieomgeving waarin deze studenten en hun supervisoren waren gesitueerd.

Gezien de aannemelijkheid dat de prestatieverwachtingen beïnvloed werden door een gedeelde professionele cultuur, wat over verschillende geografische settings werd aangetoond, wordt in Hoofdstuk 4 de focus verlegd naar het verkrijgen van inzicht in hoe coassistenten Farmacie vanuit verschillende disciplinaire perspectieven worden beoordeeld. De competenties op het gebied van communicatie en samenwerking die voor de intramurale zorg vereist zijn, worden beschouwd als overwegend interprofessioneel van aard en zodoende wordt aangenomen dat de prestaties van coassistenten zich lenen voor teamfeedback. Hoewel het betrekken van verschillende meningen inderdaad waardevolle inzichten kan opleveren voor de werkplekbeoordeling, kan een dergelijke aanpak tot problemen leiden wanneer dit gebeurt zonder te beseffen wat de achterliggende referentiekaders bij deze meningen zijn. Geleid door een constructivistische-gefundeerdetheoriebenadering namen we semigestructureerde interviews af bij 14 leden van een multidisciplinair team uit de afdelingen acute interne geneeskunde van een groot academisch ziekenhuis in Canada. Het onderzoek wees uit dat teamleden de communicatie en samenwerking van coassistenten Farmacie en van andere coassistenten van buiten hun eigen vakgebied volgens beschreven normen of best practices conceptualiseerden. Echter, in de voorbeelden die ze aanhaalden, beoordeelden de teamleden de prestaties die zij van een coassistent verwachtten blijkbaar op basis van hoe nuttig deze bijdragen waren en niet op basis van de specifieke rollen van communicator en samenwerkingspartner die de betreffende student liet zien. De bewering dat zij de ontwikkeling van interprofessionele competenties door coassistenten op hun klinisch-onderwijsafdeling steunden, werd weliswaar onderuitgehaald door het feit dat zij slechts sporadisch contact hadden met studenten van buiten hun eigen discipline. Incidenteel contact en servicegerichte verwachtingen van coassistenten door teamleden binnen de intramurale zorg geven aan dat een multidisciplinaire inbreng in de werkplekbeoordeling mogelijk op bepaalde punten tekortschiet. 
Tegen Hoofdstuk 5 bereiken we het perspectief van de coassistenten Farmacie zelf en de verwachtingen van hun prestaties op de werkplek. In deze studie stelden we de coassistent centraal om ons te richten op de betekenis die zij toekenden aan hun ervaringen binnen de sociale context van teamgerichte patiëntenzorg. Tijdens het coschap in het ziekenhuis in hun afstudeerjaar hielden de coassistenten Farmacie een schriftelijk dagboek bij waarin zij hun ervaringen met interprofessionele communicatie en samenwerking vastlegden. Van deze aantekeningen, alsmede de transcripten van vervolginterviews, maakten we een reflexieve thematische analyse. Hoewel de genoemde coschapsactiviteiten de meeste rollen van samenwerkingspartner en communicator weerspiegelden zoals beschreven in competentiekaders van de opleiding Farmacie en in interprofessionele competentiekaders, was er vrijwel geen routinematig contact met andere zorgprofessionals; teamontmoetingen waren hoofdzakelijk met artsen. De coassistenten Farmacie waren zelden betrokken bij zorg waarbij zij echt afhankelijk waren van anderen. In plaats daarvan zochten zij meestal patiëntgegevens op of informeerden zij anderen over medicatie. Bij deze interacties werden er bijna nooit conflicten met anderen of conflicten over taken ervaren. Onze resultaten bieden inzicht in hoe coassistenten Farmacie tegen de voor teamzorg vereiste competenties aankijken en hoe zij deze ontwikkelen. Nader onderzoek is noodzakelijk om te begrijpen hoe een dergelijke beperkte mate van interprofessionele communicatie en samenwerking uiteindelijk een nadelige uitwerking zou kunnen hebben op de kwaliteit van de patiëntenzorg.

In Hoofdstuk 6 vatten we de belangrijkste bevindingen uit alle hoofdstukken samen en zetten we op een rij hoe de onderzoeksvragen aan de hand van de zes omgevingspatronen waarvan in klinische leeromgevingen sprake is, werden beantwoord. In de eerste plaats werd niet vastgesteld dat klinisch supervisoren die volgens een gedeeld competentiekader, maar in verschillende nationale culturele contexten studenten Farmacie opleiden de prestaties van studenten op de werkplek verschillend conceptualiseren. Toen we vervolgens onderzochten wat de verwachtingen waren van adequate samenwerking en communicatie door coassistenten over professionele contexten heen, bleken de leden van multidisciplinaire teams latente servicegerichte verwachtingen te hebben. Tot slot strookten de door coassistenten Farmacie vastgelegde praktijkervaringen die overwegend interprofessionele samenwerkings- en communicatierollen weerspiegelden niet met de neiging om hoofdzakelijk informatie over medicatie te verstrekken aan artsen. Er volgt een beschouwing van de algemene implicaties van de verwachtingen die supervisoren, het team en coassistenten hebben van getoonde competenties op de werkplek en van de gevolgen voor toetsing en de eindtermen van het beoogde farmaciecurriculum. Voorbeelden van deze implicaties zijn: hoe cultuur gebruikt wordt om opleidingscontexten te onderzoeken en met elkaar te vergelijken; hoe teams kunnen bijdragen aan het geven van multisource feedback (feedback uit meerdere bronnen); en hoe competentieverwachtingen gebruikt kunnen worden om een curriculum te beschrijven en aan te passen. We presenteren de sterke punten en beperkingen van het werk in dit proefschrift en zetten dwingende lijnen uit voor toekomstig onderzoek. 



\section{Valorisation}

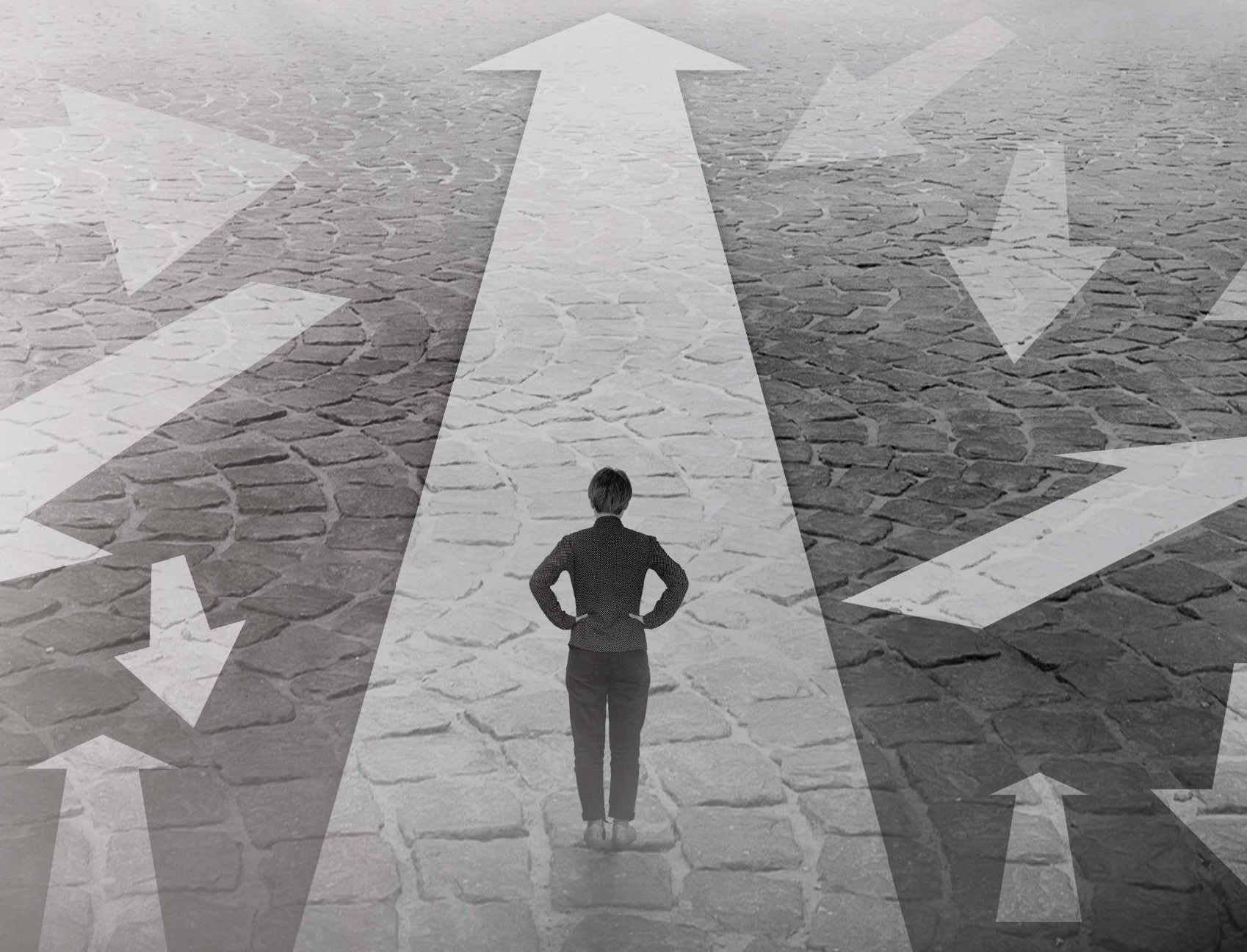




\section{Valorisation}

Pharmacy degree programs have a societal obligation to graduate competent professionals - pharmacists who are able to provide safe and effective care to individual patients and communities. The curriculum is therefore designed for learners to develop and demonstrate specific knowledge, skills, and attitudes (which together may be referred to as "competencies"). To do so, pharmacy education combines classroom instruction with practical training in actual patient care settings, like hospitals, clinics, or community pharmacies. Pharmacy students participate in patient care under the supervision of experienced pharmacists who guide their work, support learning, and judge their performance. The main objective of this research was to enhance understanding of how pharmacist supervisors and other team members in the hospital training workplace form impressions of pharmacy student performance and its impact on their competency development. Our key findings demonstrate how expectations of pharmacy student performance depart from the competencies described in the program curriculum, including exhibited traits and work product oriented to clinical service outputs. We conclude such disconnection between intended and enacted curriculum identified across diverse training contexts may be reconciled through intentional measures, but also merit renewed consideration of the competency standards themselves.

When pharmacy student training moves from classroom campus-based learning to actual patient care settings, pharmacy degree programs rely heavily on pharmacist supervisors to not only foster student integration of theory into practice, but to assess their readiness for independent practice. These results contribute to our evolving understanding of human judgements in the assessment of health professional students and the relationships between expectations and observation/interpretation. Our examination of these through the prism of clinical learning contexts (that is broadly speaking, where and with whom students are training) offers important insight about how pharmacists, other health professionals, and pharmacy students view team communication and collaboration for patient care. Pharmacist trainee shared-care skill development and assessment have implications for contemporary health care models which increasingly draw upon interprofessional member configurations to optimize patient outcomes.

The results of this dissertation are of relevance to broad stakeholder groups. First, workplace-based performance expectations are necessary for students to understand as they shift from learning and practice in controlled classroom settings to assuming the actual patient care responsibilities that accompany clinical training; readiness-for-practice judgements of students practically entail their demonstrating behaviours not always articulated in formal competency descriptions. Second, pharmacist supervisors seeking multisource feedback about pharmacy students joining their teams must appreciate the nature of the underlying performance expectations informing the judgements other team members express. Our findings suggest pharmacist supervisors embed structured opportunities within the busy care environment to promote pharmacy student engagement with other health professionals to optimize competency development for shared patient care. Third, performance expectations and associated competency judgements of pharmacy 
students in workplace-based training are pertinent to program directors and committees responsible for student progress through the curriculum. In a "whole-systems" or programmatic approach to assessment, a holistic view of student performance requires data from various sources - including information from different personnel (people) across learning environments (places) over time. Recognizing contributors' perspectives informs the construction of a representative student performance narrative. Finally, decision-makers setting national pharmacy program competency standards may be advised to integrate descriptions and develop clinical supervisory assessment supports reflecting the needs and conditions of student competency development in contemporary care contexts. Instead of simply stimulating ongoing pursuit of mechanisms to further enforce standardization of workplace-based assessment of the existing competencies, such re-examination may inspire shifts in chosen competencies and their organization.

Findings from these dissertation studies have been shared across broad health professional training, educational research, and healthcare delivery communities internationally. Diverse audiences have accessed our information and ideas thereby creating avenues for stakeholder group engagement for implementation of initiatives and future research. Specific opportunities exist at multiple levels, including locally where I have responsibilities overseeing the undergraduate pharmacy curriculum at one of the largest programs in Canada and as a leader within the academic and institutional networks of interprofessional education and pharmacy practice nationally. Through these conference proceedings, invited addresses, and publications, processes and policies are reconsidered, collaborations are activated, and research programs in pharmacy workplace-based learning energized. 


\section{Dissemination}

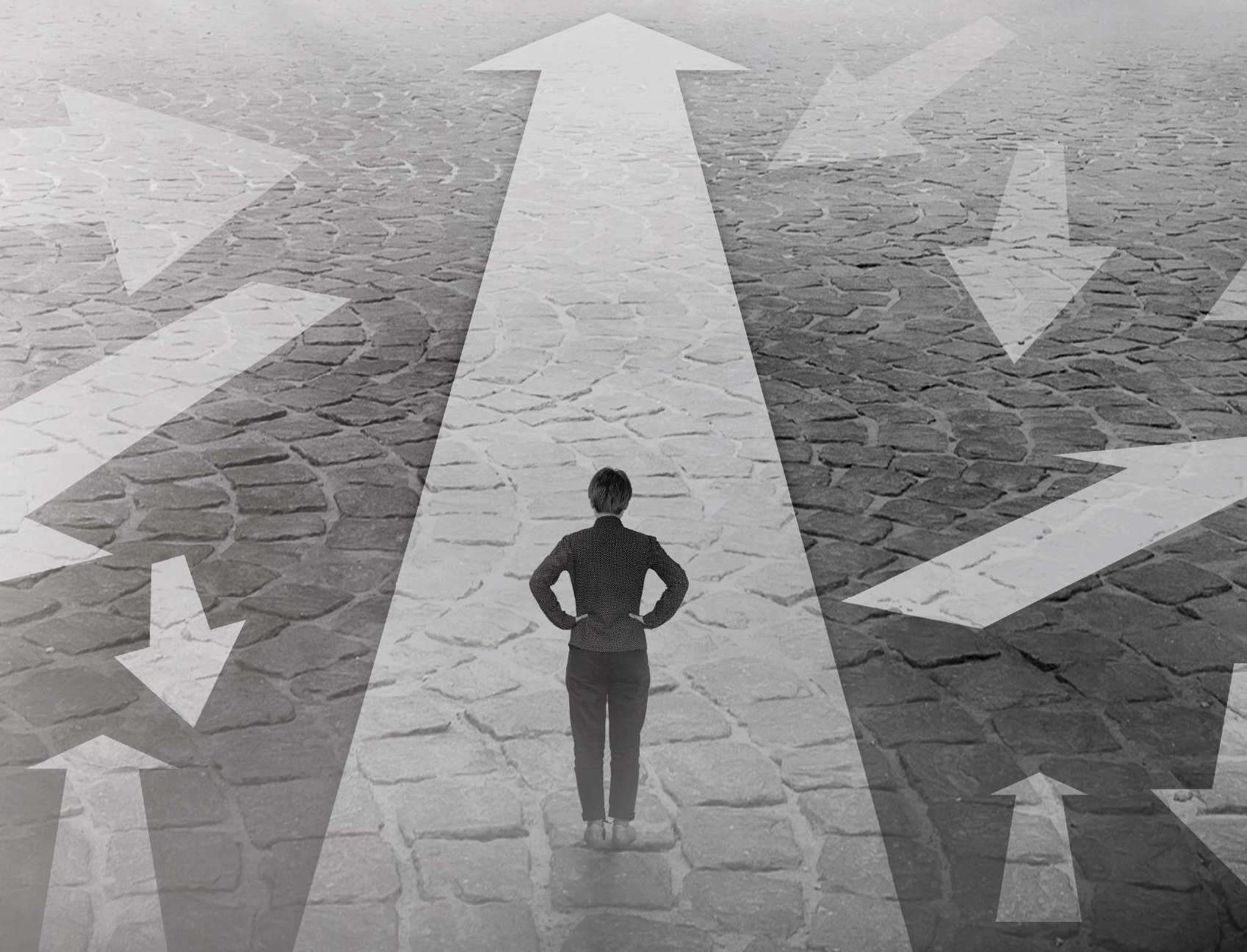




\section{Dissemination}

\section{THESIS DISSERTATION}

Wilbur K, Hassabala N, Saeed OM, Black EK. Describing student performance: a comparison between clinical preceptors across cultural contexts. Med Educ 2017;51:411-22.

Wilbur K, Driessen E, Teunissen PW, Scheele F. Workplace-based assessment in cross-border health professional education. Teach Learn Med 2019; 32:1, 91-103.

Wilbur K, Teunissen PW, Scheele F, Driessen E. Team member expectations of trainee communicator and collaborator competencies - so shines a good deed in a weary world? Med Teach 2021 Jan 21:1-14. DOI: 10.1080/0142159X.2021.1874325.

Wilbur K, Driessen E, Teunissen PW, Scheele F, Yeung J, Pachev G. Pharmacist trainees narrow scope of interprofessional collaboration and communication in hospital practice. Under review.

\section{OTHER THESIS STUDY-RELATED PUBLICATIONS}

Patel M, Agius S. Cross-cultural comparisons of assessment of clinical performance. Med Educ 2017;5: 348-50. Commentary to Thesis Study/Chapter 1

Wilbur K. Progressing from shared care to shared assessment. Acad Med 2019;94:300.

Tong B, St. John M, Li E, Wilbur K. Could interprofessional education assessment in workplace learning be streamlined? J Interprof Educ Pract 2020;19: 100321.

St. John M, Tong B, Li E, Wilbur K. Competency-based education frameworks across Canadian health professions and implications for multisource feedback. J Allied Health 2020;49:e1-11.

Wilbur K, Bensmail N, Ahktar S. Student feedback experiences in a cross-border medical education curriculum. Int J Med Educ 2019; 10:98-105.

Wilbur K, Basha R, Abdelazziz S. Culture effects on experiential training feedback in transplanted Canadian health professional curricula. Int J Med Educ 2017;8:91-8.

\section{PRESENTATIONS}

Invited

Wilbur K. Multisource feedback in competency-based education. Research Rounds. UBC Centre for Health Education Scholarship (CHES), Vancouver, BC (February 19, 2020).

Wilbur K. The Trouble with Categories...in Health Professional Education. UBC Green College Leading Scholars (2018-2020) Lecture Series, Vancouver, BC (November 7, 2019).

* Wilbur K. How does Workplace-Based Assessment 'Work Abroad'? Maastricht University, School of Health Professions Education, SHE Presents New Candidates (November 28, 2017). 
Conference (Oral)

Wilbur K, Pelletier T. Training for collaborative care - what are team expectations of pharmacy students. All Together Better Health $X$ International Conference on Interprofessional Education and Collaborative Practice, Doha Qatar (October 2020) (Accepted Encore, Conference postponed to October 2021 due to COVID).

Wilbur K, Pelletier T. Training for collaborative care - what are team expectations of pharmacy students? AFPC Canadian Pharmacy Education Research Conference, Montreal, Canada (May 2020) (Accepted, Conference cancelled due to COVID).

Wilbur K, Driessen E, Scheele F, Teunissen PW. Multisource feedback of interprofessional competencies: how communication and collaboration are viewed across health disciplines. Canadian Conference on Medical Education (CCME), Vancouver, Canada (April 2020) (Accepted, Conference cancelled due to COVID)

St. John M, Tong B, Li E, Wilbur K. Competency-based education frameworks across Canadian health professions and implications for multisource feedback. Canadian Conference on Medical Education (CCME), Niagara Falls, Canada (April 15, 2019).

Symposia (Oral)

Wilbur K, Yeung J, Pachev G. Interprofessional competency development in clinical learning environments. UBC Centre for Health Education Scholarship (CHES) Celebration of Scholarship, Vancouver, Canada (October 7, 2020).

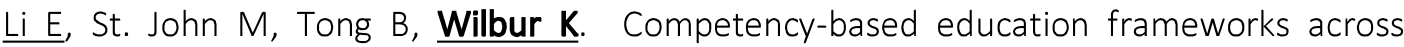
Canadian health professions and implications for multisource feedback. UBC Centre for Health Education Scholarship (CHES) Celebration of Scholarship, Vancouver, Canada (October 2, 2018).

Conference (Poster)

Wilbur K, Pelletier T. Collaborative care - what are multidisciplinary team expectations of hospital pharmacists. Canadian Society of Hospital Pharmacists B.C. Branch Presentation and Competition Vancouver, Canada (virtual, November 5, 2020).

Wilbur K. Describing student performance: a comparison between clinical preceptors across cultural contexts. Maastricht University, School of Health Professions Education (SHE) Academy Conference, Maastricht, the Netherlands (March 27-30, 2017).

Wilbur K, Black EK. Describing student performance: a comparison between clinical preceptors across cultural contexts. $2^{\text {nd }}$ Forum on Advancing Pharmacy Education in the GCC and Middle East $\& 6^{\text {th }}$ Kuwait International Pharmacy Conference, Kuwait City, Kuwait (Encore Poster, February 2017).

Wilbur K, Black EK. Describing student performance: a comparison between clinical preceptors across cultural contexts. Association of Faculties of Pharmacy of Canada Annual Meeting, Richmond, BC (June 2016). 


\section{She Dissertation Series}




\section{SHE Dissertation Series}

The SHE Dissertation Series publishes dissertations of PhD candidates from the School of Health Professions Education (SHE) who defended their PhD theses at Maastricht University. The most recent ones are listed below. For more information go to: https://she.mumc.maastrichtuniversity.nl

Iqbal, Z. (15-12-2020) All stakeholders matter in faculty development: Designing entrustable professional activities for small group facilitation

Tran, QT. (09-12-2020) Nationwide implementation of medical skills training laboratories in a developing country: studies from Vietnam

Pacifico, J. (30-11-2020) Making the Implicit Explicit: Uncovering the Role of the Conceptions of Teaching and Learning and the Perceptions of the Learning Climate in Postgraduate Medical Training.

Nishigori, H. (17-11-2020) Why do doctors work for patients? Medical professionalism in the era of neoliberalism

Oudkerk Pool, A. (06-11-2020) Competency-based portfolio assessment - Unraveling stakeholder perspectives and assessment practices

Geel van, K. (05-11-2020) Lifelong learning in radiology: all eyes on visual expertise

Stammen, L. (16-10-2020) Pursuing - High-Value, Cost-Conscious Care - The Role of Medical Education

Meulen van der, M. (15-10-2020) Assessment of physicians' professional performance using questionnaire-based tools

Matsuyama, Y. (05-10-2020) Contextual attributes fostering self-regulated learning in a teacher-centered culture: learner's professional identity formation is a trigger

Rovers, S. (16-09-2020) Growing knowledge, supporting students' self-regulation in problem-based learning

Bourgeois-Law, G. (03-09-2020) Conceptualizations of remediation for practicing physicians

Giuliani, M. (19-05-2020) A Critical Review of Global Curriculum Development, Content and Implementation in Oncology

Schreurs, S. (20-03-2020) Selection for medical school; the quest for validity

Schumacher, D. (19-03-2020) Resident Sensitive Quality Measures: Defining the Future of Patient-Focused Assessment 
Sehlbach, C. (21-02-2020) To be continued.... Supporting physicians' lifelong learning

Kikukawa, M. (17-12-2019) The situated nature of validity: Exploring the cultural dependency of evaluating clinical teachers in Japan

Kelly, M. (10-12-2019) Body of knowledge. An interpretive inquiry into touch in medical education

Klein, D. (06-11-2019) The performance of medical record review as an instrument for measuring and improving patient safety

Bollen, J. (01-11-2019) Organ donation after euthanasia: medical, legal and ethical considerations

Wagner-Menghin, M. (25-09-2019) Self-regulated learning of history-taking: looking for predictive cues

Wilby, K. (02-07-2019) When numbers become words: Assessors' processing of performance data within OSCES

Szulewski, A. (20-06-2019) Through the eyes of the physician: Expertise development in resuscitation medicine

McGill, D. (29-05-2019) Supervisor competence as an assessor of medical trainees; Evaluating the validity and quality of supervisor assessments

Van Rossum, T. (28-02-2019) Walking the tightrope of training and clinical service; The implementation of time variable medical training 


\section{Acknowledgements}




\section{Acknowledgements}

Pursuit of this doctorate degree has been a privileged experience in all respects.

Given my expatriate status and the opportunities this has afforded, I've toiled on the thesis in over 20 countries across 4 continents in these past 5 years. At cafés, bars, beaches, hotel rooms and pools and lobbies, airport gates and lounges, on airplanes, trains, and ferries. Sometimes alone, sometimes when travel companions were sleeping, showering, working out or even writing up their own research.

This work has been facilitated by the Deans who have welcomed the opportunity to advance educational research skillset among their faculty and my own professional development Doctors Ayman El Kaddi, Mohammad Diab (Qatar University), and Michael Coughtrie (University of British Columbia). Foremost, I am indebted to Dr. Peter Jewesson, my mentor of nearly 25 years, who quite literally altered the trajectory of my life with offers to train and work for him in Vancouver and again in Doha, and whose lessons continue to shape my practice today.

Thank you to my supervisors, Erik Driessen, Fedde Scheele, and Pim Teunissen, who encouraged me throughout this process. I am fortunate to have had their guidance and learn from their broad experiences. Over the years they have made time to Skype with me from their offices (at work and at home), their living rooms, backyards, AMEE congress halls, and recently a COVID/holiday houseboat! As an external candidate, I always felt warmly welcomed on the occasions I travelled to campus and am grateful for the few lucky occasions we have had to meet in Vancouver. In 2015, Jolene Driessen and I joined Maastricht in the same month (me - the university and she- born to her hometown). I am happy to fulfill my promise to Erik to complete this dissertation before she reached high school!

As a graduate student with the School of Health Professional Education I have greatly benefited from its scholarly community and am thankful for the encounters and relationships developed in person and online. These include friendships with other local and international doctoral candidates doing such interesting work and who generously shared their experiences to help sustain my motivation over these years. Perhaps no one more so than my friend and collaborator, Kyle Wilby, whose footsteps I followed into this program and with whom I have spent endless hours (often in aforementioned planes, trains, and lounges) commuting health professional education and practice research ideas into reality.

Occasionally, this thesis was worked on during stays at my childhood home when my mother Betty was cooking supper or my father was mowing the lawn. In the latter half, I've done more writing in a chemotherapy clinic than anyone should. This thesis is dedicated to my selfless and supportive parents and especially my dad, David, who did not live to see its completion. He placed his family and the education of his children at the forefront of everything he did. And so this thesis, like all accomplishments in my life, must be attributed to his unwavering love. 


\section{Curriculum Vitae/Biography}

Dr. Kerry Wilbur (1974, Canada) is a graduate of Canadian baccalaureate (BScPharm, Dalhousie University, 1997), accredited hospital pharmacy residency (QEII Health Sciences Center, Halifax, NS), and doctoral (PharmD, University of British Columbia, 2000) pharmacy programs. Following seven years in clinical practice in internal medicine at the major tertiary care center in Vancouver, B.C., she relocated to the Middle East where she was a founding faculty at the College of Pharmacy at Qatar University (2007). During that time, she served as the first Coordinator of the country's first Continuing Pharmacy Professional Development program (2008-2010), as well as Director of the post-baccalaureate Doctor of Pharmacy Program in (2009-2016) and Associate Dean of Academic Affairs (2016-2017). Dr. Wilbur obtained a Masters in Science in Public Health through the School of Hygiene \& Tropical Medicine, University of London in 2011.

She repatriated in 2017 is now an Associate Professor and Executive Director of Entry-toPractice Education at the Faculty of Pharmaceutical Sciences at the University of British Columbia. The research reported in this dissertation was conducted between 2014 and 2020 in Doha, Qatar (2014-2017) and the province of British Columbia (2017-2020). 